\title{
TYPES OF INTEGRABILITY ON A SUBMANIFOLD AND GENERALIZATIONS OF GORDON'S THEOREM
}

\author{
N. N. NEKHOROSHEV
}

\begin{abstract}
At the beginning of the paper the concept of Liouville integrability is analysed for systems of general form, that is, ones that are not necessarily Hamiltonian. On this simple basis Hamiltonian systems are studied that are integrable only on submanifolds $N$ of the phase space, which is the main subject of the paper. The study is carried out in terms of $k$-dimensional foliations and fibrations defined on $N$ by the Hamiltonian vector fields corresponding to $k$ integrals in involution. These integrals are said to be central and may include the Hamiltonian function of the system. The parallel language of sets of functions is also used, in particular, sets of functions whose common level surfaces are the fibres of fibrations.

Relations between different types of integrability on submanifolds of the phase space are established. The main result of the paper is a generalization of Gordon's theorem stating that in a Hamiltonian system all of whose trajectories are closed the period of the solutions depends only on the value of the Hamiltonian. Our generalization asserts that in the case of the strongest "Hamiltonian" integrability the frequencies of a conditionally periodic motion on the invariant isotropic tori that form a fibration of an integrability submanifold depend only on the values of the central integrals. Under essentially weaker assumptions on the fibration of the submanifold into such tori it is proved that the circular action functions also have the same property. In addition, certain general recipes for finding the integrability submanifolds are given.
\end{abstract}

\section{INTRODUCTION}

The main object of investigation in this paper is autonomous Hamiltonian systems that are not necessarily integrable. Such a system is given by its Hamiltonian function $H$ : $M \rightarrow \mathbf{R}$ defined on a symplectic manifold $M=M^{2 n}$, that is, a manifold equipped with some symplectic structure $\omega^{2}$, where $2 n=\operatorname{dim} M^{2 n}$. Integrable Hamiltonian systems have a relatively simple structure and are fairly well studied, at least in the regular situation. (In the case of "nonregular" integrability there are many interesting questions; see, for example, 9.) But most systems are not integrable on the entire phase space $M$ even in the nonregular sense. What is the structure of the phase flow of nonintegrable systems? It is known that many such systems are integrable, but only on some invariant submanifolds, rather than on the whole of $M$.

First we will look at the regular toric integrability of the system on a submanifold $N \subseteq M$. By this we mean that the $k$-dimensional invariant tori $\Lambda^{b}$ form a locally trivial fibration of this submanifold, so that $N=\bigcup_{b \in B} \Lambda^{b}$, where the parameter $b$ parametrizing

2000 Mathematics Subject Classification. Primary 37J05, 70H12; Secondary 37J15, 37J35, 37J45.

Key words and phrases. Integrable, Hamiltonian, Gordon's theorem, integrability submanifold, conditionally periodic motion, invariant tori, vector field, integrals in involution, symplectic structure, circular action functions, frequency, trajectory, isotropic tori.

This paper was written with partial support of the INTAS grant no. 00-221 and the research was partially carried out during the author's stay at the Littoral University, Laboratory UMR 8101 of CNRS, Dunkerque, France, and at the Milan University, Italy. 
these tori runs over some manifold $B$. The invariance means that the tori $\Lambda^{b}$ are invariant under the action of the local phase flow $\left\{g_{H}^{t}, t \in \mathbf{R}\right\}$ of the system, that is, $g_{H}^{t} \Lambda^{b}=\Lambda^{b}$ for any time $t$. We also assume that the motion of the points of the phase space under the action of this flow is conditionally periodic on the tori $\Lambda^{b}$.

Studying the integrability submanifolds appears to be important for the following reasons. It often turns out that it is such submanifolds $N \subset M$, as well as their neighbourhoods in the whole phase space $M$ of the system, that are of primary interest for applications. In addition, in a neighbourhood of an integrability submanifold the phase flow of the system has the simplest structure. We think that studying the integrability submanifolds is the easiest, but also the most important and immediate step in the study of nonintegrable Hamiltonian systems. In the present paper we are interested only in the behaviour of the system on the submanifold $N$ itself.

There is an extensive literature devoted to finding families of integrable solutions. For example, many examples of this kind of particular solutions to the equations of the dynamics of a rigid body are described in the book [24, which also contains a large list of references related to this problem. Numerous families of integrable solutions were found for the equations of celestial mechanics and many other quite diverse differential equations of importance in applications. In our context the trajectories of all these solutions form integrability submanifolds.

The purpose of our paper is to study general approaches to FINDING the integrability submanifolds, clarifying the CAUSES of existence of such submanifolds, and investigating the QUALITY OF INTEGRABILITY of systems on them, especially, for Hamiltonian systems, and toric integrability. It is here that when we have the highest-quality integrability the generalization of Gordon's theorem for frequencies holds. Note that finding the toric integrability submanifolds interlocks with finding the families of periodic solutions of dynamical systems the unions of whose trajectories can be regarded as a special case of integrability submanifolds; see, in particular, [7, 6].

In fact, the quality of integrability on a given submanifold $N \subseteq M$, as it is understood here, depends on how fully the Hamiltonian character of the system on $N$ is used. One may consider that this character is taken into account most fully in the case of "complete integrability", that is, the integrability of a Hamiltonian system on the entire phase space $M$, which is usually studied. This type of integrability on the whole of $M$ has been studied from general differential-geometric viewpoints in [1, 5, 3, and many other papers.

The case where an integrability submanifold $N \subseteq M$ of the symplectic manifold $M$ is symplectic is easily reduced to the case of integrability on the entire symplectic phase space. The fact is that the restriction of a Hamiltonian system to an invariant symplectic submanifold is also a Hamiltonian system; see Lemma 3.1.1. So if this system with $p$ degrees of freedom, defined on $N=N^{2 p}$, is completely Hamiltonian integrable, its phase space $\left(N^{2 p}, \tilde{\omega}^{2}\right)$, where $\tilde{\omega}^{2}:=\left.\omega^{2}\right|_{N}$, is fibred into the $k$-dimensional invariant isotropic surfaces $\Lambda$; here $k \leq p \leq n$.

If these surfaces are compact sets, then they are diffeomorphic to tori, on which the motion of the system is conditionally periodic. Furthermore, in the case where $N$ is symplectic one can construct normalizing canonical "coordinates" $(I, p, \varphi \bmod 2 \pi, q)$ on $N$ that are generalizations of the action-angle variables $(I, \varphi \bmod 2 \pi)$. In these coordinates the Hamiltonian $\tilde{H}:=\left.H\right|_{N}$ of the system with Hamiltonian $H$ restricted to $N$ has the form $\tilde{H}=\tilde{H}(I)$ (see Proposition 5.1.1); more details about these coordinates can be found in [5]. Hence the frequencies of the conditionally periodic motion on these tori, $\omega_{i}=\dot{\varphi}_{i}=\partial \tilde{H} / \partial I_{i}, i=1, \ldots, k$, depend only on the action variables $I$. This assertion is equivalent to the generalization of Gordon's theorem for frequencies. 
Two simple examples of such a situation are given in $\S 1$. Namely, we produce two systems, both in all probability nonintegrable, that are integrable on certain symplectic submanifolds $N \subset M$. In addition, in $\S 1$ we also illustrate Gordon's theorem itself by the example of the problem of two and many bodies. (The precise statement of the original version of this theorem is given at the beginning of $\S 5.3$.)

Thus, the case where an integrability submanifold $N \subset M$ is symplectic is relatively simple. But the case where $N \subset M$ is a submanifold of general form, for example, of odd dimension, is more complicated. The question arises as to how we can take into account the Hamiltonian characteristics most fully in this case? And under what conditions do the generalizations of Gordon's theorem remain valid? In order to answer these questions we must find the basic types of integrability that a Hamiltonian system can have on a submanifold. In turn, in order to understand this we need to clearly understand the much more simple concept of Liouville integrability for systems of general form, that is, systems that are not necessarily Hamiltonian.

This concept is considered in $\S 2$, which is entirely devoted to systems of general form, which are not considered further in the paper. For such a system with phase space $N$ to be Liouville integrable there must be $k$ vector fields $\left(a_{1}, \ldots, a_{k}\right)=A$ on $N$ that are linearly independent at each point, that are pairwise commutative, and that commute with the vector field $V$ of the given system. (One of the fields in the set $A$ may coincide with the given field $V$, but this is not necessary.) In addition, we require that there exist $r$ functionally independent integrals common to all these $k+1$ vector fields, and that $k+r=\operatorname{dim} N$. The connected components $\Lambda$ of the common level surfaces of these integrals are invariant $k$-dimensional smooth surfaces. They are analogues of the submanifolds $\Lambda=\Lambda^{b}$ considered above, which form a fibration of the integrability submanifold $N$ in the Hamiltonian situation.

In this case we speak about global $A$-integrability of the system of the general form defined by the field $V$. However, if we do not assume that there exist global common integrals, then we speak about local $A$-integrability. This condition is essentially weaker and the whole leaves in $\Lambda$ can have very irregular structure, being very different from their closures, which in the typical situation apparently causes the trajectories of the system that lie on them to be irregular. Therefore global integrability should be regarded as "real" integrability. But local integrability is a necessary condition for global integrability and is often more convenient to study, so it is important.

If the field $V$ is globally integrable on $N$ and in addition the surfaces $\Lambda$ that form a fibration of $N$ are compact sets, then we say that $V$ is torically integrable on $N$. Indeed, in this case each submanifold $\Lambda$ is a torus with conditionally periodic motion on it. Moreover, in a neighbourhood of such a torus one can introduce normalizing "coordinates" $(I, \varphi \bmod 2 \pi)$ in which the original system of general form takes the form $\dot{I}=0, \dot{\varphi}=\omega(I)$, where $I=\left(I_{1}, \ldots, I_{r}\right)$ and $\varphi=\left(\varphi_{1}, \ldots, \varphi_{k}\right)$; see Proposition 2.2.1.

These simple facts given in the first half of $\S 2$ enable us to understand the various types of integrability that a Hamiltonian system defined on a symplectic manifold $M$ has on an arbitrary invariant submanifold $N \subseteq M$ and to clarify the connections between these types, which we discuss in $\S 3$. In particular, apart from the division into local, global, and toric integrability, we can distinguish general integrability (that is, as in the general non-Hamiltonian situation of $\S 2$ ), weakly Hamiltonian, and (strongly) Hamiltonian integrability. We describe briefly the last two types of integrability, which are of higher quality and take into account the Hamiltonian characteristics, here confining ourselves to the local case; see the precise definitions in $\S 3$.

Weak Hamiltonian integrability on $N \subseteq M$ takes place in the following situation. In addition to the original system with Hamiltonian $H$, whose vector field we denote 
by $J d H$, on $M$ there are $k$ Hamiltonian systems with Hamiltonians $\left(Z_{1}, \ldots, Z_{k}\right)=Z$ which have the following properties. The vector fields $J d H, J d Z_{1}, \ldots, J d Z_{k}$ are tangent to $N$ and are pairwise commutative on $N$. It is also required that all the leaves $\Lambda \subseteq N$ defined by the $k$ fields $J d Z_{1}, \ldots, J d Z_{k}$ be isotropic $k$-dimensional submanifolds of $M$. The leaves $\Lambda$ being isotropic is equivalent to the fact that the Hamiltonians $Z_{1}, \ldots, Z_{k}$, $Z_{i}: M \rightarrow \mathbf{R}$, of these $k$ Hamiltonian fields are pairwise in involution on $N$, which we denote by $\left.\{Z, Z\}\right|_{N}=0$. It is also assumed that at each point $m \in N$ the differential of the Hamiltonian function $H$ of the system is a linear combination of the differentials of the functions in the set $Z=\left(Z_{1}, \ldots, Z_{k}\right)$. The last condition is called the sliding condition.

In this case $Z$ is called a set of "weakly Hamiltonian-sliding symmetries" of the system on $N \subseteq M$, and the system itself is said to be "locally weakly Hamiltonian- $Z$-integrable" on $N$; see precise formulations in Definition 3.2.4. It is easy to show that the restriction of such a field $J d H$ to $N,\left.J d H\right|_{N}$, is $\tilde{A}$-integrable in the sense of systems of general form considered in $\S 2$, where $\tilde{A}=\left(\left.J d Z_{1}\right|_{N}, \ldots,\left.J d Z_{k}\right|_{N}\right)$.

Among "weakly integrable" systems we distinguish the "strongly integrable" ones, which we call "locally Hamiltonian- $Z$-integrable" on $N$; see Definition 3.2.4. These are systems such that the submanifold $N$ and the set $Z$ have one more property: "internal regularity" of $Z$ on $N$. This property consists in that the set $\tilde{Z}=\left(\tilde{Z}_{1}, \ldots, \tilde{Z}_{k}\right)$ of the restrictions $\tilde{Z}_{i}:=\left.Z_{i}\right|_{N}$ of these functions to $N$ is regular in the sense that the differentials of these restricted functions are linearly independent at each point $m \in N$. This means that, in contrast to the "weak" case, not only are the differentials of the functions in the set $Z$ linearly independent at every point $m \in N$, but also so are the functions in the set $\tilde{Z}$, which is a stronger condition. It is easy to show that in the "weak" situation this condition that $Z$ has "internal regularity" on $N$ is automatically satisfied if $N \subseteq M$ is a symplectic submanifold, so that this case is, in fact, a "strong" one; see Corollary 3.3.1.

One of the main objectives of the paper is the study of arbitrary integrability submanifolds, that is, ones that are not necessarily symplectic. The investigations carried out in $\S \S 3-6$ show that if a system is strongly Hamiltonian-integrable on such a submanifold, and even globally, then its restriction to this submanifold $N$ has virtually the same properties as the integrable system that is more often studied, that is, a completely integrable Hamiltonian system on a symplectic manifold. In particular, in the case where the fibres $\Lambda \subset N$ are compact sets all the generalizations of Gordon's theorem are valid on the surface $N$. If in addition $N \subset M$ is symplectic, then the system has exactly the same properties as completely integrable Hamiltonian systems. So we may suppose that the fundamental role in ensuring high-quality, Hamiltonian integrability on any submanifold $N$ is played by the condition that $\tilde{Z}$ is regular on $N$.

Moreover, this conclusion is supported by the following two facts. First, it is actually required to ensure that the system in the situation of the Liouville-Arnol'd theorem and its generalizations is completely integrable; see [1, 5. Here it has the form of the map $Z$ : $M \rightarrow \mathbf{R}^{k}$ defined by the set $Z$ of "central" integrals being regular. Second, the condition of internal regularity of $Z$ on $N$ does not automatically follow from the other conditions of strong integrability and does not even follow from weak integrability. Indeed, an example given in $\S 5.4$ shows that there exist systems that are weakly Hamiltonian- $Z$-integrable on a nonsymplectic submanifold $N$, but $Z$ is not internally regular on $N$. (The same example is a counterexample to the generalization of Gordon's theorem for frequencies to a submanifold.)

Actually, in $\S 3$, apart from sets $Z=\left(Z_{1}, \ldots, Z_{k}\right)$ of strongly and weakly Hamiltoniansliding symmetries on $N$, we consider sets $Z$ of a similar type satisfying essentially weaker conditions, namely, sets of "pseudo-Hamiltonian-sliding symmetries". This means 
that in the "weak" definition, instead of the condition that the Hamiltonian vector fields $J d H, J d Z_{1}, \ldots, J d Z_{k}$ be pairwise commutative, we only require that the $k$ fields $J d Z_{1}, \ldots, J d Z_{k}$ define a foliation of the submanifold $N \subseteq M$ into isotropic leaves and that each of these fields commutes with the field $J d H$; see Definition 3.2.4.

Thus, if in addition a locally Hamiltonian- $Z$-pseudo-integrable system (that is, a system with pseudo-Hamiltonian sliding symmetries $Z$ ) is $\tilde{A}$-integrable in the sense of systems of general form, where, as above, $\tilde{A}=\left.J d Z\right|_{N}$, then $Z$ is a set of weakly Hamiltoniansliding symmetries on $N$. But, instead of requiring that the fields $J d Z_{1}, \ldots, J d Z_{k}$ are pairwise commutative, in the pseudo-Hamiltonian situation we can require that $\tilde{Z}$ is regular, and then this commutativity condition will be automatically satisfied (see Proposition 3.4.1), which seems amazing at first glance. In this case we even have strongly Hamiltonian integrability.

The study of integrability in $\S 3$ is carried out mainly on the basis of differential geometry, which is used to study the foliations and fibrations defined by the fields $J d Z_{1}, \ldots, J d Z_{k}$ on $N$. To foliations there corresponds local integrability, and to fibrations, global. We put more emphasis on studying local integrability, that is, merely foliations. But we also consider fibrations, that is, the case where the leaves $\Lambda \subseteq N$ of these foliations are globally "gripped" by common invariants, and the case where in addition the $\Lambda$ are tori. In these two cases systems with sliding symmetries $Z$ on $N$ are called (globally) Hamiltonian- $Z$-integrable and torically Hamiltonian- $Z$-integrable on $N$, respectively. Taking into account the separate division of integrability into types with respect to the degree of Hamiltonian integrability considered above we obtain quite a lot of kinds of integrability; see the diagram:

$$
\left[\begin{array}{cc}
\text { foliations } & \begin{array}{c}
\text { locally } \\
\text { fibrations }
\end{array} \\
\begin{array}{c}
\text { (globally) } \\
\text { torically }
\end{array}
\end{array}\right] \otimes\left[\begin{array}{c}
\text { pseudo } \\
\text { weakly } \\
\text { (strongly) }
\end{array}\right] \begin{aligned}
& \text { Hamiltonian } \\
& \text { integrability }
\end{aligned}\left[\begin{array}{c}
\text { of a system } \\
\text { of a set of functions }
\end{array}\right] .
$$

The main connections between these cases are established in Propositions 3.3.1-3.3.4 and 3.4.1 and their corollaries. Some of them were discussed above.

In the case of systems of general form, that is, ones that are not necessarily Hamiltonian, the second column describing the quality of Hamiltonian integrability should be removed, as well as the following word "Hamiltonian", leaving just "integrability". In addition, in the last column in the lower row "of a set of functions" should be replaced by "of a set of vector fields". Actually, Hamiltonian-pseudo-integrable systems are not even integrable in this ordinary sense; that is, as systems of general form, they are merely potentially Hamiltonian-integrable, which was discussed above. The last column appeared because it is often convenient to abstract oneself from the system and consider sets of functions that either themselves, or via the corresponding vector fields, define a foliation or fibration of a submanifold of the symplectic manifold of the required form. The situation is similar also in the case of systems of the general form.

In $\S 4$ we only study systems that are globally integrable on submanifolds $N \subseteq M$, and only in the strongest sense, that is, they are Hamiltonian. Moreover, the study is not based on fibrations, but is carried out in the spirit of the original versions of the Liouville-Arnol'd theorem, which often turns out to be more convenient for applications. More precisely, the definitions of this integrability are stated in terms of sets of functions, namely, in terms of sets $(Z, \mathcal{F})$ that are "Hamiltonian-integrable" on $N$; see Definition 4.2.1. Here, as above, $Z=\left(Z_{1}, \ldots, Z_{k}\right)$ is a set of functions on the whole of $M$ such that $\left.\{Z, Z\}\right|_{N}=0$, that is, $d Z_{j}\left(J d Z_{i}\right)=0$ on $N$, where $i, j=1, \ldots, k$, and $N$ is assumed to be invariant under the action of the flows of all the systems with Hamiltonians $Z_{i}$. These functions are called central functions in the whole set $(Z, \mathcal{F})$. The subset $\mathcal{F}$ consists of $l$ functions on $N$ such that $d \mathcal{F}_{s}\left(J d Z_{i}\right)=0, s=1, \ldots, l$. 
We also assume that $2 k+l=\operatorname{dim} N$ and that the functions in the set $(\tilde{Z}, \mathcal{F})$ "associated" with $(Z, \mathcal{F})$, where $\tilde{Z}:=\left.Z\right|_{N}$, define a regular map $N \rightarrow \mathbf{R}^{k+l} ;$ cf. [5. Thus, $(\tilde{Z}, \mathcal{F})$ is a "maximal" set of integrals on $N$ common to the fields $J d Z_{1}, \ldots, J d Z_{k}$. The existence of such a set $\mathcal{F}$ turns the $k$-dimensional foliation defined by these fields on $N$ into a fibration. Here, if the connected components $\Lambda \subseteq N$ of the common level surfaces of the functions in the set $(\tilde{Z}, \mathcal{F})$ are compact, then these components are $k$-dimensional tori.

We remark that the conditions we make here of (strong) Hamiltonian integrability of a system on $N \subset M$ (the system itself is not taken into account) are an immediate generalization of the conditions in the main theorem on lower-dimensional integrability on the entire phase space $M$ in [5] stated in $\S 1$ there. We emphasize that this is a generalization to the case of an arbitrary, not necessarily symplectic, submanifold $N$.

By a system being $(Z, \mathcal{F})$-integrable on $N$ we mean a system with Hamiltonian $H$ for which the $Z$-sliding condition holds on $N$, that is,

$$
d H=\sum_{i=1}^{k} \lambda_{i} d Z_{i}
$$

where $\lambda_{i}=\lambda_{i}(m), m \in N$. In the case where the fibres $\Lambda$ are compact they are $k$ dimensional tori, and the motion on them under the action of the phase flow of the system is conditionally periodic. In $\S 4$ we also state Propositions 4.3.1 A) and B) on the equivalence of the definitions of $\S 4$ and the corresponding definitions of $\S 3$, which are more invariant and geometric.

The main contents of $\S 5$ are the statements of the generalizations of Gordon's theorem for action variables (Theorem 5.5.1 A)) and for frequencies (Theorem 5.5.1 B)) to the case of systems torically integrable on a submanifold. In more detail, the contents of $\S 5$ are as follows. At the beginning we state Proposition 5.1.1 on the existence of generalized action-angle variables $(I, p, \varphi \bmod 2 \pi, q)$ on a symplectic submanifold $N \subseteq M$. Then we describe how to construct a set $I=\left(I_{1}, \ldots, I_{k}\right)$ of circular action functions in quite a general situation when the submanifold $N$ has a locally trivial fibration into isotropic tori, which is not connected with any vector fields in any way.

The second half of $\S 5$ contains the statements of the two main results of the paper, the aforementioned Theorems 5.5.1 A) and B). They can be regarded as generalizations of Gordon's theorem (see [11, 12]), which asserts the following. Suppose that all the solutions of an (autonomous) Hamiltonian system are periodic; then the period $T$, the frequency of solutions $\omega$, and the circular action function $I$ depend only on the value of the Hamiltonian function $H$ on the trajectory of a given solution. For example, in the problem of two bodies attracting each other according to Newton's law all the solutions with negative energy and nonzero angular momentum are periodic, and the period of these solutions and their action depend only on the total energy, which is equal to the Hamiltonian function $H$.

In Theorem 5.5.1 A) we consider separately the fibration of an arbitrary submanifold $N \subseteq M$ into the isotropic tori defined by the vector fields $J d Z_{1}, \ldots, J d Z_{k}$ that are not necessarily pairwise commutative on $N$; in other words, a pseudo-Hamiltonian toric fibration. We show, in fact, that the action functions $I_{i}, i=1, \ldots, k$, are locally constant on the intersections of the common level surfaces $Z^{-1}(z)$ of the functions in the set $Z$ with $N$.

Theorem 5.5.1B) asserts that if a system is Hamiltonian-torically $Z$-integrable on $N$, then the frequencies $\left(\omega_{1}, \ldots, \omega_{k}\right)=\omega$ of the conditionally periodic motion on the invariant tori, which are the fibres of the corresponding fibration of $N$, depend locally only on the functions in the set $Z$ defining this fibration: $\omega=\omega(Z)$. Or, in terms 
of the sets of functions in $\S 4$, they depend only on the central functions $Z_{1}, \ldots, Z_{k}$ in the set $(Z, \mathcal{F})$. Thus, the number of parameters on which the frequencies depend is at most the dimension $k$ of the invariant tori, while the codimension of these tori in the submanifold $N$ whose fibration they form can be arbitrarily large. Note that in the case of Hamiltonian integrability this codimension is at least $k$ because $\tilde{Z}$ is assumed to be regular.

We also show that under the hypotheses of this theorem, $H, I$, and $\omega$ satisfy the same relations as they do when $N$ is symplectic, that is, $\omega=\frac{\partial H}{\partial I}(I)$. We emphasize that the condition that $N$ can be fibred into isotropic tori in Theorem B) is essentially stronger than in Theorem A).

Immediately before the statements of these theorems, in $\S 5.4$ we give the counterexample described above showing that Gordon's theorem for the frequencies of periodic solutions cannot be generalized to a submanifold. In this example we consider a submanifold $N \subseteq M$ that consists entirely of the closed trajectories $\Lambda$ of a certain system and lies on the level surface $H^{-1}(0)$ of the Hamiltonian function $H$ of this system. Thus, the Hamiltonian $H$ is also the only central function, that is, the only function in $Z$, but the period of the solutions whose trajectories lie on $N$ is not constant. In fact, this does not contradict Theorem B), since one of the conditions of Hamiltonian integrability is not satisfied, namely, the condition of regularity of the restriction $\left.H\right|_{N}$ of the function $H$ to $N$.

In $\S 6$ we prove all the assertions that were stated in $\S \S 3-5$ but not proved there because their proofs are very detailed.

Finally, the second half of $\S 2$, namely $\S 2.3$, and the whole of $\S 7$ are devoted to the problem of finding the integrability submanifolds: in $\S 2$ we look at systems of general form, and in $\S 7$, Hamiltonian systems. The symmetries which we have considered so far are abelian, and only such symmetries can guarantee integrability. At the beginning of $\S 2.3$ we give an argument showing that if we have a nonabelian local Lie group of symmetries, the sliding condition specifies the maximal submanifold $N \subset M$ of sliding symmetries, that is, an invariant submanifold on which the vector field of the system is tangent to the group orbits. Then under certain conditions, which hold, for example, for many compact symmetry groups, we prove the toric integrability of the system on such an $N$. Thus nonabelian symmetry groups are kind of transformed into compact abelian ones.

In $\S 7$ we remind the reader, using terms that suit our aims, how given a Poisson action of a local nonabelian Lie group on the entire phase space $M$, we can construct a "centred" set of functions $(Z, \mathcal{F})$, that is, such that $\{Z, Z\}=0$ and $\{Z, \mathcal{F}\}=0$ on the whole of $M$; more precisely, see Definition 4.1.2. In the case $N=M$ such sets of functions coincide with the sets $(Z, \mathcal{F})$ discussed earlier that are Hamiltonian-integrable on $N \subseteq M$. The same is also done in the more general situation of a Poisson closed set of functions $F$. We also show that if the functions in such a set $F$ are integrals of the system, then the sliding condition specifies the maximal $Z$-integrability submanifold. Here $Z$ is a "central" subset of a centred set of functions $(Z, \mathcal{F})$, which is constructed from the set $F$.

In addition, in $\S 7$ we consider the case where $F$ is not a set of integrals on the whole of $M$. In this situation, specifying the integrability submanifolds $N$ cannot be achieved using just the sliding condition and is much more difficult. This is because the sliding condition now no longer has to specify an invariant submanifold. The submanifolds $N$ obtained here are called "equilibrium submanifolds". The phase curves lying on $N$ generalize relative equilibrium positions (see, for example, [3]), which exist only if there 
is a reduction of the system. We also consider certain other more general situations, and methods for finding the $Z$-integrability submanifolds in these situations.

The main results here concerning the generalizations of Gordon's theorem were stated in [10, and their proofs were also outlined there. These proofs are given in full in the present paper. Note that Gordon's theorem [12] was published practically at the same time when the studies on lower-dimensional integrability appeared [4, 5]. Both Gordon's theorem and the generalizations of it to the case of a "Hamiltonian" fibration of the entire phase space $M$ into invariant isotropic tori of any dimension follow from those studies as obvious corollaries; see $\S 1$ in 5 . Far from being exhausted by the generalizations of Gordon's theorem this section in [5] contains a more difficult result-normalizing canonical "coordinates" were constructed: the generalized action-angle coordinates mentioned earlier. But the author knew nothing about the paper [12] at that time and learned about Gordon's theorem much later from the book [11.

The present paper can be regarded as a continuation of our work on "lower-dimensional" or "isotropic" integrability of Hamiltonian systems started in [4, 5]. In these papers we studied the completely integrable case, where the entire phase space of the system is fibred into invariant isotropic tori of any dimension, rather than necessarily into Lagrangian tori as was done in the original version of the Liouville-Arnol'd theorem; see [1]. Recall that Lagrangian surfaces have the maximum dimension among the isotropic surfaces. In the present paper we study integrability on submanifolds of the phase space started in [7] in the spirit of the Liouville-Arnol'd theorem; see also [6.

Many facts, including those given in this paper, show that in the study of integrability on submanifolds new effects appear, which do not exist in completely integrable systems. Therefore we can speak about the theory of integrability on submanifolds of phase space, which is separate from ordinary integrability theory. We plan to continue the study of integrability on submanifolds, and this paper is supposed to lay the basis for this. We give a lot of attention to fundamental notions and assertions.

The author is grateful to E. Kudryavtseva, S. Shadrin, as well as to B. Zhilinskiu and D. Sadovskiı for useful discussions and remarks.

\section{EXAMPLES OF INTEGRABILITY SUBMANIFOLDS AND AN ILLUSTRATION OF GORDON'S THEOREM}

This section is devoted exclusively to the study of Hamiltonian systems. In $\S 1.1$ we give two heterogeneous concrete examples of Hamiltonian systems that are integrable on certain submanifolds $N$ of the phase space $M$ but, in all probability, are nonintegrable on the whole of $M$. The same simple examples are used to elucidate the role of "centred" sets $\Phi=(Z, \mathcal{F})$ of functions defined on the whole of $M$ and the special role of their central subsets $Z$; see Definition 4.1.2. In $\S 1.2$ Gordon's theorem is illustrated by the two-body problem, as well as the $n$-body problem. It is explained how in simple cases this theorem can be obtained from the flows defined by integrals of the system by means of "spreading".

1.1. Examples. It may seem that the number of integrability submanifolds in concrete systems of interest in applications will be small and that all such submanifolds can be relatively easily found. The following example shows that this is far from being the case and that there can be infinitely many such submanifolds. Moreover, the closure of the union of integrability submanifolds can coincide with the entire phase space.

Example 1. Consider the Hamiltonian system corresponding to the geodesic flow on a Riemannian compact manifold with an arbitrary Riemannian metric whose curvature is everywhere negative. It is well known that there are infinitely many isolated closed 
geodesics on such a manifold. Moreover, the union of all the closed phase curves of the geodesic flow, that is, of the liftings of the closed geodesics to the phase space $M$ of the system is everywhere dense in $M$. This means that there exist 2-dimensional integrability submanifolds $N=N_{\kappa}^{2}$ such that the closure of their union $\bigcup_{\kappa} N_{\kappa}^{2}$ coincides with the entire phase space: $\overline{\bigcup_{\kappa} N_{\kappa}^{2}}=M$. Here $\kappa$ is some nonregular discrete parameter.

The families of periodic trajectories described above may be called "wild" because their existence does not follow from any obvious symmetries of the vector field in the phase space of the system. (Such families in a way resemble wild Lie groups, which also do not conform to certain frameworks.) In all probability, as a rule, the fields considered in Example 1 may not only have no geometrically obvious symmetries, but may have no symmetries at all, apart from this field itself. These vector fields are independent of time $t$, that is, the system is autonomous, but this implies only the existence of the first integral of the system - the Hamiltonian function $H$.

We also remark that, apart from a few exceptions, all the periodic solutions in Example 1 have large period and therefore are not interesting from the viewpoint of direct applications. On the contrary, many trajectories of the "relative equilibrium position" type (see [3]) are interesting from this viewpoint. We give a simple example of such trajectories.

Example 2. Consider the problem of $s$ bodies attracting each other according to Newton's law. We assume that the centre of mass of all the bodies is stationary; then the position and velocity of one body are determined by the positions and velocities of the other bodies. Consequently, $\operatorname{dim} M=6(s-1)$, where $M$ is the phase space of the system, which coincides with the cotangent bundle $T^{*} \mathcal{E}^{3(s-1)}$ of the configuration space $\mathcal{E}^{3(s-1)}=\mathbf{E}^{3(s-1)} \backslash \Sigma$. Here $\mathbf{E}^{3(s-1)}$ is Euclidean $3(s-1)$-dimensional space, and $\Sigma$ the subset corresponding to collisions of the bodies. Apart from the energy integral $H$, the system has three additional integrals $\mathcal{M}_{1}, \mathcal{M}_{2}, \mathcal{M}_{3}$ - the components of the vector $\overrightarrow{\mathcal{M}}$ giving the total angular momentum of the bodies. All the trajectories of the vector field defined by the Hamiltonian function $\mathcal{M}=\sqrt{\mathcal{M}_{1}^{2}+\mathcal{M}_{2}^{2}+\mathcal{M}_{3}^{2}}$ for $\mathcal{M} \neq 0$ are closed and form a locally trivial fibration of the entire phase space $M=T^{*} \mathcal{E}^{3(s-1)}$, with the exception of the subset of points where $\mathcal{M}=0$.

The equation $d H(m)=\lambda(m) d \mathcal{M}(m), \quad \lambda(m) \in \mathbf{R}$, imposed on the points $m \in M$ for $\mathcal{M}(m) \neq 0$ specifies a 4-dimensional symplectic submanifold $N=N^{4} \subset M$. The submanifold $N^{4}$ consists of the trajectories of solutions, called circular periodic solutions of Lagrange, that correspond to the circular rotations of all the bodies of the system around the common centre of mass with the same angular velocity. Then the bodies of the system are situated in a special way; for example, for $s=3$ they are at the vertices of an equilateral triangle. According to the generalization of Gordon's theorem the period of such rotations depends only on the total energy $H$ of the system.

Note that in the first of these two examples integrability was determined by the Hamiltonian $H$ itself, and in the second by the set $\left(\mathcal{M}, \mathcal{M}_{1}, \mathcal{M}_{2}\right)$. In other words, it is these sets of functions whose common level surfaces specify the closed phase curves of the system on the integrability submanifolds $N$. In the general case such a set of functions on $M$ has the form of a regular set $\Phi=(Z, \mathcal{F})$, where $Z=\left(Z_{1}, \ldots, Z_{k}\right)$ and $\mathcal{F}=\left(\mathcal{F}_{1}, \ldots, \mathcal{F}_{l}\right)$, such that on $M$ all $k$ functions in the subset $Z$ are in involution with all $k+l$ functions in the set $(Z, \mathcal{F})$. A set $(Z, \mathcal{F})$ with these properties is called centred, and the functions in the subset $Z$ central; see the definitions in $\S 4.1$.

Suppose that $N \subseteq M$ is some submanifold invariant under the flows of the system with Hamiltonians $Z_{i}, i=1, \ldots, k$, and such that $2 k+l=\operatorname{dim} N$ and the map $\left.\Phi\right|_{N}: N \rightarrow \mathbf{R}^{k+l}$ is regular; cf. [5. (In this situation the sets $\Phi=(Z, \mathcal{F})$ differ from the sets $(Z, \mathcal{F})$ we looked at in the Introduction when we discussed $\S 4$ (see also Definition 4.2.1), only in 
that the functions in the subset $\mathcal{F}$ are defined on the whole of $M$, rather than merely on $N$.) Then the sets $\Phi$ define by their common surfaces a fibration of $N$ into fibres $\Lambda$ that are the integral surfaces of the foliation defined by the vector fields $J d Z_{1}, \ldots, J d Z_{k}$. When the system is $\Phi$-integrable on $N$, that is, when the sliding condition $d H(m)=$ $\sum \lambda_{i}(m) d Z_{i}(m)$ holds on $N$, the frequencies in the generalized Gordon's theorem will depend on the central functions in the set $\Phi$. In the examples considered above we have $k=1$ in both cases, while $l=0$ and 2 , respectively.

It is natural to ask when do the centred sets $(Z, \mathcal{F})$ appear? Poisson actions of local Lie groups on symplectic manifolds are one of the main and simplest sources of them; see [3]. The two examples given above are special cases of this situation. Indeed, in the first example we may assume that the existence of such a set of functions $(Z, \mathcal{F})$ on $M$ is connected by the Noether duality with the action on $M$ of the local symmetry group $\mathbf{R}$ coinciding with the phase flow of the system itself. In the second example it is connected in the same way with the action of the compact Lie group $G=S O(3)$ of orthogonal transformations of 3-dimensional space. The group $S O(3)$ acts in the obvious way in "physical" Euclidean space $\mathbf{E}^{3}$ and induces an action on the configuration space $\mathcal{E}^{3(s-1)}$, and thus induces an action also on the phase space of the system $M^{6(s-1)}=$ $T^{*} \mathcal{E}^{3(s-1)}$. According to the Noether duality this action defines the set of integrals $\left(\mathcal{M}_{1}, \mathcal{M}_{2}, \mathcal{M}_{3}\right)$, which can be replaced by the almost everywhere equivalent and regular centred set $\left(\mathcal{M}, \mathcal{M}_{1}, \mathcal{M}_{2}\right)$.

In fact, the existence of a Poisson action of any local Lie group $G$ on a symplectic manifold gives rise by the Noether duality to a set $(Z, \mathcal{F})$ with these properties. In $\S 7$ we remind the reader of the process of constructing such a set in the general case (see [1, [3, 16, 23]) and discuss some methods of finding the integrability submanifolds $N$, in particular, by specifying $N$ in $M$ by the aforementioned "sliding" condition. This simple but effective method is a generalization of the method used for specifying $N$ in Example 2 . As a whole, $\S 7$ is to some extent a continuation of this subsection.

1.2. The two-body problem as an illustration of Gordon's theorem. This completely integrable system is a classical example of the application of Gordon's theorem; it is also the simplest. The system has 3 degrees of freedom, and all its trajectories with negative energy $H<0$, apart from the exceptional ones, are closed. The five-parameter family of closed trajectories forms a fibration of the corresponding domain of the phase space $M^{6}$, and their 4-parameter subfamilies form fibrations of the level surfaces $H^{-1}(h)$ of the Hamiltonian function $H$ for $h<0$. On each such surface the period of motion along the trajectories lying on them is the same, which at first glance seems to be quite strange and amazing.

Indeed, as already mentioned, on $M^{6}$ there is a Poisson action of the group $S O(3)$ preserving the Hamiltonian function, but the orbits of its action are 3-dimensional, while the number of parameters of the closed trajectories on $H^{-1}(h)$ is equal to 4 . Of course, there is also the action of the system's phase flow itself, but it does not spread the trajectories, since its orbits are these trajectories themselves.

The explanation is in the fact that, apart from $H$ and the integrals $\mathcal{M}_{1}, \mathcal{M}_{2}, \mathcal{M}_{3}$ corresponding to the action of the group $S O(3)$, the system also has the fifth integral, whose existence actually causes the trajectories of the system in the 6-dimensional phase space to be closed. The phase flow of this integral together with the flows of the integrals $\mathcal{M}_{1}, \mathcal{M}_{2}, \mathcal{M}_{3}$ spread one closed trajectory over the entire level surface $H^{-1}(h)$. These flows preserve $H$ and are symplectomorphisms and therefore preserve both the property that the trajectories are closed and the period, which explains Gordon's theorem in this case.

Using this argument one can prove the weak variant of Gordon's theorem given in the 
Introduction, that is, with no elaboration on the relations between $\omega, H$, and $I$. This deals with systems whose phase space is fibred into closed trajectories. This method can also be used to obtain this weak variant when the entire phase space has a Hamiltonian fibration into invariant isotropic tori of any dimension. However, if we have toric integrability on a nonsymplectic submanifold, then such simple considerations alone do not suffice, if only because the "noncentral" integrals can be defined only on an integrability submanifold $N$. (In the example considered above the noncentral integrals are $\mathcal{M}_{1}, \mathcal{M}_{2}, \mathcal{M}_{3}$ and the fifth integral, and the only central one is the Hamiltonian $H$, so that $k=1$ and $l=4$.) The fact that this situation does occur (apparently quite often) is shown by the system given in $\S 5.4$ as a counterexample to Gordon's theorem. Such integrals do not define vector fields, and this does not allow one to spread the invariant tori.

Arguments based on "spreading" also have the disadvantage that they do not allow one to establish the equalities $\omega_{i}=\frac{\partial \tilde{H}}{\partial I_{i}}(I), i=1, \ldots, k$, for the frequencies $\omega_{i}$ of the conditionally periodic motion and thus obtain the generalization of the strong variant of Gordon's theorem. These relations between $\omega, H$, and $I$ are obtained on the basis of the generalized action-angle variables normalizing the Hamiltonian $H$; see [5].

Note that when the number of bodies of the system is greater than two, $s>2$, there exist periodic solutions that are analogues of the periodic solutions of the two-body problem described above; see, for example, [25, 26]. We shall discuss these solutions in more detail in Remark 7.5.2. The union $N=N^{6}$ of the trajectories of these solutions is a 6-dimensional symplectic integrability submanifold in the 6(s-1)-dimensional phase space $M$. If $H<0$ and $\overrightarrow{\mathcal{M}} \neq 0$, where $H$ and $\overrightarrow{\mathcal{M}}$ are the total energy and vector of angular momentum, respectively, over all bodies of the system, then all the trajectories on $N=N^{6}$ are periodic. The solutions corresponding to these trajectories satisfy all the generalizations of Gordon's theorem, that is, the period and action depend only on the total energy $H$.

The simplest example is given by the well-known "triangular" Lagrange solutions in the case $s=3$, which correspond to the arrangement of the bodies at the vertices of an equilateral triangle moving along ellipses as in the case $s=2$; see, for example, [18. They generalize the circular Lagrange motions mentioned in $\S 1.1$, so that $N^{6} \supset N^{4}$, where $N^{4}$ corresponds to those circular motions. As in the case $s=2$, there is the fifth integral on $N^{6}$; the question of whether this integral can be continued to the entire phase space $M$ is of interest. The answer is most likely known, but the author does not know it.

\section{LiOUVILLE FOLIATIONS AND INTEGRABILITY OF SYSTEMS OF GENERAL FORM}

In this section we explain the general concept of Liouville integrability, which is suitable for systems of general form, rather than ones that are necessarily Hamiltonian. In $\S \S 2.1$ and 2.2 we define local, global and, strongest and most important, toric integrability; see Definitions 2.1.3 and 2.2.1. There we also briefly remind the reader how local (Proposition 2.1.1) and semilocal (Proposition 2.2.1) coordinates are constructed that normalize a vector field that is integrable locally or torically, respectively.

Finally, in $\S 2.3$ we consider the case when a vector field has, generally speaking, a nonabelian local Lie group of symmetries. We discuss one of the main and simplest methods of finding the local integrability submanifolds in the phase space of the field in this situation - specifying them by the sliding condition. Under certain natural assumptions, in the case where the group is compact and its action is global the integrability is toric.

2.1. Basic notions. All the functions, maps and manifolds throughout the paper are assumed to be smooth, that is, infinitely differentiable. By submanifolds $N \subseteq M$ we shall always mean, if not stipulated otherwise, the images of injective immersions of manifolds; 
these $N$ will also be called locally flat submanifolds. For example, as submanifolds we allow "irrational" windings of a torus, in particular, trajectories $N$ of the system $\dot{\phi}_{1}=\omega_{1}$, $\dot{\phi}_{2}=\omega_{2}$ lying on the 2-dimensional torus $M=\mathbf{T}^{2}$, where the frequency ratio $\omega_{2} / \omega_{1}$ is an irrational number. But we do not allow self-intersections, for example, eights.

Apart from locally flat submanifolds, we shall also consider "ordinary" submanifolds $N$, that is, where each point $m \in N$ has a neighbourhood $U$ in the whole of $M$ whose intersection $U \cap N$ with $N$ is defined in some coordinates in $U$ as a part of the coordinate subspace. Thus, here not only eights are excluded but also irrational windings of a torus. Such submanifolds are called embedded.

Suppose that on an arbitrary manifold $N$ we are given $k$ vector fields $a_{1}, \ldots, a_{k}$ that are linearly independent at each point $m \in N$ and are pairwise commutative on $N$ : $\left[a_{i}, a_{j}\right]=$ $0, i, j=1, \ldots, k$, where $[$,$] is the Lie bracket of vector fields. Linear combinations of the$ vectors of these fields define a $k$-dimensional distribution on $N$, that is, a field of planes, which we denote by $\theta=\theta(A)$, where $A=\left(a_{1}, \ldots, a_{k}\right)$. By Frobenius's theorem this distribution is integrable, that is, it defines a partition of the manifold $N$ into pairwise disjoint $k$-dimensional locally flat connected submanifolds $\Lambda$ which have the following property. At every point $m \in N$ the tangent plane $T_{m} \Lambda$ to the surface $\Lambda \ni m$ passing through this point coincides with the plane $\theta_{m}$ of the field $\theta$ at this point: $T_{m} \Lambda=\theta_{m}$.

The submanifolds $\Lambda$ are called maximal integral surfaces of the distribution $\theta$ and the partition $N=\bigcup \Lambda$ into these surfaces is called a foliation with leaves $\Lambda$. In other words, if a distribution is integrable, then it defines a foliation. The partition of the manifold $N$ into the (whole) trajectories of an arbitrary vector field $V$ that has no singular points on this manifold, that is, that does not vanish on $N$, is the one-dimensional foliation corresponding to a distribution of the type $\theta=\theta(A)$ described above. Here $V=a_{1}$ is the only field in the set $A$.

Definition 2.1.1. The foliation corresponding to the distribution $\theta=\theta(A)$ is called the Liouville foliation of the manifold $N$ defined by the set $A=\left(a_{1}, \ldots, a_{k}\right)$ of pairwise commuting vector fields on $N$. We denote this foliation by $\Xi=\Xi(A)$.

Let $F: N \rightarrow B$ be a map of the manifold $N$ into some manifold $B$. Recall that the rank of the map $F$ at a point $m_{0} \in N$, rank $F_{*}\left(m_{0}\right)$, is the rank of the matrix $\frac{\partial y}{\partial x}\left(x_{0}\right)$, where $y=y(x)$ is a vector-valued function defining the map $F$ in local coordinates $x$ in a neighbourhood of the point $m_{0}$ and in local coordinates $y$ defined in a neighbourhood of the point $b_{0}=F\left(m_{0}\right) \in B$, with the coordinates of the point $m_{0}$ given by $x_{0}$. This definition is independent of the choice of the local coordinates $x$ and $y$. If the rank of $F$ is constant at all the points $m \in N$, then it is called the rank of the map $F$, and we denote it by $\operatorname{rank} F_{*}$. A map $F$ is said to be regular at a point $m \in N$ (or on the whole of $N$ ) if it has maximum rank, that is, $\operatorname{rank} F_{*}(m)=\mu$ (or $\operatorname{rank} F_{*}=\mu$ ), where $\mu=\min \{\operatorname{dim} N, \operatorname{dim} B\}$.

All these definitions can also be extended to an arbitrary set of functions on $N$, $F=\left(F_{1}, \ldots, F_{r}\right)$, since such a set defines a map $F: N \rightarrow \mathbf{R}^{r}$.

Suppose that the partition of the manifold $N$ into the connected components of the inverse images $F^{-1}(b)$ of points $b \in B$ under some map $F: N \rightarrow B$ that is regular on the whole of $N$ coincides with the partition of $N$ into the leaves of a given Liouville foliation $\Xi(A)$.

Definition 2.1.2. In this case we call the Liouville foliation $\Xi(A)$ a Liouville fibration defined by the set of vector fields $A=\left(a_{1}, \ldots, a_{k}\right)$ and the regular map $F$ and denote it by $\Xi(A, F)$ or simply by $\Xi(A)$. In this situation the fibring map $F: N \rightarrow B$ is often called an invariant map or a momentum map. We define the fibres of the Liouville 
fibration not as the inverse images $F^{-1}(b) \subset N, b \in B$, themselves but as their connected components $\Lambda$. Note that in this situation we have $k+\operatorname{dim} B=\operatorname{dim} N$.

Suppose that $V$ is a vector field on a manifold $N$ and in addition there is a set $A=\left(a_{1}, \ldots, a_{k}\right)$ of vector fields on $N$ having the following properties.

a) The field $V$ commutes with all the fields in the set $A:\left[V, a_{i}\right]=0, i=1, \ldots, k$, or, for short, $[V, A]=0$.

b) At each point $m \in N$ the vector $V(m)$ of the field $V$ is a linear combination of vectors of the fields in the set $A$ :

$$
V(m)=\sum_{i=1}^{k} \lambda_{i}(m) a_{i}(m) .
$$

c) The set $A$ defines a Liouville foliation of the manifold $N$, that is, $[A, A]=0$ and the vectors of the fields in the set $A$ are linearly independent at each point of $N$.

Definition 2.1.3. In this case we say that the vector field $V$ has an abelian set of sliding symmetries $A$. If the set $A$ together with a map $F: N \rightarrow B$ defines a Liouville fibration, then we say that the field $V$ is $(A, F)$-integrable or simply A-integrable on $N$. Furthermore, in the case of foliations we may speak about the local integrability of the system, and in the case of a fibration, about its global integrability. We call condition b) the sliding condition.

Note that if one of the fields $a_{i}$ in the set $A$ defining a Liouville foliation of $N$ (condition c)) is taken to be $V$, then parts a) and b) of the definition are satisfied automatically. We also note that if the field $V$ has a set of abelian symmetries $A=\left(a_{1}, \ldots, a_{k}\right)$, then all the fields in the set $\left(V, a_{1}, \ldots, a_{k}\right)$ of $k+1$ fields extended by $V$ are pairwise commutative.

In addition we note that locally, that is, in some neighbourhood $U$ of any point $m_{0} \in N$, any foliation is a fibration; hence any field $V$ with some set of abelian symmetries $A$ is $A$-integrable in $U$. Indeed, locally the leaves of any foliation can be defined as the inverse images of a regular map of the form $F: U \rightarrow \mathbf{R}^{r}$, where $r$ is the codimension of the leaves, and therefore the field $V$ is $(A, F)$-integrable in $U$.

The term "integrability" is used for the following reason. One can show that the solutions of the system of ordinary differential equations corresponding to a vector field $V$ integrable in this sense can be expressed by quadratures in terms of the right-hand sides of the equations of this system and the systems corresponding to the fields $a_{1}, \ldots, a_{k}$ and in terms of the functions defining the fibring map $F$. Furthermore, the integrability of the field $V$ ensures relatively simple motion in this vector field. In particular, we have the following simple assertion.

Proposition 2.1.1 (Local normalizing coordinates). Let $\Xi(A, F)$ be an arbitrary Liouville fibration of a manifold $N$ defined by a set of vector fields $A=\left(a_{1}, \ldots, a_{k}\right)$ and a map $F: N \rightarrow B$. Then in some neighbourhood $U$ in $N$ of any point $m_{0} \in N$ there exist functions $\tau_{1}, \ldots, \tau_{k}$ with the following property. Together with the functions in the set $F=\left(F_{1}, \ldots, F_{r}\right)$ defining the map $F$ in some local coordinates $y=\left(y_{1}, \ldots, y_{r}\right)$ on $B$, where $r=\operatorname{dim} B$, they form coordinates in $U \subseteq N$ with the following properties.

A) In the coordinates $(F, \tau)$, where $\tau=\left(\tau_{1}, \ldots, \tau_{k}\right)$, for each $i=1, \ldots, k$ the vector field $a_{i}$ is defined by the system of $k+r$ differential equations $\dot{F}=0, \dot{\tau}_{j}=\delta_{i j}$, $j=1, \ldots, k$, where $\delta_{i j}$ is the Kronecker delta.

B) Suppose that $V$ is any vector field commuting with all the fields in the set $A$ : $[V, A]=0$. Then in the local coordinates $(F, \tau)$ it is defined by the system of ordinary differential equations $\dot{F}=C(F), \dot{\tau}=D(F)$, where $C(F)$ and $D(F)$ are some vector-valued functions independent of $\tau$. In particular, if $V$ is any 
$(A, F)$-integrable vector field, then it is defined by the system of equations $\dot{F}=0$, $\dot{\tau}=D(F)$.

Note that assertion A) can be interpreted as a theorem on the existence of local straightening coordinates for a set $A$ of pairwise commutative vector fields in a neighbourhood of a nonsingular point, that is, a point at which the vectors of these fields are linearly independent. The coordinates exist by A), using the fact that a set $F=\left(F_{1}, \ldots, F_{r}\right)$ of local functions with $r+k=\operatorname{dim} N$ invariant under the phase flows of all these fields exists, as we noted above.

The construction of the normalizing coordinates $(F, \tau)$ is carried out explicitly with respect to $F$ and $A$. Namely, the functions in the sets $\tau$ are expressed by quadratures in terms of the function in the set $F$ and the right-hand sides of the systems of equations defining the vector fields in the set $A$ in some local coordinates. If $V$ is an $(A, F)$-integrable field, then we can constructively find the form of this field in the coordinates $(F, \tau)$. More precisely, the vector-valued function $D(F)$ is expressed by quadratures in terms of the functions in the set $F$ and the right-hand sides of the systems of equations defining the vector fields in the set $A$ and the field $V$.

Proof of Proposition 2.1.1. The proof can be extracted, for example, from [1] or [5]. We remind the reader of this proof. We begin with $\mathrm{A}$ ). To construct the functions $\tau_{1}, \ldots, \tau_{k}$, given $m_{0} \in N$ we must construct an $r$-dimensional local transversal $\Gamma$ to the inverse image $F^{-1}\left(b_{0}\right)$ passing through this point, where $b_{0}=F\left(m_{0}\right)$. Then we set $\tau=0$ on $\Gamma$, and at the points $m \in N$ close to $\Gamma$ we define $\tau(m)$ to be equal to the "multi-dimensional time" $t=\left(t_{1}, \ldots, t_{k}\right)$ that has to be spent by the "multi-dimensional flow" $g^{t}$ to carry a point $m^{\prime} \in \Gamma$ to the given point $m$, that is, we define $\tau(m)$ so that $g^{\tau(m)} m^{\prime}=m$. Here $g^{t}=g_{k}^{t_{k}} \circ g_{k-1}^{t_{k-1}} \circ \cdots \circ g_{1}^{t_{1}}$ is the composite of the maps $g_{i}^{t_{i}}$, where $g_{i}^{t_{i}}$ is the transformation over time $t_{i}$ of the local phase flow defined on $N$ by the vector field $a_{i}, i=1, \ldots, k$. Assertion A) is easily obtained from the construction of the functions $\tau_{i}$.

To prove B) we express $V$ in the coordinates we have constructed, $(F, \tau): \dot{F}=C(F, \tau)$, $\dot{\tau}=D(F, \tau)$. For each $i=1, \ldots, k$ the field $V$ commutes with the vector field defined by the system of $k+r$ differential equations $\dot{F}=0, \dot{\tau}_{j}=\delta_{i j}, j=1, \ldots, k$. Using this and the expression for the Lie bracket in these coordinates we obtain that the vector-valued functions $C(F, \tau)$ and $D(F, \tau)$ are independent of $\tau$. The proof of Proposition 2.1.1 is complete.

2.2. Toric fibrations and toric integrability. In what follows we shall mainly be interested in Liouville fibrations, rather than just Liouville foliations. In particular, we concentrate on the case when all their fibres, that is, the connected components $\Lambda$ of all the inverse images $F^{-1}(b)$ of points $b \in B$, are compact.

Definition 2.2.1. Under the hypotheses above, the Liouville fibration $\Xi(A, F)$ of the manifold $N$ defined by a set of vector fields $A=\left(a_{1}, \ldots, a_{k}\right)$ on $N$ and a map $F: N \rightarrow B$ is called a toric fibration. If in addition there is a vector field $V$ on $N$ having a set of symmetries $A$, then we say that the field $V$ is torically $(A, F)$-integrable or torically A-integrable.

Using the term "torically" is justified by the following proposition.

Proposition 2.2.1. Let $\Xi=\Xi(A, F)$ be an arbitrary toric fibration of a manifold $N$, where $A=\left(a_{1}, \ldots, a_{k}\right), k \geq 1, \operatorname{dim} B=r \geq 0$, and correspondingly $\operatorname{dim} N=k+r$. Then the following holds.

A) Every connected component $\Lambda$ of the inverse image $F^{-1}(b)$ of any point $b \in B$ under the map $F: N \rightarrow B$ is a $k$-dimensional torus and the partition of $N$ into 
such tori defines a locally trivial fibration of $N$. Moreover, in some neighbourhood $U$ in $N$ of each component $\Lambda^{0} \subseteq N$ we can define $k$ "angular functions"

$$
\varphi_{1} \bmod 2 \pi, \ldots, \varphi_{k} \bmod 2 \pi
$$

with the following properties.

The set $(F, \varphi \bmod 2 \pi)$ with $\varphi=\left(\varphi_{1}, \ldots, \varphi_{k}\right)$ defines a set of "coordinates" in $U$, that is, this set defines a diffeomorphism $U \rightarrow D^{r} \times \mathbf{T}^{k}$ in the domain $U \subseteq N$ onto the direct product $D^{r} \times \mathbf{T}^{k}$. Here $D^{r}$ is a domain of the linear space $\mathbf{R}^{r}$; when $r=0$ it is a point; $\mathbf{T}^{k}$ is a $k$-dimensional torus. Furthermore, the restrictions of the "functions" in the set $\varphi \bmod 2 \pi$ to each torus $F^{-1}(b) \cap U$ are "angular coordinates" on this torus. In addition, each vector field $a_{i}, i=$ $1, \ldots, k$, in these "coordinates" is given by a system of equations of the form $\dot{F}=0, \dot{\varphi}=\Omega^{i}(F)$ defining a conditionally periodic motion on the invariant tori $\Lambda=F^{-1}(b) \cap U$.

B) Suppose that in addition we have an arbitrary torically $(A, F)$-integrable system. Then in these coordinates it is defined by a system of equations of the same type: $\dot{F}=0, \dot{\varphi}=\Omega(F)$.

Proof of Proposition 2.2.1. We sketch the proof. We take it that the construction of the normalizing "coordinates" $\varphi \bmod 2 \pi$ is a fairly well-developed element of the proof of the Liouville-Arnol'd theorem and its generalizations, so the missing details can be extracted, for example, from [1]; see also [5]. One needs to "forget" the Hamiltonian nature of the case considered there, which makes it much simpler. We give a short proof of this simple fact because it is essential later on in the paper.

The fact that each connected surface $\Lambda$ is diffeomorphic to the $k$-dimensional torus follows because it is compact and there exist $k$ pairwise commutative vector fields $a_{1}, \ldots, a_{k}$ defined on it and linearly independent at each point $m \in N$. The "coordinates" $\varphi \bmod 2 \pi$ on each fixed torus $\Lambda$ are constructed as in the proof of Proposition 2.1.1, that is, as the "multi-dimensional" time $\varphi$ defined by the abelian group $g^{\varphi}, \varphi \in \mathbf{R}^{k}$, which is the composite of the flows of pairwise commutative vector fields. Now, we do not take the $a_{1}, \ldots, a_{k}$ themselves for these fields but linear combinations of them, $a_{i}^{\prime}=\sum_{j=1}^{k} \lambda_{i j} a_{j}$, $i=1, \ldots, k$, with constant coefficients depending only on the torus $\Lambda$ under consideration.

These coefficients $\lambda_{i j}$ are chosen so that all the trajectories of each of these fields $a_{i}^{\prime}$ are periodic on $\Lambda$ with period $2 \pi$, and taken together over all $i=1, \ldots, k$ the trajectories form a basis of cycles in the fundamental group $\pi_{1}(\Lambda)$ of the torus $\Lambda$. This implies that the square matrix $\left(\lambda_{i j}, i, j=1, \ldots, k\right)$ is nonsingular and therefore the vector fields $a_{1}^{\prime}, \ldots, a_{k}^{\prime}$ are linearly independent at each point $m \in \Lambda$. Thus, the "angular coordinates" $\varphi \bmod 2 \pi$ on each torus $\Lambda$ are determined by the choice of a basis $\gamma_{1}, \ldots, \gamma_{k}$ in the group $\pi_{1}(\Lambda) \cong \mathbf{Z}^{k}$ and by the choice of the initial point $\varphi \bmod 2 \pi=0$.

Since the compact surfaces $\Lambda$ are connected components of the inverse images $F^{-1}(b)$ of points under the regular map $F$, these surfaces define a locally trivial fibration of the manifold $N$. In other words, each such torus $\Lambda^{0}$ has a neighbourhood $U$ in $N$ diffeomorphic to the direct product of the torus $\mathbf{T}^{k}$ and the disc $D^{r} \subset \mathbf{R}^{r}$ and this diffeomorphism $\psi: U \rightarrow D^{r} \times \mathbf{T}^{k}$ is compatible with the fibration of $U$ into the tori $\Lambda$, that is, $\psi^{-1}\left(\{b\} \times \mathbf{T}^{k}\right)=\Lambda^{b}=F^{-1}(b) \cap U$ for all $b \in F(U) \subset B$.

This trivial fibration allows one to choose, consistently over all the tori $\Lambda^{b} \subseteq U$, a set of basis cycles $\gamma_{1}, \ldots, \gamma_{k}, \gamma_{i}=\gamma_{i}(b) \in \pi_{1}\left(\Lambda^{b}\right)$, in the fundamental groups $\pi_{1}\left(\Lambda^{b}\right)$ of the tori $\Lambda^{b}$. First we must choose a basis in $\pi_{1}\left(\mathbf{T}^{k}\right)$ and then carry it over to the groups $\pi_{1}\left(\Lambda^{b}\right)$ by the map $\psi^{-1}$. It is clear that then the coefficients $\lambda_{i j}=\lambda_{i j}\left(\Lambda^{b}\right)$ will depend smoothly on the torus $\Lambda^{b}$, that is, on the point $b \in F(U)$. 
In order to choose the initial point $\varphi \bmod 2 \pi=0$ to be compatible over all the tori $\Lambda \subset U$ through any point $m_{0} \in \Lambda^{0}$ we must construct an $r$-dimensional transversal $\Gamma$ to the fibre $\Lambda^{0}$ and set $\varphi \bmod 2 \pi=0$ on $\Gamma(\operatorname{codim} \Lambda=r$ in $N)$, as in the proof of Proposition 2.1.1. As a result we obtain a set $\varphi \bmod 2 \pi$ of "functions" defined on the whole of $U$. It is clear that the "functions" $(F, \varphi \bmod 2 \pi)$ globally defined on $U$ are "coordinates" on $U$. Thus, the construction of the "coordinates" $(F, \varphi \bmod 2 \pi)$ in $U$ is complete. That $\varphi$ is independent of the right-hand sides of the equations defining the vector fields $V$ and $a_{1}, \ldots, a_{k}$ in these "coordinates" follows, as in the proof of Proposition 2.1.1, from the fact that these fields are pairwise commutative on the tori $\Lambda$. Proposition 2.2.1 is proved.

2.3. Finding the integrability submanifolds of systems with nonabelian symmetries. Let $V$ be an arbitrary vector field defined on some manifold $M$. In many cases the existence of abelian sliding symmetries for this field and the fact that it is integrable, which hold at least on some invariant submanifold $N \subseteq M$, are due to nonabelian symmetries of the field $V$ on the whole of $M$. We focus briefly on finding such submanifolds, especially those on which the system is torically integrable.

Let $\gamma$ be some finite-dimensional, in general nonabelian Lie subalgebra of the Lie algebra $\operatorname{Vect}(M)$ of all smooth vector fields on the manifold $M$ with the Lie bracket of vector fields as the commutator operation in this algebra.

Definition 2.3.1. We say that $\gamma$ is a symmetry algebra of the vector field $V$ defined on $M$ if $[V, a]=0$ for any field $a \in \gamma$. Then the field $V$ itself is said to be $\gamma$-symmetric. Suppose that in addition at each point $m \in M$ for some linear basis $a_{1}, \ldots, a_{s}$ of $\gamma$ we have the sliding condition:

$$
V(m)=\sum_{i=1}^{s} \lambda_{i}(m) a_{i}(m)
$$

cf. condition b) of Definition 2.1.3. Then we say that $\gamma$ is an algebra of sliding symmetries of the field $V$.

Note that there is complete compatibility between Definition 2.1.3 and this definition in the case where the algebra $\gamma$ is abelian and such that the local Lie group $G=G(\gamma)$ corresponding to this Lie algebra acts locally freely at all the points of $M$. Indeed, we can assume that in $\S 2.1$ we considered a linear basis $A=\left(a_{1}, \ldots, a_{k}\right)$ of such a $k$-dimensional abelian Lie algebra of vector fields on $N$.

Now consider the case where a symmetry algebra $\gamma$ is not an algebra of sliding symmetries on the whole of $M$. Then an integrability submanifold $N \subseteq M$ can often be specified in the whole phase space $M$ by the sliding condition; see (1). The assertions of this subsection explain to some extent the appearance of such integrability. The following Proposition 2.3.1 asserts the invariance of the submanifold specified in this way, and Proposition 2.3.2 shows, in particular, how an abelian symmetry group can emerge in a nonabelian situation.

Proposition 2.3.1 (Invariance of the submanifold specified by the sliding condition). Suppose that a vector field $V$ is defined on some manifold $M$ having a symmetry Lie algebra $\gamma$. Consider the subset $N \subseteq M$ specified in $M \ni m$ by the sliding condition (11), where $a_{1}, \ldots, a_{s}$ is a linear basis of $\gamma$. Then this subset is invariant under the action of the local group $G=G(\gamma)$. In other words, if $N$ intersects some orbit of this group, then it contains the whole orbit.

Suppose in addition that $N$ is a smooth submanifold of $M$. Then the vector field $V$ and all the fields $a \in \gamma$ are tangent to $N$ at all the points $m \in N$. Let $\tilde{V}$ and $\tilde{a}$ denote 
the restrictions of these fields to $N$; then the Lie algebra $\tilde{\gamma}$ isomorphic to $\gamma$ that consists of the fields $\tilde{a}:=\left.a\right|_{N}$ for all $a \in \gamma$ is an algebra of sliding symmetries of the field $\tilde{V}$. The submanifold $N$ specified by the sliding condition is maximal among all the invariant submanifolds on which the field $\left.V\right|_{N}$ has the algebra of sliding symmetries $\left.\gamma\right|_{N}:=\tilde{\gamma}$.

Furthermore, if the algebra $\gamma$ is abelian and the local Lie group $G=G(\gamma)$ acts locally freely at all the points of $N$, then the field $\tilde{V}$ is locally $\tilde{A}$-integrable on the whole of $N$, where $\tilde{A}$ is any linear basis of $\tilde{\gamma}$.

Proof of Proposition 2.3.1. Let $m \in M$ be an arbitrary point and consider the orbit $O=O_{m}$ of this point under the action of the group $G$. By the sliding condition the vector $V(m)$ is tangent to $O$. Since $[V, \gamma]=0, V$ is invariant under the action of $G$. Hence the restricted field $\left.V\right|_{O}$ is obtained by spreading the vector $V(m)$ by the group $G$ over the orbit $O$. Consequently, $\left.V\right|_{O}$ is tangent to the orbit $O$ at all points $m \in O$ and therefore $O_{m} \subseteq N$. Thus, $N$ is invariant under the action of the group $G$. The sliding condition implies that this is also true for the phase flow of the field $V$. The commutativity conditions for the vector fields are preserved on an invariant submanifold. This implies all the remaining assertions of Proposition 2.3.1 and completes the proof.

Thus, in the case of an abelian symmetry algebra $\gamma$ the position regarding integrability on the submanifold $N$ specified by the sliding condition (11) is fairly clear. We now present arguments needed to understand the nonabelian case.

For the moment we will assume that $\gamma$ is an arbitrary abstract Lie algebra, rather than necessarily an algebra of vector fields. Let $\breve{\gamma} \subseteq \gamma$ be some subset of the algebra $\gamma$ satisfying the following conditions. To each element $a \in \breve{\gamma}$ there corresponds an abelian subalgebra $\alpha(a) \subseteq \gamma$ containing the element $a$ and having the maximality property, that is, $\alpha(a)$ does not embed into any larger abelian subalgebra of the algebra $\gamma$. One may call such a subalgebra $\alpha(a)$ a maximal abelian extension of the element $a \in \gamma$. We also require that the family of subalgebras $\alpha(a)$ depends smoothly on the element $a \in \breve{\gamma}$ and is Ad-invariant. The latter means that the local Lie group $G=G(\gamma)$ corresponding to the Lie algebra $\gamma$ is invariant under the adjoint action on $\gamma$. More precisely, this means that for any $a \in \breve{\gamma}$ and $g \in G$ we must have $\operatorname{Ad}_{g} \alpha(a)=\alpha(b)$, where $b=\operatorname{Ad}_{g} a$. In particular, this means that the subset $\breve{\gamma} \subseteq \gamma$ itself is also Ad-invariant.

Definition 2.3.2. In this case we call $\breve{\gamma}$ an $\alpha$-regular subset of the algebra $\gamma$, and $\alpha$ itself a regular family of maximal abelian extensions of elements in $\breve{\gamma}$. Any element $a \in \breve{\gamma}$ of the Lie algebra $\gamma$ is said to be $\alpha$-regular. Suppose that the Lie algebra $\gamma$ is such that for some $\alpha$ there exists an $\alpha$-regular subset $\breve{\gamma}$ that is open and everywhere dense in $\gamma$. Then we say that a typical element of the algebra $\gamma$ is regular.

Remark 2.3.1. One can hope that a typical element of any Lie algebra $\gamma$ is regular. At least this is true for semisimple Lie algebras. For any semisimple algebra $\gamma$, the regular elements are the elements $a \in \gamma$ such that the dimension of the subalgebra $\operatorname{Ann}(a):=\{b \in \gamma \mid[b, a]=0\}$ of all elements $b \in \gamma$ commuting with $a$ is minimized. For such $a$ this subalgebra is always abelian, obviously contains $a$, is maximal, and Ad-invariant. (For such $a$ this $\operatorname{Ann}(a)$ is called a Cartan subalgebra. Its dimension is called the rank of the Lie algebra $\gamma: \operatorname{rank} \gamma:=k$, where $k=\min _{a \in \gamma}\{\operatorname{dim} \operatorname{Ann}(a)\}$.) The subset of all irregular elements is specified in $\gamma$ by algebraic conditions and therefore is closed and nowhere dense in $\gamma$. Furthermore, on the subset of regular elements of the Lie algebra $\gamma$ the subalgebra $\operatorname{Ann}(a)$ depends smoothly (and even analytically) on the parameter $a$. Hence one can take $\operatorname{Ann}(a)$ for $\alpha(a)$ in Definition 2.3.2.

It would be interesting to know what the structure of maximal abelian Lie subalgebras $\alpha(a) \ni a$ of an arbitrary Lie algebra $\gamma$ is like and whether it is true that a typical element of such an algebra is regular in the sense of Definition 2.3.2. 
Let $V$ be an arbitrary vector field defined on some manifold $N$, and now let $\gamma$ be some Lie algebra of sliding symmetries of this field, which in general will be nonabelian. We assume that the local Lie group $G=G(\gamma)$ corresponding to this Lie algebra acts locally freely at all the points of $N$. For each point $m \in N$, let $a_{m}$ denote an element of the algebra $\gamma$ such that $a_{m}(m)=V(m)$. By the sliding condition such an element $a_{m} \in \gamma$ does exist and under the condition that the action of the group $G=G(\gamma)$ is locally free, it is unique. Let $\alpha$ be some regular family of abelian extensions of the elements of the subset $\breve{\gamma} \subseteq \gamma$.

Definition 2.3.3. We say that a point $m \in N$ is an $\alpha$-regular point of the vector field $V$ if $a_{m}$ is an $\alpha$-regular element of the Lie algebra $\gamma$, that is, $a_{m} \in \breve{\gamma}$.

Proposition 2.3.2 (Integrability of $\alpha$-regular vector fields). A) Suppose that a vector field $V$ is defined on some manifold $N$ having an algebra of sliding symmetries $\gamma$. Suppose that $\gamma$ has a regular family of maximal abelian extensions $\alpha=\{\alpha(a), a \in \dot{\gamma}\}$ such that each point $m \in N$ is an $\alpha$-regular point of the field $V$. For each point $m \in N$, let $\mathcal{G}_{m}$ denote the abelian Lie subgroup of the group $G=G(\gamma)$ corresponding to the subalgebra $\alpha\left(a_{m}\right) \subseteq \gamma$, and let $\Lambda_{m}$ denote the orbit of this subgroup passing through the point $m$ : $\Lambda_{m} \ni m$. Then the surfaces $\Lambda_{m}, m \in N$, define a smooth foliation of the manifold $N$. In particular, for any two points $p$ and $q$ in $N$ the surfaces $\Lambda_{p}$ and $\Lambda_{q}$ either are disjoint or coincide. Furthermore, the foliation into the submanifolds $\Lambda_{m}$ is locally invariant under the action of the entire group $G=G(\gamma)$.

B) Suppose in addition that the surfaces $\Lambda_{m}$ are compact and form a locally trivial fibration of the submanifold $N$. Then these surfaces are tori and there exists a neighbourhood $U$ in $N$ for each such torus in which the system is torically integrable. Moreover, the neighbourhood $U$ can be chosen so that there exist normalizing coordinates $(F, \varphi \bmod 2 \pi)$ in it with the following property. All the tori $\Lambda_{m} \subseteq U$ contained in $U$ have the form $\{F=$ const $\}$, and the field $V$ is given in $U$ in the form $\dot{F}=0, \dot{\varphi}=\Omega(F)$. Furthermore, the action of the subgroup $\mathcal{G}_{m}$ on its orbit $\Lambda_{m}$ for any $m \in U$ has the form of translations with respect to $\varphi$.

Remark 2.3.2. The condition that the fibres $\Lambda_{m}$ be compact and form a locally trivial fibration of $N$ may be considered to be the most restrictive one. However, this condition will be satisfied in the typical situation if the group $G=G(\gamma)$ is assumed to be compact and acting globally. Indeed, the condition that the abelian subalgebras $\alpha\left(a_{m}\right) \subseteq \gamma$ be maximal implies that the corresponding Lie subgroups $\mathcal{G}_{m}=\mathcal{G}\left(\alpha\left(a_{m}\right)\right), \mathcal{G}_{m} \subseteq G$, are closed. When $G$ is compact this implies that the Lie subgroups $\mathcal{G}_{m}$ for $m \in N$ are also compact. Since the action is global, the fibres $\Lambda_{m}$ of the fibration under consideration are also compact as orbits of these subgroups. Since the fibres $\Lambda_{m}$ are compact, this fibration must be trivial in a neighbourhood of a typical fibre.

This explains the significance of nonabelian compact Lie groups of symmetries, even not necessarily sliding ones, for integrability. We can assume that, as a rule, a vector field $V$ defined on an arbitrary submanifold of $M$ is tangent to one of the orbits $O$ of such a symmetry group $G$ at least at one point, that is, at this point the sliding condition holds. Then we have the nonempty submanifold $N \subseteq M$ specified by the sliding condition. If the restriction of $V$ to this $N$ is $\alpha$-regular on $N$, then $\left.V\right|_{N}$ is torically integrable in the typical situation.

In particular, suppose that some vector field $V$ has a compact symmetry group $G$ on $M$ such that a typical element of the corresponding Lie algebra $\gamma=\gamma(G)$ is regular in the sense of Definition 2.3.2. Then we can assume that, with the exception of the cases of total nontangency of the field $V$ to the orbits $O$ of this group (which we assume are rare), the following "principle" holds for such systems. In the typical case this field has at 
least one submanifold of toric integrability invariant under the action of $G$. Furthermore, the tori invariant under the flow of the field with conditionally periodic motion on them fibring this submanifold $N \subseteq M$ are orbits of the abelian subgroups of the group $G$. In addition, if the conjecture on the regularity of a typical element of the Lie algebra of any compact Lie group is true, cf. Remark 2.3.1, then this "principle" will be true for any compact symmetry group.

Note that it is not necessary that $G$ is the Lie group of all symmetries of a given field, which may well not be compact. What is important is that this full group must have at least one compact subgroup. Of course, in the passage from the group to a subgroup the submanifold $N$ specified by the sliding condition (11) can get correspondingly smaller or even disappear; see Proposition 2.3.1.

Proof of Proposition 2.3.2. The proof of assertion A) is based on the Ad-invariance of the abelian subalgebras $\alpha(a) \subseteq \gamma$ and the $G$-invariance of the vector field $V$. Consider the distribution $\theta$ on $N$ that is defined by the subalgebras $\alpha\left(a_{m}\right)$ for $m \in N$ as follows (recall that $\left.a_{m}(m)=V(m)\right)$. For $\theta_{m} \subseteq T_{m} N$ we take the plane $\theta_{m}=\left\{w \in T_{m} N \mid w=a(m)\right.$, where $\left.a \in \alpha\left(a_{m}\right)\right\}$, that is, the plane $\theta_{m}$ is formed by the vectors at the point $m$ of all the fields in the subalgebra $\alpha\left(a_{m}\right) \subseteq \gamma$. We claim that the field of planes $\theta$ is invariant under the action of the group $G=G(\gamma): g_{*} \theta_{m}=\theta_{g m}$ for any $m \in N$ and $g \in G$, where $g_{*}$ is the derivative of the map $g$.

To show this, we use the well-known formula $g_{*} a=\operatorname{Ad}_{g} a$, which is valid for any vector field $a \in \gamma$ and any element $g \in G(\gamma)$, where $\gamma$ is an arbitrary Lie algebra of vector fields. From this formula and the Ad-invariance of the subalgebras $\alpha(a)$ we obtain

$$
g_{*} \alpha\left(a_{m}\right)=\operatorname{Ad}_{g} \alpha\left(a_{m}\right)=\alpha\left(\operatorname{Ad}_{g} a_{m}\right)
$$

Due to the $G$-invariance of the original field $V$ we have $g_{*}(V)=V$; hence,

$$
\left(\operatorname{Ad}_{g} a_{m}\right)(g m)=\left(g_{*} a_{m}\right)(g m)=g_{*}\left(a_{m}(m)\right)=g_{*}(V(m))=V(g m)=\left(a_{g m}\right)(g m) .
$$

Since the group $G$ acts locally freely, if two fields in the algebra $\gamma$ coincide at one point in $N$, then they coincide as elements of the algebra; hence $\operatorname{Ad}_{g} a_{m}=a_{g m}$. These two equalities obtained imply

$$
g_{*} \theta_{m}=\left(g_{*} \alpha\left(a_{m}\right)\right)(g m)=\left(\alpha\left(\operatorname{Ad}_{g} a_{m}\right)\right)(g m)=\left(\alpha\left(a_{g m}\right)\right)(g m)=\theta_{g m} .
$$

Consequently, the distribution $\theta$ is indeed $G$-invariant.

We now show that the distribution $\theta$ is integrable, and for any $m \in N$ its integral surface $L_{m} \ni m$ coincides with the orbit $\Lambda_{m} \ni m$ of the subgroup $\mathcal{G}_{m}=\mathcal{G}\left(\alpha\left(a_{m}\right)\right)$ corresponding to the subalgebra $\alpha\left(a_{m}\right)$. To do this it is sufficient to show that for any $m \in N$ at any point $p \in \Lambda_{m}$ the tangent space $T_{p} \Lambda_{m}$ to the orbit $\Lambda_{m}$ coincides with $\theta_{p}$, that is, $T_{p} \Lambda_{m}=\theta_{p}$ for any $m \in N$ and $p \in \Lambda_{m}$. Consider an element $g \in \mathcal{G}_{m}=\mathcal{G}\left(\alpha\left(a_{m}\right)\right)$ such that $g m=p$. Since the subalgebra $\alpha\left(a_{m}\right)$ is abelian, we have $\operatorname{Ad}_{g} \alpha\left(a_{m}\right)=\alpha\left(a_{m}\right)$. Using a part of the calculation (2), the Ad-invariance of $\alpha$, and this equality we obtain

$$
\theta_{p}=\left(\alpha\left(\operatorname{Ad}_{g} a_{m}\right)\right)(p)=\left(\operatorname{Ad}_{g} \alpha\left(a_{m}\right)\right)(p)=\left(\alpha\left(a_{m}\right)\right)(p)=T_{p} \Lambda_{m}
$$

The smoothness of the foliation of $N$ into the leaves $\Lambda_{m}$ follows from the smoothness of the distribution $\theta$, which follows from the obvious fact that the subalgebras $\alpha\left(a_{m}\right)$ depend smoothly on the point $m \in N$. The local $G$-invariance of these leaves follows from the $G$-invariance of the distribution $\theta$. The proof of Proposition 2.3.2 A) is complete.

Proof of Proposition 2.3.2 B). The case $\operatorname{dim} \Lambda=\operatorname{dim} N$ is simple and is easily reduced to the case $\operatorname{dim} \Lambda<\operatorname{dim} N$, which we shall consider. Each fibre $\Lambda$ is a compact orbit of an abelian group and therefore is diffeomorphic to the torus. We fix any point $m \in N$. In some neighbourhood $U$ of the orbit $\Lambda_{m} \ni m$ of the subgroup $\mathcal{G}_{m}$ we construct a set $A=\left(a_{1}, \ldots, a_{k}\right)$ of $k$ vector fields which are pairwise commutative on $U$ and have the 
following properties. They are tangent to all the tori $\Lambda_{p} \subseteq U$ at all the points $p \in U$. Furthermore, the set $\bar{A}$ of the restrictions $\bar{a}_{i}:=\left.a_{i}\right|_{\Lambda_{p}}$ of these fields to each such torus is a linear basis of the abelian algebra $\overline{\alpha\left(a_{p}\right)}$ of the restrictions to $\Lambda_{p}$ of the fields of the subalgebra $\alpha\left(a_{p}\right)$. In particular, $k=\operatorname{dim} \Lambda_{p}$ and $\operatorname{span}(\bar{A})=\overline{\alpha\left(a_{p}\right)}$, where $\operatorname{span}(\bar{A})$ is the linear span of the fields in the set $\bar{A}$.

To construct the set $A$ we use the equality $\operatorname{Ad}_{g} a_{p}=a_{g p}$ proved above and the fact that the subalgebras $\alpha\left(a_{p}\right)$ are abelian, whence we obtain $a_{g p}=\operatorname{Ad}_{g} a_{p}=a_{p}$ for any $p \in N$ and $g \in \mathcal{G}_{p}$. Consequently,

$$
\alpha\left(a_{q}\right)=\alpha\left(a_{p}\right) \quad \forall q \in \Lambda_{p} .
$$

We choose a neighbourhood $U$ of the torus $\Lambda_{m}$ to be sufficiently narrow. Then the smoothness conditions and the equality obtained above imply that the algebras $\alpha\left(a_{p}\right)$ for all $p \in U$ are contained in a small neighbourhood of the subalgebra $\alpha\left(a_{m}\right) \subseteq \gamma$ in the corresponding Grassmannian manifold of $k$-dimensional subspaces of the linear space $\gamma$. Using this fact, the smoothness conditions and (3) one can easily obtain the existence of a basis $A_{q}$ in the subspace $\alpha\left(a_{q}\right) \subseteq \gamma$ that depends smoothly on the point $q \in U$ and is constant on each fibre $\Lambda_{p} \subset U$.

Consider the set $\bar{A}_{q}:=\left.A_{q}\right|_{\Lambda_{p}}$ of $k$ vector fields on $\Lambda_{p} \subset U$. Like $A_{q}$, the set $\bar{A}_{q}$ also does not depend on the choice of the point $q$ on the fibre $\Lambda_{p} \subset U$. Clearly it is a linear basis of the abelian algebra $\overline{\alpha\left(a_{q}\right)}, q \in \Lambda_{p}$. Taking the union over all $p \in U$ of the fields in this family of sets we obtain a set $A$ of vector fields defined on the entire domain $U$. It is clear that these fields are tangent to all the tori $\Lambda_{p} \subseteq U$. Since the basis $A_{p}$ depends smoothly on the point $p \in U$, the fields of the set $A$ are smooth. It is also clear that they are pairwise commutative on $U$ and therefore have all the properties that we required for the fields in the set $A$ above.

By the construction of the distribution $\theta$ the vector field $V$ "is contained" in $\theta$ and therefore it is tangent to all of its fibres $\Lambda_{p}$. On the other hand, for any $p \in N$ all the fields of the algebra $\alpha\left(a_{p}\right)$ commute with the field $V$, that is, $\left[V, \alpha\left(a_{p}\right)\right]=0$, since $\alpha\left(a_{p}\right)$ is a subalgebra of the symmetry algebra $\gamma$ of this field. Consequently, they will also commute on the invariant submanifold $\Lambda_{p}$. From this and the construction of $A$ we obtain that $[V, A]=0$ on $U$ and therefore the field $V$ is locally $A$-integrable on $U$. Using Proposition 2.2.1B) we obtain the existence of normalizing coordinates $(F, \varphi \bmod 2 \pi)$ with the required properties, which completes the proof of Proposition 2.3.2 B). The proof of Proposition 2.3.2 is complete.

Remark 2.3.3. In Proposition 2.3.2 B) we can omit the condition that the leaves $\Lambda_{m}$ be compact and that they form a locally trivial fibration of the manifold $N$, but then we can only obtain local $A$-integrability of the field $V$ on $N$ and just in some neighbourhood in $N$ of any point $m \in N$. Now $A=\left(a_{1}, \ldots, a_{k}\right)$ is some set of pairwise commuting vector fields defined only in this neighbourhood $\left(k=\operatorname{dim} \alpha\left(a_{m}\right)\right)$. To the set $A$ there corresponds a local abelian group $\mathcal{G}=\mathcal{G}_{A} \cong \mathbf{R}^{k}$. It follows from this construction of $A$ that neither the set $A$ nor the action of the group $\mathcal{G}$ are uniquely defined, but this arbitrariness is not essential.

The construction $(G, V) \longrightarrow \mathcal{G}$ of the group $\mathcal{G}$ from $G$ and $V$ may be called the "abelianization" of an arbitrary local group $G$ of sliding symmetries of a vector field $V$. When we have compactness, as in Proposition 2.3.2 B) we can take a compact group for $\mathcal{G}$, that is, a group isomorphic to the torus $\mathbf{T}^{k}$, which will act globally in an entire neighbourhood of the fibre $\Lambda$ that is an orbit of it.

The mechanism of integrability described above, which is related to the existence of an (in general) nonabelian symmetry group, is quite common. It lies behind the integrability 
of many concrete vector fields, both in general form and Hamiltonian, integrated over the entire phase space or on submanifolds of it; see $\S 1$ and $\S 7$.

\section{Some types of Hamiltonian integrable Systems AND SYSTEMS WITH SYMMETRIES}

In what follows we will just study the Hamiltonian case. In this section we will investigate three types of foliations and fibrations of submanifolds $N$ of symplectic manifolds $M$ : pseudo-Hamiltonian, weakly Hamiltonian, and simply Hamiltonian ones. Here the first ones mentioned are not even Liouville in the sense of $\S 2.1$, while the strongest conditions are imposed on the last, namely Hamiltonian; see Definitions 3.2.2 and 3.2.3. As in $\S 2$, these foliations will be defined by sets of vector fields, but now these are Hamiltonian. We also study the corresponding Hamiltonian systems, with phase space $M$ and a submanifold $N \subseteq M$ on which a foliation or fibration is defined with leaves (fibres) invariant under the phase flow of the system; see Definition 3.2.4. In the case of foliations they are called systems with symmetries on $N$, and in the case of fibrations, systems integrable on $N$.

Systems Hamiltonian-integrable on $N$, that is, systems with a corresponding Hamiltonian fibration, are to a great extent analogous to Hamiltonian systems integrable on a symplectic submanifold of the phase space $M$. In other words, this integrability on $N$ is the closest to the standard integrability of Hamiltonian systems.

In $\S \S 3.2-3.5$ we give the definitions of these foliations, fibrations, and systems of various types and study their properties and relations between them. In particular, Proposition 3.3.4 shows that in the case of a symplectic submanifold $N$, all three definitions: of a pseudo-Hamiltonian, weakly Hamiltonian, and Hamiltonian foliation, coincide.

The most important definitions are Definitions 3.2.2, 3.2.3, and 3.2.4. They are formulated uniformly so as to emphasize their connections with the corresponding definitions in $\S 2$ for systems of general form, that is, systems that are not necessarily Hamiltonian; therefore these definitions are not always "optimal". It is not easy to verify them for concrete systems, but even so it is convenient to use them. Some of the assertions in $\S 3.3-3.5$ show that particular conditions in these definitions are in fact redundant, and some can be reformulated more simply.

The central result in this section, Proposition 3.4.1 A) asserts that various definitions of Hamiltonian foliations are equivalent. In addition, in Corollary 3.4.3 and in Proposition 3.4.2 we touch on the question of finding the integrability submanifolds; see more details about this in $\S 7$.

3.1. Hamiltonian systems on symplectic manifolds. We recall some simple facts in the theory of Hamiltonian systems defined on symplectic manifolds; see, for example, 1 . By such a system we mean a triple $\left(M, \omega^{2}, H\right)$, where $M=M^{2 n}$ is a $2 n$-dimensional manifold equipped with a symplectic structure, that is, a nondegenerate closed differential 2 -form $\omega^{2}$, and $H: M \rightarrow \mathbf{R}$ is a function on $M$ called the Hamiltonian of the system. We denote the vector field on $M$ defining this system by $J d H$, although it is more often denoted by $X_{H}$; but the former notation is more convenient for us.

At each point $m \in M$, the vector $J_{m} d H$ of this field is defined by the relation $d H(\xi)=$ $\omega^{2}\left(\xi, J_{m} d H\right)$, which must hold for any vector $\xi \in T_{m} M$, where $T_{m} M$ is the tangent space to $M$ at this point. The linear map $J_{m}: T_{m}^{*} M \rightarrow T_{m} M$ acting on the conjugate space $T_{m}^{*} M$ of $T_{m} M$ is called the Hamiltonian operator at the point $m$, and the linear map $J$ from the space of all differential 1-forms on $M$ onto the space of all vector fields on $M$ is called the Hamiltonian operator on the symplectic manifold $\left(M, \omega^{2}\right)$. Note that both operators $J_{m}$ and $J$ define isomorphisms. 
On the space of all functions on $M$ the Poisson bracket of two functions $A$ and $B$ is defined:

$$
\{A, B\}=d A(J d B)=-d B(J d A)=\omega^{2}(J d B, J d A) .
$$

We shall also need the formula

$$
[J d A, J d B]=J d\{A, B\}
$$

which connects the Lie bracket $[J d A, J d B]$ of Hamiltonian vector fields $J d A$ and $J d B$ with the Poisson bracket $\{A, B\}$ of the functions $A$ and $B$ defining these fields.

Definition 3.1.1. A submanifold $N \subset M^{2 n}$ of a symplectic $2 n$-dimensional manifold $\left(M^{2 n}, \omega^{2}\right)$ is said to be isotropic if the restriction $\left.\omega^{2}\right|_{N}$ of the 2 -form $\omega^{2}$ to $N$ is equal to zero, $\tilde{\omega}^{2}=0$, where $\tilde{\omega}^{2}:=\left.\omega^{2}\right|_{N}$, and Lagrangian if it is isotropic and has the maximum possible dimension for isotropic submanifolds, that is, $\operatorname{dim} N=n$. A submanifold $N \subseteq M$ is said to be symplectic if the restricted 2 -form $\tilde{\omega}^{2}$ is nondegenerate. In other words, it is required that the pair $\left(N, \tilde{\omega}^{2}\right)$ be a symplectic manifold.

Thus, the dimension of an isotropic submanifold is at most $n$, and of a symplectic one is always even. We shall frequently use the following trivial fact.

Lemma 3.1.1 (Restriction to an invariant symplectic submanifold). Suppose that a system with Hamiltonian function $H$ is defined on a symplectic manifold $\left(M, \omega^{2}\right)$ and suppose that $N \subseteq M$ is a symplectic submanifold invariant under the local flow corresponding to this system, that is, $J_{m} d H \in T_{m} N$ at each point $m \in N$. Let $\tilde{J}$ denote the Hamiltonian operator corresponding to the symplectic manifold $\left(N, \tilde{\omega}^{2}\right)$ and let $\tilde{H}$ denote the restriction of the function $H$ to $N$. Then the following hold.

A) The restriction to $N$ of the Hamiltonian vector field JdH coincides with the Hamiltonian vector field $\tilde{J} d \tilde{H}$ :

$$
\left.(J d H)\right|_{N}=\tilde{J} d \tilde{H} .
$$

B) Suppose that $A$ is another function on $M$. Then

$$
\left.\{A, H\}\right|_{N}=\{\tilde{A}, \tilde{H}\}_{N},
$$

where $\tilde{A}=\left.A\right|_{N}$ and the Poisson bracket on the right $\{,\}_{N}$ is interpreted as corresponding to the symplectic manifold $\left(N, \tilde{\omega}^{2}\right)$. In particular, if the function $A$ is an integral of the original system on $M$, then the restriction $\tilde{A}=\left.A\right|_{N}$ is an integral of the system restricted to $N$.

We give the proof of this lemma for the sake of completeness.

Proof. We fix a point $m \in N$; then for any vector $\xi \in T_{m} N$ we have

$$
\tilde{\omega}^{2}(\xi, J d H)=\omega^{2}(\xi, J d H)=d H(\xi)=d \tilde{H}(\xi)=\tilde{\omega}^{2}(\xi, \tilde{J} d \tilde{H}) .
$$

This, with the fact that the 2-form $\tilde{\omega}^{2}$ is nondegenerate, implies that $J d H=\tilde{J} d \tilde{H}$ on $N$, which proves assertion A).

To prove B) we observe that on $N$ we have

$$
\{A, H\}=d A(J d H)=d A(\tilde{J} d \tilde{H})=d \tilde{A}(\tilde{J} d \tilde{H})=\{\tilde{A}, \tilde{H}\}_{N} .
$$

The proof of Lemma 3.1.1 is complete. 


\subsection{Definitions of Hamiltonian, weakly Hamiltonian, and pseudo-Hamiltonian} foliations and fibrations and of the corresponding systems.

Definition 3.2.1. An arbitrary foliation $\Xi$ of a submanifold $N \subseteq M$ of a symplectic manifold $\left(M, \omega^{2}\right)$ is said to be isotropic if all its leaves $\Lambda$ are isotropic in $M$, that is, $\left.\omega^{2}\right|_{\Lambda}=0$.

By a fibration of an arbitrary manifold $M$ we mean henceforth a partition of $M$ into the connected components $\Lambda$ of the inverse images $F^{-1}(b)$ of points $b \in B$ under some smooth map $F: M \rightarrow B$; cf. Definition 2.1.2. Here we shall always assume that a fibration is regular, that is, $F$ has no critical points. We call $F$ a fibring map; it is often called also a momentum map or invariant map.

Remark 3.2.0. In the case where all the fibres $\Lambda$ of such a fibration are compact, this is an ordinary locally trivial fibration. This simple fact was used in the proof of the Liouville-Arnol'd theorem, so its proof can be extracted from the proof of that theorem; see [1] and [5. It is proved there under the assumption that the fibres $\Lambda$ are tori, but this is not essential to the proof.

In the Hamiltonian case it is natural to consider Hamiltonian vector fields, rather than those of general form. Since such fields are defined by functions, in the Hamiltonian situation many things are more conveniently stated in terms of functions, rather than in terms of vector fields as in $\S 2$.

Let $Z=\left(Z_{1}, \ldots, Z_{k}\right)$ be a set of $k$ functions defined on a symplectic manifold $\left(M, \omega^{2}\right)$, and $N \subseteq M$ an arbitrary, not necessarily symplectic submanifold, and suppose that $Z$ and $N$ satisfy the following conditions.

a) (Z-invariance of $N$ ) The vector fields $J d Z_{1}, \ldots, J d Z_{k}$ defined by the functions in $Z$ are tangent to the submanifold $N$, that is,

$$
J_{m} d Z_{i} \in T_{m} N, \quad i=1, \ldots, k,
$$

at each point $m \in N$, where $J_{m} d Z_{i}$ is the vector of the field $J d Z_{i}$ at the point $m$.

Let $\tilde{Z}=\left(\tilde{Z}_{1}, \ldots, \tilde{Z}_{k}\right)$ denote the set of restrictions $\tilde{Z}_{i}:=\left.Z_{i}\right|_{N}$ to $N$ of the functions in the set $Z$. We require the following.

b) (Internal regularity of $Z$ ) The set $\tilde{Z}$ is regular on $N$ (see Definition 2.1.1), that is,

$$
\operatorname{rank} \tilde{Z}_{*}=k \text {. }
$$

In this case in some neighbourhood of the submanifold $N$ in $M$ the set $Z$ is obviously also regular. Consequently, at each point of this neighbourhood the vector fields $J d Z_{1}, \ldots, J d Z_{k}$ are linearly independent. We require the following.

c) (Commutativity of the fields $J d Z_{i}$ ) The vector fields $J d Z_{1}, \ldots, J d Z_{k}$ are pairwise commutative on $N$ :

$$
\left.\left[J d Z_{i}, J d Z_{j}\right]\right|_{N}=0, \quad i, j=1, \ldots, k .
$$

Let $\mathcal{J}_{N} d Z$ denote the field (defined on $N$ ) of the linear spans $\theta_{m}=\mathcal{J}_{m} d Z$ of the vectors of the fields $J d Z_{1}, \ldots, J d Z_{k}$ at points $m \in N$. It follows from (8) that the vector fields in the set $J_{N} d Z=\left(J_{N} d Z_{1}, \ldots, J_{N} d Z_{k}\right)$ consisting of the restrictions $J_{N} d Z_{i}:=\left.\left(J d Z_{i}\right)\right|_{N}$ to $N$ of the vector fields $J d Z_{i}$ commute pairwise. Since the vectors of these fields are pairwise commutative and linearly independent at each point $m \in N$, the distribution $\mathcal{J}_{N} d Z$ is regularly integrable. We denote by $\Xi_{N}(Z):=\Xi\left(J_{N} d Z\right)$ the $k$-dimensional Liouville foliation of the submanifold $N$ defined by this distribution; see Definition 2.1.1. We require the following.

d) (Isotropy of the foliation) The foliation $\Xi_{N}(Z)$ is isotropic, that is,

$$
\left.\omega^{2}\right|_{\Lambda}=0
$$

on each leaf $\Lambda$ of this foliation. 
Definition 3.2.2. If conditions a)-d) hold, then the Liouville foliation (fibration, toric fibration) $\Xi_{N}(Z)$ of the submanifold $N \subseteq M$ is called a Hamiltonian foliation (fibration, toric fibration) defined by the set $Z$ of functions on $M$.

We consider two more conditions, which are weaker than b) and c), respectively.

$\mathrm{b}^{\prime}$ ) (External regularity of $Z$ ) The set $Z=\left(Z_{1}, \ldots, Z_{k}\right)$ of functions on $M$ is regular on $N$, that is, $\operatorname{rank} Z_{*}(m)=k$ at all points $m \in N$.

$\left.c^{\prime}\right)$ (Frobenius integrability of the fields $J d Z$.) The field $\theta=\mathcal{J}_{N}(Z)$ of the linear spans of the vectors $J_{m} d Z_{1}, \ldots, J_{m} d Z_{k}$ tangent to $N$ is Frobenius integrable on $N$ and thus defines a $k$-dimensional foliation on $N$; it is not required that the fields $J d Z_{1}, \ldots, J d Z_{k}$ are pairwise commutative. By Frobenius' theorem this is equivalent to the following condition:

$$
\left[J d Z_{i}, J d Z_{j}\right](m)=\sum_{s=1}^{k} \lambda_{s}^{i j} J_{m} d Z_{s}
$$

at each point $m \in N$, where $\lambda_{s}^{i j}=\lambda_{s}^{i j}(m)$.

Definition 3.2.3. In the set of conditions a)-d), we replace condition b) concerning internal regularity by the weaker condition $b^{\prime}$ ) concerning external regularity. In this case the vector fields $J d Z_{1}, \ldots, J d Z_{k}$ as before will define a Liouville foliation, which may be a fibration or a toric fibration (see the definitions in $\S 2$ ); in all these cases we call this foliation weakly Hamiltonian.

If we also replace the commutation condition c) by Frobenius integrability $\mathrm{c}^{\prime}$ ), then the linear spans $\theta_{m}=\mathcal{J}_{m} d Z$ of the vectors of these fields will also define a foliation, which may not be Liouville, but may be a fibration or a toric fibration (that is, a fibration into tori). Then such foliations and fibrations are called pseudo-Hamiltonian and are also denoted by $\Xi_{N}(Z)$.

Remark 3.2.1. In the case of a fibration, that is, when there exist $\operatorname{dim} N-k$ functionally independent integrals on $N$ common to all $k$ fields in the set $J_{N} d Z$, condition $\mathrm{c}^{\prime}$ ) concerning the Frobenius integrability of the distribution $\theta=\theta\left(J_{N} d Z\right)=\mathcal{J}_{N} d Z$ is automatically satisfied.

Remark 3.2.2. An equivalent definition of a weakly Hamiltonian foliation is to define it as a $k$-dimensional isotropic Liouville foliation of $N$, in the sense of Definition 2.1.1, defined by the restrictions to $N$ of some $k$ Hamiltonian vector fields $J d F_{1}, \ldots, J d F_{k}$, that is, $\Xi=\Xi\left(J_{N} d F\right)$. The requirement that the foliation be isotropic can be replaced by the equivalent condition $\left.\{F, F\}\right|_{N}=0$ of pairwise involutivity of the functions in the set $F=\left(F_{1}, \ldots, F_{k}\right)$ on $N$; see more details in Proposition 3.3.3.

Remark 3.2.3. If a foliation is weakly Hamiltonian, this does not imply it is Hamiltonian - this is shown by the example of a fibration given in $\S 5.4$; the same example is a counterexample to Gordon's theorem. We also note that the regularity of $\tilde{Z}$ (condition b)) follows from the regularity of $Z$ on $N$ (condition $\left.b^{\prime}\right)$ ) if and only if at each point $m \in N$ the tangent planes $T_{m} N$ and $T_{m}\left(Z^{-1}(z)\right)$ to the submanifold $N$ and to the common level surface $Z^{-1}(z) \subset M$ of functions in $Z$, respectively, intersect in general position, that is, transversally; here $z=Z(m)$.

Remark 3.2.4. The fact that the foliation $\Xi_{N}(Z)$ is pseudo-Hamiltonian may not imply that it is $\left(J_{N} d Z\right.$ )-Liouville (see $\S 2.1$ ), because although the vector fields $J d Z_{1}, \ldots, J d Z_{k}$ define a foliation on $N$, they may not be pairwise commutative on $N$. Nevertheless being pseudo-Hamiltonian is quite a strong hypothesis, since it means that on each $k$-dimensional leaf there exist $k$ vector fields that are tangent to this leaf and linearly independent at each point of it. This condition is a key one if the leaf is compact; for example, it 
imposes strong restrictions on the topology of such a leaf, but the leaf no longer has to be diffeomorphic to the torus as in the Liouville case.

Suppose that in addition we have a system with Hamiltonian $H$ such that the following two conditions hold:

e) (Criticality of the set $(H, Z)$ or sliding) At each point $m \in N$ the differential of the function $H$ regarded as a function on the whole of $M$ is a linear combination of the differentials of the functions in the set $Z: d H=\sum_{i=1}^{k} \lambda_{i} d Z_{i}$, where $\lambda_{i}=\lambda_{i}(m) \in \mathbf{R}$.

f) ( $Z$-symmetry) The vector field $J d H$ commutes on $N$ with each field $J d Z_{i}$ :

$$
\left.\left[J d H, J d Z_{i}\right]\right|_{N}=0, \quad i=1, \ldots, k .
$$

Definition 3.2.4. In this case and under the condition that the set of functions $Z$ defines a Hamiltonian foliation $\Xi_{N}(Z)$ on $N$ we say that the system has the set $Z$ of Hamiltonian-sliding symmetries or integrals on $N \subseteq M$. One may also say that the system is locally Hamiltonian-Z-integrable on $N$. If $\Xi_{N}(Z)$ is, in fact, a fibration (toric fibration), then the system is said to be Hamiltonian- (Hamiltonian-torically) $Z$-integrable on $N$. If in addition $N=M$, then it is said to be completely integrable (torically completely integrable).

If $\Xi_{N}(Z)$ is not a Hamiltonian but merely a weakly Hamiltonian foliation, fibration, or a toric fibration, then in the above definitions the term "Hamiltonian-" must be replaced by "weakly Hamiltonian-". If $\Xi_{N}(Z)$ is pseudo-Hamiltonian, then we say that the system has pseudo-Hamiltonian-sliding symmetries on $N$; these symmetries are not in general pairwise commutative on $N$. When $\Xi_{N}(Z)$ is a fibration (a toric fibration), the system is said to be $Z$-pseudo-integrable (torically $Z$-pseudo-integrable) on $N$.

Sometimes Hamiltonian foliations, fibrations, toric fibrations, and the corresponding integrable systems are called strongly Hamiltonian in order to better distinguish them from weakly Hamiltonian ones.

Remark 3.2.5. For complete compatibility with the definitions of $\S 2$ we must speak about abelian symmetries. But as our terminology is already cumbersome we shall omit the word "abelian". In fact, nonabelian symmetries in the Hamiltonian case are not considered explicitly in the paper. To be precise, they are investigated in $\S 7$ : in the terminology used there, such symmetries are "Lie groups with Poisson action" preserving the Hamiltonian $H$ of the system; see $\S \S 7.1-7.3$.

Remark 3.2.6. Actually, for "genuine" global integrability on $N \subseteq M$ we also need the submanifold $N$ to be embedded in $M$ or at least the fibres $\Lambda$ of the fibration $\Xi_{N}(Z)$ be embedded in $M$; see the definition at the beginning of $\S 2.1$. If $N$ is embedded in $M$, then the fibres $\Lambda$ that form a fibration of $N$ are embedded in $M$. But if a fibre $\Lambda$ is not embedded in $M$, then it appears that the behaviour of the solutions of the system on a typical submanifold $\Lambda$ will in practice be the same as the behaviour of the solutions of nonintegrable systems.

Indeed, although the behaviour of solutions on a nonembedded $\Lambda$ will be fine, for $\operatorname{dim} \Lambda<\operatorname{dim} M, \Lambda$ itself may be everywhere dense in the whole of $M$. As a result the behaviour of solutions on such a fibre of an "integrable" submanifold $N \subset M$ may, in all probability, be quite chaotic with respect to the entire phase space $M$. However, in the case of toric integrability on $N$ the fibres $\Lambda$, being tori, will always be embedded in $M$. They will be invariant tori of the system with conditionally periodic motion on them even if the submanifold $N$ is not embedded.

Nevertheless, for the sake of generality neither here nor in $\S 2$ have we imposed the condition that $N$ or the fibres $\Lambda$ are embedded in $M$. The fact is that this restriction is purely "ideological" and in particular, it would have no effect on the arguments or the 
assertions in this paper. But we shall bear in mind that for the solutions to have regular behaviour, which is inherent in solutions of systems integrable in the standard sense on the entire phase space, one must require that either $N$ or the fibres $\Lambda$ be embedded in $M$.

Remark 3.2.7. In the whole set of Definitions 3.2.4, except for the last definitions of systems with pseudo-Hamiltonian sliding symmetries and pseudo-integrable systems, we can take $H$ to be one of the functions $Z_{i}, i=1, \ldots, k$, in the set $Z$ defining the corresponding foliation or fibration $\Xi_{N}(Z)$. Then both additional conditions e) (the sliding condition) and $\mathrm{f})(Z$-symmetry) are redundant, since they are satisfied automatically. However, in the last definitions, that is, the ones related to local and global pseudo-integrability, only condition e) will be automatically satisfied for $H=Z_{i}$.

3.3. Systems with sliding symmetries. Relations between various properties of sets of functions $Z$ and the foliations defined by these sets. In Remarks 3.2.1-3.2.4 and 3.2.7 we mentioned some relations between the definitions stated in $\S 3.2$ and $\S 2$ and considered the properties of the objects defined there in some special cases. In $\S 3.3$ we state several more assertions of the same type related to these definitions. The proofs are simple and will all be given together in $\S 3.5$, at the end of this section. In all these assertions, unless we stipulate otherwise, by $N \subseteq M$ we mean an arbitrary submanifold of a symplectic manifold $\left(M, \omega^{2}\right)$ and denote by an upper tilde the restriction to $N$ of a given function defined on the whole of $M$.

The following proposition justifies the use of the term "integral" instead of "symmetry" in Definition 3.2.4. It also contains the assertion that weakly Hamiltonian-integrable systems on $N \subseteq M$ are Liouville-integrable on $N$, that is, they are integrable in the sense of $\S 2$.

Proposition 3.3.1. Suppose a system with Hamiltonian $H$ has a set $Z=\left(Z_{1}, \ldots, Z_{k}\right)$ of pseudo-Hamiltonian-sliding symmetries on a submanifold $N \subseteq M$. Then this submanifold is invariant under the local phase flow of the system, and the restrictions $\tilde{Z}_{i}:=\left.Z_{i}\right|_{N}$ are integrals of the system restricted to $N$, that is,

$$
J_{m} d H \in T_{m} N, \quad d Z_{i}\left(J_{m} d H\right)=0 \quad \text { for all } i=1, \ldots, k, m \in N .
$$

Suppose that $Z=\left(Z_{1}, \ldots, Z_{k}\right)$ is a set of not merely pseudo-Hamiltonian-sliding but weakly Hamiltonian-sliding symmetries on $N \subseteq M$. Set $J_{N} d H:=\left.(J d H)\right|_{N}$. Then the restricted vector fields $J_{N} d Z_{1}, \ldots, J_{N} d Z_{k}$ form an abelian set of sliding symmetries of the vector field $\tilde{V}:=J_{N} d H$ regarded as a field of general form, that is, in the sense of Definition 2.1.3.

In the case where the foliation $\Xi_{N}(Z)$ is a fibration (a toric fibration) the vector field $\tilde{V}$ is A-integrable (torically A-integrable) on $N$ in the sense of Definitions 2.1.3 and 2.2.1, where $A:=J_{N} d Z$. In particular, in the case of toric weakly Hamiltonian integrability on $N$ the motion of the system on the integrability submanifold $N$ is conditionally periodic on the invariant tori $\Lambda$ that are the fibres of the fibration $\Xi_{N}(Z)$; see Proposition 2.2.1.

In the following proposition we state a certain sufficient condition for Hamiltonian vector fields to define a Liouville foliation on a submanifold $N \subseteq M$ in the sense of $\S 2$.

Proposition 3.3.2. Let $Z=\left(Z_{1}, \ldots, Z_{k}\right)$ be a set of functions on $\left(M, \omega^{2}\right)$, and let $N \subseteq M$ be an arbitrary submanifold. Suppose that the vector fields $J d Z_{1}, \ldots, J d Z_{k}$ are tangent to $N$ and linearly independent at each point $m \in N$. Then these vector fields commute pairwise on $N$ and therefore define a Liouville foliation on $N$ if and only if $\left.\left(d\left\{Z_{i}, Z_{j}\right\}\right)\right|_{N}=0$ for all $i, j=1, \ldots, k$.

Proposition 3.3.3 asserts that the isotropy of the foliation defined on $N \subseteq M$ by Hamiltonian vector fields is equivalent to the fact that the Hamiltonians corresponding to these fields are pairwise in involution on $N$. 
Proposition 3.3.3. Suppose that $Z_{1}, \ldots, Z_{k}$ are some functions on a symplectic manifold $M$ such that the linear spans $\mathcal{J}_{m} d Z$ of the vectors of the fields $J d Z_{1}, \ldots, J d Z_{k}$ define a Frobenius integrable distribution on some submanifold $N \subseteq M$ that is $k$-dimensional at each point, that is, a foliation of $N$. Then this foliation is isotropic if and only if $\left.\left\{Z_{i}, Z_{j}\right\}\right|_{N}=0$ for all $i, j=1, \ldots, k$, or the equivalent equalities $\left.d Z_{i}\left(J d Z_{j}\right)\right|_{N}=0$ hold for the same $i, j$.

The following Proposition 3.3.4 together with its Corollary 3.3.1 show that in the special case of a symplectic submanifold $N \subseteq M$, verification of the conditions in Definition 3.2.2 of a Hamiltonian foliation simplifies substantially. Namely, condition b) that $\tilde{Z}$ is regular and condition c) that the fields $J d Z_{1}, \ldots, J d Z_{k}$ are pairwise commutative follow from the remaining conditions a) that $N$ is invariant and d) that the leaves are isotropic and $\mathrm{b}^{\prime}$ ) that $Z$ is regular on $N$. Here d) can be replaced by the involutivity condition $\left.\{Z, Z\}\right|_{N}=0$ on $N$. Thus, to establish the existence of the Hamiltonian foliation $\Xi_{N}(Z)$ there is no need to verify the Frobenius integrability of the distribution $\mathcal{J}_{N} d Z$ or the existence of the leaves $\Lambda$.

In addition, verifying the existence of Hamiltonian-sliding symmetries (see Definition 3.2.4) for the system also simplifies, since in this case condition $\mathrm{f}$ ) that the field $J d H$ commutes with the fields $J d Z_{1}, \ldots, J d Z_{k}$ follows from the other conditions, that is, from conditions a), $\left.b^{\prime}\right), d$ ), and e). In particular, it is redundant to verify both commuting conditions in this definition, that is, c) and $\mathrm{f}$ ).

Proposition 3.3.4. Let $N \subseteq M$ be a symplectic submanifold. Then the following three assertions hold.

A) Suppose that $Z=\left(Z_{1}, \ldots, Z_{k}\right)$ is a set of functions on $M$ such that $Z$ and $N$ satisfy only the following three conditions. The differentials $d Z_{1}, \ldots, d Z_{k}$ of these functions are linearly independent at each point $m \in N$, while $\left.\left\{Z_{i}, Z_{j}\right\}\right|_{N}=0$ for $i, j=1, \ldots, k$ and the vector fields $J d Z_{1}, \ldots, J d Z_{k}$ are tangent to the submanifold $N$. Then the distribution $\mathcal{J}_{N} d Z$ defines a Hamiltonian foliation $\Xi=\Xi_{N}(Z)$ on $N$. This foliation $\Xi_{N}(Z)$ coincides with the Hamiltonian foliation $\Xi(\tilde{Z})$ of the symplectic manifold $\left(N, \tilde{\omega}^{2}\right), \tilde{\omega}^{2}:=\left.\omega^{2}\right|_{N}$, defined by the set $\tilde{Z}=\left(\tilde{Z}_{1}, \ldots, \tilde{Z}_{k}\right)$ of functions on $N$, where $\tilde{Z}_{i}:=\left.Z_{i}\right|_{N}$.

The converse assertion is of course also true, namely, that if the foliation $\Xi_{N}(Z)$ is Hamiltonian on any submanifold $N \subseteq M$, then conditions a), $\left.\mathrm{b}^{\prime}\right)$ and d) of Definition 3.2.2 hold.

B) Suppose that a system with Hamiltonian $H$ has some set of Hamiltonian-sliding symmetries $Z$ on a submanifold $N \subseteq M$; see Definition 3.2.4. Then the vector field $J d H$ is tangent to $N$. Furthermore, its restriction to $N$ is a Hamiltonian vector field on the symplectic manifold $\left(N, \tilde{\omega}^{2}\right)$ with the Hamiltonian function $\tilde{H}=\left.H\right|_{N}$ and with the set $\tilde{Z}$ of Hamiltonian-sliding symmetries on the whole of $N$.

C) In this situation, in Definition 3.2 .4 of a system with a set of Hamiltoniansliding symmetries $Z$ on $N \subseteq M$ condition $\mathrm{f}$ ) (of $Z$-symmetry of the system) can be dropped, since it follows from the other conditions of this definition, that is, from conditions a), b), c), d) and e).

Corollary 3.3.1. In the case of a symplectic submanifold $N \subseteq M$ all three conditions of pseudo-Hamiltonian, weakly Hamiltonian, and simply Hamiltonian foliation $\Xi=\Xi_{N}(Z)$ of the submanifold $N$ are equivalent.

Moreover, for arbitrary functions $Z_{i}: M \rightarrow \mathbf{R}, i=1, \ldots, k$, to define a Hamiltonian foliation $\Xi_{N}(Z)$ on $N$ it is sufficient that the vector fields $J d Z_{1}, \ldots, J d Z_{k}$ simply define on $N$ a field of $k$-dimensional isotropic planes tangent to $N$. 
Suppose in addition that we have a system, defined on the whole of $M$, for which the vectors of its field $J d H$ are contained in the planes $\mathcal{J}_{m} d Z$ of this distribution for all points $m$ of the symplectic submanifold $N \subseteq M$, that is, $J_{m} d H \in \mathcal{J}_{m} d Z$ for all $m \in N$. Then this system is locally Hamiltonian-Z-integrable on $N$. If the foliation $\Xi_{N}(Z)$ is a fibration (a fibration with compact fibres), then the system is Hamiltonian(Hamiltonian-torically) Z-integrable on $N$.

In particular, for a foliation of a symplectic submanifold to be Hamiltonian it is sufficient that this foliation be defined by Hamiltonian vector fields and be isotropic. Note that the isotropy of the leaves alone is not enough for the foliation to be Hamiltonian. Moreover, there exist isotropic foliations to which no Hamiltonian systems correspond in the sense that the leaves of the foliation cannot be the invariant surfaces of such a system. This is shown by the following example; see 14. Consider a field of hyperplanes on a symplectic manifold $M$ that is not Frobenius-integrable. The Hamilton operator $J$ defines the dual field of straight lines on $M$. The integral curves of this field define a one-dimensional foliation of $M$, which is isotropic, since any curve is isotropic. But these curves obviously cannot be the trajectories of any Hamiltonian system and therefore the foliation into these curves cannot be Hamiltonian.

3.4. Properties of strongly Hamiltonian foliations and fibrations. It is clear that in the general situation verifying that a system is local integrable on an arbitrary submanifold $N \subseteq M$, that is, one that is not necessarily symplectic, is much harder than proving local Hamiltonian integrability on a symplectic submanifold $N$; see Corollary 3.3.1. The main result of this section, Proposition 3.4.1 (see also its Corollaries 3.4.1 and 3.4.2) shows, in particular, that proving local Hamiltonian $Z$-integrability on an arbitrary $N$ is not much more difficult than in the case of symplectic $N$. One must merely verify that $\tilde{Z}=\left.Z\right|_{N}$ is regular and then verify the same conditions as in the case of symplectic $N$ (that is, conditions a), d), and e) of Definition 3.2.4) and in addition verify condition $c^{\prime}$ ) that the distribution $\mathcal{J}_{N} d Z$ is regular.

Thus, by comparison with the case of a symplectic submanifold $N$ one must in addition verify conditions b) and $\mathrm{c}^{\prime}$ ) of Definition 3.2.4. But while the first is important, its verification is relatively simple; proving $\mathrm{c}^{\prime}$ ), that the distribution $\mathcal{J}_{N} d Z$ is integrable, based on the Frobenius conditions (10), is much harder. We could not get rid of this condition and it is unclear whether this is ever possible.

However, in Definition 3.2.4, where we define globally Hamiltonian-integrable systems, condition $\mathrm{c}^{\prime}$ ) follows from the other conditions, roughly speaking, from the existence of a fibration of $N$ (see Remark 3.2.1) and it is global integrability that is of interest in applications. (Nevertheless, this question of whether the distribution $\mathcal{J}_{N} d Z$ is integrable does not seem to be without any significance.) We emphasize that, just as for symplectic $N$, both commutativity conditions c) and f) involved in this definition are lifted in the situation of foliations; more precisely, for foliations, c) is replaced by $\mathrm{c}^{\prime}$ ). Note that the proofs of all these facts are much more cumbersome than when $N$ is symplectic.

We emphasize that in Definition 3.2.4 for weakly Hamiltonian-integrable systems in any situation - of foliations, fibrations, or toric fibrations - we could not manage to derive the commutativity conditions c) and f) from the other conditions. Thus, the importance of $\left.Z\right|_{N}$ being regular is again highlighted.

Proposition 3.4.1 will also be used in the proof of Theorem 5.5.1 B), the main generalization of Gordon's theorem. Its statement contains several conditions equivalent to the existence of a Hamiltonian foliation of a submanifold $N \subseteq M$. To formulate them we shall need the following notions of "nondegenerate isotropy" and being "quasi-Lagrangian" for a foliation of $N$ into isotropic leaves. 
Let $N$ be an arbitrary submanifold of a symplectic manifold $\left(M, \omega^{2}\right)$, and $Q \subseteq N$ an arbitrary submanifold of it that is isotropic in $M$, that is, $\left.\omega^{2}\right|_{Q}=0$. Let $K_{m}=K_{m}^{N}$ denote the kernel of the restriction $\left.\omega^{2}\right|_{T_{m} N}$ of the 2 -form $\omega^{2}$ to the tangent space $T_{m} N$ to $N$ at a point $m \in N$, that is,

$$
K_{m}:=\left\{\xi \in T_{m} N \mid \omega^{2}(\xi, \eta)=0 \quad \forall \eta \in T_{m} N\right\} .
$$

In other words, $K_{m}=T_{m} N \cap\left(T_{m} N\right)^{\perp}$, where $\left(T_{m} N\right)^{\perp}$ is the skew-orthogonal complement of $T_{m} N$ in the symplectic linear space $\left(T_{m} M,\left.\omega^{2}\right|_{T_{m} M}\right)$.

Definition 3.4.1. We say that the isotropic submanifold $Q \subseteq N \subseteq M$ is nondegenerateisotropic in $N$ at a point $m \in Q$ if the tangent space $T_{m} Q$ to $Q$ at the point $m$ intersects $K_{m}$ only in the zero vector: $T_{m} Q \cap K_{m}=\{0\}$. We say that the whole of $Q$ is nondegenerate-isotropic in $N$ if this is true at each point $m \in Q$. An isotropic foliation of the submanifold $N \subseteq M$ is said to be nondegenerate-isotropic if each of its leaves $\Lambda$ is nondegenerate-isotropic in $N$.

Remark 3.4.1. Each isotropic submanifold $Q$ of a symplectic submanifold $N \subseteq M$ is nondegenerate-isotropic in $N$. On the other hand, if $N$ is itself isotropic, then any submanifold $P \subseteq N$ is not nondegenerate-isotropic in $N$ at any point $m \in P$.

We now define what we mean by quasi-Lagrangian. Let $\Xi$ be some isotropic $k$-dimensional foliation of an arbitrary submanifold $N \subseteq M$ having the following properties. For each point $m_{0} \in N$ there exists a $2 k$-dimensional symplectic submanifold $S \subseteq N$ containing the point $m_{0}$ and composed of pieces of the leaves $\Lambda$ of the foliation $\Xi$. More precisely, for any point $m \in S, T_{m} \Lambda_{m} \subset T_{m} S$, where $\Lambda_{m} \ni m$ is a leaf of the foliation $\Xi$, and on the left and on the right there are tangent spaces at $m$ to $\Lambda_{m}$ and $S$, respectively. In particular, we must have $m_{0} \in \Lambda_{m_{0}} \cap S \subset S \subseteq N \subseteq M$.

Definition 3.4.2. In this case the isotropic foliation $\Xi$ of the submanifold $N \subseteq M$ is said to be quasi-Lagrangian.

This term is used in connection with the fact that the leaves of the restriction of this foliation to $S$ are Lagrangian submanifolds of the symplectic manifold $\left(S,\left.\omega^{2}\right|_{S}\right)$.

Proposition 3.4.1. A) Suppose that a set $Z=\left(Z_{1}, \ldots, Z_{k}\right)$ of functions on $M$ defines a pseudo-Hamiltonian foliation $\Xi=\Xi_{N}(Z)$ on an arbitrary submanifold $N \subseteq M$. In other words, suppose the vector fields $J d Z_{1}, \ldots, J d Z_{k}$ are tangent to the submanifold $N$ and the linear spans $\mathcal{J}_{m} d Z$ of the vectors of these fields at points $m \in N$ define a $k$-dimensional isotropic foliation on $N$. Then the following four conditions are equivalent:

a) the foliation $\Xi$ is nondegenerate-isotropic;

b) the foliation $\Xi$ is quasi-Lagrangian;

c) the set $\tilde{Z}=\left.Z\right|_{N}$ of the restrictions to $N$ of the functions in $Z$ is regular on $N$;

d) the foliation $\Xi$ is the Hamiltonian foliation defined by $Z$.

B) Consider Definition 3.2.4 of a system with a set $Z$ of Hamiltonian-sliding symmetries on $N \subseteq M$. Then in this definition, condition $\mathrm{f}$ ), $\left.[J d H, J d Z]\right|_{N}=0$ (the vector field $J d H$ of the system commutes on $N$ with the vector fields $\left.J d Z_{i}, i=1, \ldots, k\right)$, is redundant and can be omitted, since it always follows from conditions a)-e) in Definition 3.2.4.

The proof of Proposition 3.4.1 is rather cumbersome and will be given in $\S$ 6.2. Proposition 3.4.1 A) obviously implies the following.

Corollary 3.4.1. In Definition 3.2.2, when defining a Hamiltonian foliation $\Xi_{N}(Z)$ of a submanifold $N \subseteq M$, condition c) that the fields $J d Z_{1}, \ldots, J d Z_{k}$ be pairwise commutative can be replaced by the weaker condition $\mathrm{c}^{\prime}$ ) that the field of planes $\mathcal{J}_{N} d Z$ be integrable. 
Also, when defining a Hamiltonian fibration, condition c), as well as the weaker condition $\left.\mathrm{c}^{\prime}\right)$ can be removed altogether, since the condition for a fibration obviously implies the integrability of the field of planes $\mathcal{J}_{N} d Z$ on $N$; see Remark 3.2.1.

Remark 3.4.2. Let us compare the conditions that guarantee that a foliation of a submanifold $N \subseteq M$ is Hamiltonian, when $N$ is arbitrary (see Proposition 3.4.1 A) and Corollary 3.4.1) and when $N$ is symplectic (see Proposition 3.3.4A)). We see that if $N \subseteq M$ is symplectic it is easier to verify these conditions; cf. the beginning of this subsection. The main point is that in this special case the condition of Frobenius integrability is not imposed on the field of planes $\mathcal{J}_{N} d Z$ (see conditions c) and $\mathrm{c}^{\prime}$ ) of Definitions 3.2.2 and 3.2.3). This is because this condition follows from the involutivity of $Z$ on $N$, that is, from the condition $\left.\{Z, Z\}\right|_{N}=0$, which immediately implies that the fields $J d Z_{1}, \ldots, J d Z_{k}$ are pairwise commutative on $N$; see also the proof of Proposition 3.3.4 A) below. Furthermore, the equivalence of conditions a)-d) of Proposition 3.4.1 A) is no longer relevant, since if $N$ is symplectic any isotropic foliation is nondegenerate-isotropic; see Remark 3.4.1.

The three assertions given below are useful for finding the global and local integrability submanifolds of the phase spaces of concrete systems. The following is obvious.

Corollary 3.4.2. Suppose that the distribution $\mathcal{J}_{N} d Z$ defined on a submanifold $N \subseteq M$ by a set $Z=\left(Z_{1}, \ldots, Z_{k}\right)$ defines an isotropic fibration of this submanifold. Then for the the system on $N$ to be Hamiltonian-Z-integrable it is sufficient to verify $\mathrm{b})$ that $Z$ is internally regular and the sliding condition e); see Definition 3.2.2. These two conditions are also necessary for Hamiltonian $Z$-integrability on $N$.

The following two assertions show that if we have involutivity on the entire manifold $M$, rather than on a submanifold $N \subseteq M$, the situation becomes much simpler.

Corollary 3.4.3. Suppose that a system with Hamiltonian $H$ is defined on $M$ and there is a set $Z=\left(Z_{1}, \ldots, Z_{k}\right)$ of functions on $M$ that are pairwise in involution on the entire symplectic manifold $M$, that is, $\{Z, Z\}=0$. Then $Z$ is a set of Hamiltonian-sliding symmetries of the system on some submanifold $N \subseteq M$ (see Definition 3.2.4) if and only if the following three conditions are satisfied:

a) $d H(m)=\sum_{i=1}^{k} \lambda_{i}(m) d Z_{i}(m)$ at each point $m \in N$, that is, the differential of $H$ must be a linear combination of the differentials of the functions in $Z$;

b) the vector fields $J d Z_{1}, \ldots, J d Z_{k}$ are tangent to the surface $N$;

c) the set $\tilde{Z}=\left(\tilde{Z}_{1}, \ldots, \tilde{Z}_{k}\right)$ of the restrictions $\tilde{Z}_{i}=\left.Z_{i}\right|_{N}$ to $N$ of the functions in $Z$ is regular on the whole of $N$; that is, $\operatorname{rank} \tilde{Z}_{*}=k$, where $\tilde{Z}_{*}$ is the derivative of the map $\tilde{Z}: N \rightarrow \mathbf{R}^{k}$.

The following proposition is the Hamiltonian analogue of Proposition 2.3.1 in the special case of abelian symmetries.

Proposition 3.4.2. Suppose that both the functions in $Z=\left(Z_{1}, \ldots, Z_{k}\right)$ and the functions in the set $(H, Z)$ extended by $H$ are pairwise in involution on the entire symplectic manifold $M$, that is, $\{Z, Z\}=0$ and $\{H, Z\}=0$. Consider the submanifold $N \subseteq M$ specified in $M$ by condition a) of the preceding corollary, that the differential of the function $H$ is linearly dependent on the differentials of the functions in $Z$ at a point $m$, and suppose that $N$ is a smooth submanifold of $M$. Suppose also that $Z$ (the set $\tilde{Z}=\left.Z\right|_{N}$ ) is regular at all the points of this submanifold. Then the tangency condition $\mathrm{b}$ ) in that corollary, $J_{m} d Z_{i} \in T_{m} N, i=1, \ldots, k, m \in N$, is automatically satisfied. Moreover, $Z$ will be a set of weakly Hamiltonian- (respectively, Hamiltonian-) sliding symmetries of the system on $N$; see Definition 3.2.4. 
Thus, in this case, where $\{H, Z\}=0$ and $\{Z, Z\}=0$ on the whole of $M$, finding the maximal submanifold $N$ on which the system is locally weakly or strongly Hamiltonian$Z$-integrable becomes especially easy, since in practice it amounts to specifying $N$ in $M$ by the sliding condition, Corollary 3.4.3 a). Note that specifying the submanifold $N \subseteq M$ by the Hamiltonian function $H$ and the set $Z$ can be done using Lagrange multipliers.

Proof of Corollary 3.4.3. It is sufficient to show that because the integrals in $Z$ are in involution on the whole of $M$, conditions a)-c) in this corollary imply Definition 3.2.4 a)f). According to Proposition 3.4.1 B), f) follows from conditions a)-d). Condition c), $\left.[J d Z, J d Z]\right|_{N}=0$, follows since $\{Z, Z\}=0$ using (5). Conditions a), b), d), and e) are obtained trivially.

Proof of Proposition 3.4.2. First we observe that if $(H, Z)$ is in involution on the whole of $M$, then (5) implies that the vector fields $J d H, J d Z_{1}, \ldots, J d Z_{k}$ are pairwise commutative on $M$. Since $J_{m}$ is an isomorphism, we can apply Proposition 2.3.1 in a simpler form, because the algebra $\gamma$ whose linear basis is formed by the vector fields $J d Z_{1}, \ldots, J d Z_{k}$ is abelian. Proposition 2.3.1 implies that $N$ is invariant, but we give a separate proof of this fact based on Proposition 2.1.1.

Using this proposition, which discusses the existence of local normalizing coordinates, and the fact that the $k+1$ fields are pairwise commutative, as we showed above, we obtain the following. If some point $m \in M$ belongs to $N$, that is, $J_{m} d H=$ $\sum_{i=1}^{k} \lambda_{i} J_{m} d Z_{i}$, then this relation is preserved at all the points of the trajectory of each of the fields $J d H, J d Z_{1}, \ldots, J d Z_{k}$ passing through $m$, with exactly the same coefficients $\lambda_{i}$. This means that these $k+1$ trajectories lie on $N$ and, consequently, the vector fields $J d H, J d Z_{1}, \ldots, J d Z_{k}$ are indeed tangent to $N$ at any of its points $m$.

By construction, the sliding condition e) in Definition 3.2.4 holds on $N$. The fact that $Z$ is a set of weakly Hamiltonian-sliding symmetries of the system on $N$ in the case $\operatorname{rank} Z_{*}=k$, or Hamiltonian-sliding symmetries for $\operatorname{rank} \tilde{Z}_{*}=k$, follows easily from the facts obtained above, Definition 3.2.4, and formulae (4) and (5). This proves Proposition 3.4.2.

To conclude this subsection we prove the following, which may be regarded as a local analogue of the generalization of Gordon's theorem.

Proposition 3.4.3. Suppose that some system with Hamiltonian $H$ is locally Hamiltonian-Z-integrable on some submanifold $N \subseteq M$. Then the coefficients $\lambda_{i}$ in the sliding condition e), $d H=\sum_{i=1}^{k} \lambda_{i} d Z_{i}$ on $N$ (see Definition 3.2.4), only depend locally on $Z$ : $\lambda_{i}=\lambda_{i}\left(\left.Z\right|_{N}\right)$.

Proof. The sliding condition shows that $d\left(\left.H\right|_{N}\right)=\left.\sum_{i=1}^{k} \lambda_{i}\right|_{N} d\left(\left.Z_{i}\right|_{N}\right)$. This fact and the regularity of $\left.Z\right|_{N}$ imply that $\left.H\right|_{N}=f\left(\left.Z\right|_{N}\right)$ locally. Consequently, the differentials of these two functions are also equal. From this and the regularity of $\left.Z\right|_{N}$ we obtain $\left.\lambda_{i}\right|_{N}=g_{i}\left(\left.Z\right|_{N}\right)$, where $g_{i}=\partial_{i} f, i=1, \ldots, k$, and $\partial_{i} f$ is the derivative of the function $f$ with respect to the $i$ th variable. Proposition 3.4.3 is proved.

Note that the regularity of $\left.Z\right|_{N}$ is a crucial condition for this assertion to be true, since if it fails, then the coefficients $\lambda_{i}$ may be quite arbitrary. (For example, this will be the case for a function of the form $H=\alpha \cdot Z$ on the submanifold $N=\{Z=0\}$, where $\left.\alpha\right|_{N} \neq$ const.) The same is also true for the genuine, global generalizations of Gordon's theorem; see Propositions 5.1.1, 5.3.1 and Theorem 5.5.1 B).

\subsection{Proof of Propositions 3.3.1-3.3.4.}

Proof of Proposition 3.3.1. By Definition 3.2.3 a), defining a Hamiltonian foliation, the 
vector fields $J d Z_{1}, \ldots, J d Z_{k}$ are tangent to the submanifold $N \subseteq M$. As we remarked, $J_{m}: T_{m}^{*} M \rightarrow T_{m} M$ is a linear isomorphism (see $\S 3.1$ or [1); consequently, $J_{m} d H=$ $\sum_{i=1}^{k} \lambda_{i}(m) J_{m} d Z_{i}$. From this we obtain $J_{m} d H \in T_{m} N, m \in N$. Now (4) implies that

$$
d Z_{j}(J d H)=\omega^{2}\left(J d H, J d Z_{j}\right)=\sum_{i=1}^{k} \lambda_{i} \omega^{2}\left(J d Z_{i}, J d Z_{j}\right), \quad j=1, \ldots, k .
$$

The last sum vanishes on $N$, since $\omega^{2}\left(J d Z_{i}, J d Z_{j}\right)=0$ on $N$ for $i, j=1, \ldots, k$; this is because the pseudo-Hamiltonian foliation $\Xi_{N}(Z)$ is isotropic. The other assertions follow trivially from Definitions 3.2.4, 3.2.3, 2.1.3, and 2.2.1. Proposition 3.3.1 is proved.

Proposition 3.3.2 obviously follows from (5), which links the Lie and Poisson brackets. Proposition 3.3.3 is an obvious consequence of (4), which links the Poisson bracket with the symplectic structure $\omega^{2}$ on $M$, and the Poisson bracket with the derivative of a function along a Hamiltonian vector field.

Proof of Proposition 3.3.4 A). We need the following useful lemma.

Lemma 3.5.1. Let $F=\left(F_{1}, \ldots, F_{r}\right)$ be a set of functions defined on a symplectic manifold $M$ and suppose that $F$ is regular at every point of a symplectic submanifold $N \subseteq M$ such that the vector fields $J d F_{1}, \ldots, J d F_{r}$ are tangent to $N$; in other words, we require that $\operatorname{rank} Z_{*}(m)=k$ and $J_{m} d F_{i} \in T_{m} N, i=1, \ldots, k$, for all $m \in N$. Then the set of restrictions $\tilde{F}=\left(\tilde{F}_{1}, \ldots, \tilde{F}_{r}\right)$ of the functions in $F$ to $N$ is also regular on $N$. Furthermore, $\left.\left\{F_{i}, F_{j}\right\}\right|_{N}=\left\{\tilde{F}_{i}, \tilde{F}_{j}\right\}_{N}$, where $\{,\}_{N}$ is the Poisson bracket corresponding to the symplectic manifold $\left(N, \tilde{\omega}^{2}\right), \tilde{\omega}^{2}=\left.\omega\right|_{N}$.

Proof of Lemma 3.5.1. By Lemma 3.1.1 A), which discusses the restriction of a Hamiltonian vector field to an invariant symplectic submanifold, in the notation of this lemma $\left.\left(J d F_{i}\right)\right|_{N}=\tilde{J} d \tilde{F}_{i}, i=1, \ldots, r$. Since the operators $J_{m}$ and $\tilde{J}_{m}$ are isomorphisms, the regularity of $F$ implies that the vector fields $\tilde{J} d \tilde{F}_{i}, i=1, \ldots, r$, are linearly independent at each point $m \in N$ and therefore the differentials $d \tilde{F}_{1}, \ldots, d \tilde{F}_{r}$ are also linearly independent at each point $m \in N$, that is, the set $\tilde{F}$ is regular on $N$. The equality of the Poisson brackets follows from the same lemma, more precisely, from Lemma 3.1.1 B). Lemma 3.5.1 is proved.

This lemma implies that $\tilde{Z}$ is regular on $N$. Lemma 3.1.1 and formula (5) imply that the restrictions of the vector fields $J d Z_{1}, \ldots, J d Z_{k}$ to $N$ are pairwise commutative. Now using (44) we see that the foliation $\Xi_{N}(Z)$ defined on $N$ by the set $J_{N} d Z$ of these fields is isotropic. Consequently, the foliation $\Xi_{N}(Z)$ of $N \subseteq M$ satisfies Definition $3.2 .2 \mathrm{a}$ )-d), that is, it is indeed Hamiltonian. The fact that the foliations $\Xi_{N}(Z)$ and $\Xi(\tilde{Z})$ defined on $N$ coincide follows from Lemma 3.1.1 A) in an obvious way. Applying Lemma 3.1.1 A) again, the foliation $\Xi(\tilde{Z})$ is Hamiltionian because the foliation $\Xi_{N}(Z)$ is. The proof of Proposition 3.3.4 A) is complete.

Proof of Proposition 3.3.4B). The fact that the field $J d H$ is tangent to $N$ follows from Proposition 3.3.1. That the restriction of the system to the submanifold $N$ is Hamiltonian follows from Lemma 3.1.1. The existence of the set of Hamiltonian-sliding symmetries $\tilde{Z}$ for the system restricted to $N$ is a consequence of the fact that $\Xi(\tilde{Z})$ is Hamiltonian, as we have just shown, Lemma 3.1.1 and the fact that $Z$ satisfies Definitions 3.2.4 e) and $\mathrm{f}$ ).

Proof of Proposition 3.3.4 C). To derive Definition 3.2.4 f) from conditions a)-e) we observe that e) implies that locally $\tilde{H}=\tilde{H}(\tilde{Z})$. Since $\left.\{Z, Z\}\right|_{N}=0$, Lemma 3.1.1B) implies that $\{\tilde{Z}, \tilde{Z}\}_{N}=0$. This, with (4), means that $\{\tilde{H}, \tilde{Z}\}_{N}=0$. Now using (15), 
$[\tilde{J} d \tilde{H}, \tilde{J} d \tilde{Z}]=0$, where $\tilde{J}$ is the Hamiltonian operator corresponding to the symplectic manifold $\left(N, \tilde{\omega}^{2}\right)$. Using Lemma 3.1.1 A) we obtain $\left.[J d H, J d Z]\right|_{N}=0$, that is, condition f) holds. The proof of Proposition 3.3.4 is complete.

\section{Hamiltonian Fibrations And integRable Systems IN TERMS OF SETS OF FUNCTIONS}

In the preceding section the structures we studied were, for the most part foliations, and in the main we used a geometric viewpoint, namely, as partitions of the distribution defined by a set of Hamiltonian vector fields into integral surfaces. The present section, $\S 4$ is devoted exclusively to fibrations - in fact, Hamiltonian fibrations - and to the corresponding integrable systems. We shall study these objects mainly in terms of sets of functions. In the invariant language of the preceding section this means in practice that we will study the system in a neighbourhood of a fibre of a Hamiltonian fibration. Here, by contrast with the general non-Hamiltonian case, that is, the study of Liouville fibrations (see $\S 2$ ), the semilocal study of Hamiltonian fibrations can be completely reduced to the study of sets of functions.

There are two reasons for this. First, Hamiltonian vector fields are defined by their Hamiltonian functions. Second, we can define the map $\psi: N \rightarrow B$, which fibres $N$ by the inverse images of points in $B$ in a neighbourhood $U$ in $N$ of the inverse image $\psi^{-1}(b)$ of each point $b \in B$, by the set $F$ of functions on $U$ corresponding to some local coordinates $y$ in a neighbourhood of the point $b$ in $B$, that is, $F=y \circ \psi$. The last fact is of course valid for any fibration of any manifold $N$ defined by a map $\psi: N \rightarrow B$, that is, one does not have to assume that $N$ is a submanifold of a symplectic manifold.

In $\S 4.1$ we define and describe some types of sets of functions defined on the entire phase space $M$, which are useful in the study of integrability. In $\S 4.2$ we define "Hamiltonian-integrable" on $N$ sets of functions $\Phi=(Z, \mathcal{F})$ defining the fibration of the submanifold $N \subseteq M$ by the common level surfaces, as well as the systems corresponding to these sets that are Hamiltonian- $\Phi$-integrable on $N$. The functions in the subset $\mathcal{F}$ may be defined only on $N$. We also explain the equivalence of the definitions of these two subsections in the case $N=M$.

In $\S 4.3$ we state Proposition 4.3.1, which asserts the equivalence of the definitions of Hamiltonian integrability in $\S 4.2$ given in terms of the common surfaces of sets of functions, and the corresponding invariant definitions in $\S 3.2$ based on Hamiltonian fibrations. We also state Proposition 4.3.2, which asserts the equivalence of several different definitions of Hamiltonian fibrations defined as common surfaces. This proposition may be regarded as a special case of Proposition 3.4.1 A) for the situation of fibrations; it reformulates it in terms of sets of functions.

4.1. Centred, involutive, and integrable sets of functions on a symplectic manifold. In this subsection we consider some types of sets of functions defined on the entire symplectic manifold $\left(M, \omega^{2}\right)$.

Let $F=\left(F_{1}, \ldots, F_{r}\right)$ and $G=\left(G_{1}, \ldots, G_{s}\right)$ be two sets of functions on $M, F_{i}: M \rightarrow$ $\mathbf{R}, G_{j}: M \rightarrow \mathbf{R}$. We denote by $\{F, G\}$ the rectangular matrix of size $r \times s$ composed of the pairwise Poisson brackets of the functions in these sets:

$$
\{F, G\}:=\left(\left\{F_{i}, G_{j}\right\}, i=1, \ldots, r, j=1, \ldots, s\right) .
$$

Definition 4.1.1. We call the matrix $\{F, G\}$ the Poisson matrix of the sets of functions $F$ and $G$.

When we used the shorthand $\{F, G\}=0$ above we meant that the Poisson matrix $\{F, G\}$ is equal to zero. We only need nonzero Poisson matrices in $\S 7$. 
Remark 4.1.0. This remark relates to the condition $\{Z, Z\}=0$ that the functions in a set $Z$ are in pairwise involution in the entire phase space $M$ and concerns all the propositions, both those given above and those stated below, of the following kind. In these propositions the condition $\{Z, Z\}=0$ is imposed in the whole of $M$, while by hypothesis a submanifold $N \subseteq M$ is given, and certain assertions are made on the behaviour of the Hamiltonian system only on $N$, or the weak or strong Hamiltonian $Z$-integrability of the system is asserted also only on $N$ (see Definitions 3.2.4). These propositions do not include Proposition 3.4.2 and the assertions in $\S 7$, since in them $N$ is to be found, rather than being fixed.

It is easy to see that the condition $\{Z, Z\}=0$ on $M$ can be replaced in these assertions and in Definition 3.2.4 by the condition of pairwise involutivity in the "linear approximation" of the functions in the set $Z=\left(Z_{1}, \ldots, Z_{k}\right)$ in a neighbourhood of the submanifold $N \subseteq M$. This means that the functions in the set can be defined only in some neighbourhood of the submanifold $N$ and satisfy $\{Z, Z\}(m)=O\left(\rho^{2}\right)$ for each point $m \in N$. Here $\rho=\rho(m, N)$ is the distance from a point $m$ to $N$ corresponding to some Riemannian metric defined in a neighbourhood of $N$ in $M$.

The fact is that in the proofs of all the assertions of this type the condition $\{Z, Z\}=$ 0 on $M$ is really only necessary for the following reason. From this condition, using formula (5) we find that the vector fields $J d Z_{1}, \ldots, J d Z_{k}$, which are assumed to be tangent to $N$, are pairwise commutative on $N$, that is, the corresponding Poisson brackets vanish on $N:\left.[J d Z, J d Z]\right|_{N}=0$. But this equality also follows from the weaker condition $\{Z, Z\}(m)=O\left(\rho^{2}\right)$. We require that equalities of the type $\{Z, Z\}=0$ hold on the whole of $M$ mainly so that the statements can be concise.

Furthermore, in this subsection we consider only the phase space $M$ itself. Let $F=(Z, \mathcal{G})$ be a set of functions on $M$ divided into two subsets: $F=(Z, \mathcal{G})=$ $\left(Z_{1}, \ldots, Z_{k}, \mathcal{G}_{1}, \ldots, \mathcal{G}_{l}\right)$, where $k \geq 1$ and $l \geq 0$.

Definition 4.1.2. A set $F=(Z, \mathcal{G})$ of functions on $M$ that is regular on $M$ is called centred if all the functions in the subset $Z$ are pairwise in involution with all the functions in $F$, that is,

$$
\operatorname{rank} F_{*}=k+l \quad \text { and } \quad\{Z, F\}=0 \quad \text { on } \quad M .
$$

In this case the subset $Z$ is called a distinguished central subset of the set $F=(Z, \mathcal{G})$.

If $l=0$, then such a set $F$ is called involutive, and if $2 k+l=2 n$, where $2 n=\operatorname{dim} M$, then it is called integrable on $M$.

Remark 4.1.1. In the definition of a centred set it is not necessary that the second subset $\mathcal{G}$ contains no functions in involution with all the functions in the set $F$. In other words, $Z$ does not have to be a maximal subset of the set $F$ such that $\{Z, F\}=0$ but merely some subset of this type.

We also note that if $F=(Z, \mathcal{G})$ is integrable, the subset $Z$ will automatically be a maximal subset of this type. This follows easily from the next lemma, which is a trivial consequence of the nondegeneracy of the 2-form $\omega^{2}$ defining the symplectic structure on $M=M^{2 n}$; see [5].

Lemma 4.1.1. Let $F=\left(F_{1}, \ldots, F_{r}\right)$ and $G=\left(G_{1}, \ldots, G_{s}\right)$ be two arbitrary regular sets of functions on a symplectic manifold $\left(M^{2 n}, \omega^{2}\right)$ such that $\{F, G\}=0$. Then $r+s \leq 2 n$, where $2 n=\operatorname{dim} M^{2 n}$.

Remark 4.1.2. This lemma implies that if $F=(Z, \mathcal{G})$ is a centred set of functions on $M^{2 n}$, where $Z=\left(Z_{1}, \ldots, Z_{k}\right), \mathcal{G}=\left(\mathcal{G}_{1}, \ldots, \mathcal{G}_{l}\right)$, then $2 k+l \leq 2 n$. Thus, the integrable case is a limiting one. 
Definition 4.1.3. Suppose that a system with Hamiltonian $H$ is defined on $M$ having a set of integrals $F=(Z, \mathcal{G})$ integrable on $M$, that is, such that $\{H, F\}=0$. Then we say that such a system is completely integrable by the set of integrals $F$, or $F$-integrable on $M$, for short.

4.2. Systems and sets of functions Hamiltonian-integrable on a submanifold. In $\S 4.3$ we state Propositions 4.3.1 A) and 4.3.1 B), which show, in particular, the following. The definitions of integrable sets of functions and of integrable systems on $M$ given in $\S 4.1$ are compatible with Definition 3.2.2 of Hamiltonian fibrations of a submanifold $N \subseteq M$ and with Definition 3.2.4 of systems Hamiltonian-integrable on $N$ in the case $N=M$.

For fibrations and sets of functions to be compatible when $N \subseteq M$ is an arbitrary submanifold, we must consider mixed sets of functions. By this we mean sets of the form $\Phi=(Z, \mathcal{F})$, where $Z=\left(Z_{1}, \ldots, Z_{k}\right)$ is a set of functions defined on the whole of $M$, as above, and $\mathcal{F}=\left(\mathcal{F}_{1}, \ldots, \mathcal{F}_{l}\right)$ is a set of functions defined only on $N$.

Suppose that a mixed set $\Phi=(Z, \mathcal{F})$ satisfies the following five conditions:

a) $2 k+l=\operatorname{dim} N$

b) $\left.\{Z, Z\}\right|_{N}=0$;

c) the Hamiltonian vector fields defined by the functions in the central subset $Z$ are tangent to the submanifold $N \subseteq M$, that is,

$$
J_{m} d Z_{i} \in T_{m} N \quad \forall i=1, \ldots, k, \quad m \in N
$$

d) the functions in $\mathcal{F}$ are integrals of the restrictions of these fields to $N$, that is, on $N$ we have

$$
d \mathcal{F}_{j}\left(J d Z_{i}\right)=0, \quad i=1, \ldots, k, \quad j=1, \ldots, l ;
$$

e) let $F=(\tilde{Z}, \mathcal{F})$ denote the set of $k+l$ functions defined on $N$, where, as in $\S 3$, $\tilde{Z}=\left(\tilde{Z}_{1}, \ldots, \tilde{Z}_{k}\right), \tilde{Z}_{i}=\left.Z_{i}\right|_{N}$; then $F$ is regular on $N$.

Definition 4.2.1. If conditions a)-e) are satisfied, then the mixed set $\Phi=(Z, \mathcal{F})$ consisting of $k$ functions defined on $M$ and $l$ functions defined on $N \subseteq M$ is said to be Hamiltonian-integrable on the submanifold $N \subseteq M$. The subset $Z$ is called a central subset of the set $\Phi$, and the set $F=(\tilde{Z}, \mathcal{F})$ of functions on $N$ is said to be associated with $\Phi$.

If in addition all the connected components of all the common level surfaces $F^{-1}(y)$, $y \in \mathbf{R}^{k+l}$, of the functions in the set $F$ are compact, then we say that the set $\Phi$ is Hamiltonian-torically integrable on $N$.

Remark 4.2.1. The use of the term "torically" is related to the fact that all these components are tori, which form a locally trivial fibration of $N$. This fact will follow from the fact that this fibration is Hamiltonian in the sense of Definition 3.2.2, which we prove below (see Proposition 4.3.1 A)) and therefore it is Liouville, as well as from Proposition 2.2.1 (or see Proposition 3.3.1).

Suppose that a system with Hamiltonian $H$ is defined on the whole of $M$ and there is a mixed set of functions $\Phi=(Z, \mathcal{F})$ Hamiltonian- (Hamiltonian-torically) integrable on a submanifold $N \subseteq M$ and having the following property. At each point $m \in N$, if $d H$ and $d Z_{i}$ are the differentials of the functions $H$ and $Z_{i}$ respectively, regarded as functions on the whole of $M$, then $d H$ is linearly dependent on the $d Z_{i}: d H=\sum_{i=1}^{k} \lambda_{i} d Z_{i}$, where $\lambda_{i}=\lambda_{i}(m)$.

Definition 4.2.2. In this case we say that the system is Hamiltonian- (Hamiltoniantorically) $\Phi$-integrable on the submanifold $N \subseteq M$. 
Remark 4.2.2. When $N=M$ a mixed set $\Phi=(Z, \mathcal{F})=F$ can be regarded as a set of functions on $M$. Then if $\Phi$ is Hamiltonian-integrable on $N=M$ in the sense of Definition 4.2.1 this set will obviously be integrable on $M$ also in the sense of Definition 4.1.2. The converse assertion is also true, and the equivalence of Definitions 4.1.2 and 4.2.1 in the case $N=M$ easily follows from the comparison of the conditions imposed in them.

When $N=M$, Definition 4.2.2 is also fully equivalent to Definition 4.1.3 of a completely integrable system. These definitions differ, in fact, only in that the condition of being critical, $d H=\sum_{i=1}^{k} \lambda_{i} d Z_{i}$, is replaced by $\{H, F\}=0$ in Definition 4.1.3. Therefore their equivalence is an easy consequence of the following simple lemma.

Lemma 4.2.1. Suppose that a system with Hamiltonian $H$ is defined on $M$ and there is a set of functions $F=(Z, \mathcal{G})$ integrable on the whole of $M$, that is, such that $\{Z, F\}=0$, $\operatorname{rank} F_{*}=k+l$, and $2 k+l=2 n$, where $2 n=\operatorname{dim} M, Z=\left(Z_{1}, \ldots, Z_{k}\right), \mathcal{G}=\left(\mathcal{G}_{1}, \ldots, \mathcal{G}_{l}\right)$. Then $F$ is a set of integrals of the system if and only if $d H=\sum_{i=1}^{k} \lambda_{i} d Z_{i}$ at each point $m \in M$, where $\lambda_{i}=\lambda_{i}(m)$.

Proof. In one direction the assertion of the lemma follows from the nondegeneracy of the symplectic form $\omega^{2}$. In more detail, suppose that the system has such a set $F=(Z, \mathcal{G})$ of integrals, and suppose the opposite, that is, at some point $m \in M$ the differential $d H$ is not a linear combination of the differentials $d Z_{1}, \ldots, d Z_{k}$. Let $Z^{\prime}$ denote the set of $k^{\prime}=$ $k+1$ functions: $Z^{\prime}=\left(Z_{1}, \ldots, Z_{k}, H\right)$; then $\left\{Z^{\prime}, F\right\}=0$ and $k^{\prime}+k+l=2 k+l+1>2 n$, which contradicts Lemma 4.1.1.

Conversely, since the set $F=(Z, \mathcal{G})$ is Hamiltonian-integrable, we have $0=\left\{F_{j}, Z_{i}\right\}=$ $d F_{j}\left(J d Z_{i}\right), i=1, \ldots, k, j=1, \ldots, k+l$. Consequently,

$$
d F_{j}(J d H)=d F_{j}\left(J\left(\sum_{i=1}^{k} \lambda_{i} d Z_{i}\right)\right)=\sum_{i=1}^{k} \lambda_{i} d F_{j}\left(J d Z_{i}\right)=0,
$$

that is, all the functions in the set $F$ are indeed integrals of the system. Lemma 4.2.1 is proved.

4.3. Compatibility of the definitions for sets of functions and for fibrations. Conditions equivalent to the existence of the Hamiltonian fibration defined by a set of functions. The following proposition shows that Definition 4.2.1, which defines a set of functions $\Phi=(Z, \mathcal{F})$ Hamiltonian-integrable on an arbitrary submanifold $N \subseteq M$ agrees with Definition 3.2.2 of a Hamiltonian $Z$-fibration of the submanifold $N$, and in the case of a toric fibration these definitions in fact coincide.

Proposition 4.3.1. A) Suppose that $\Phi=(Z, \mathcal{F})$ is a set of functions Hamiltonianintegrable on a submanifold $N \subseteq M$, where $Z=\left(Z_{1}, \ldots, Z_{k}\right), \mathcal{F}=\left(\mathcal{F}_{1}, \ldots, \mathcal{F}_{l}\right)$, and let $F=(\tilde{Z}, \mathcal{F})$ be the set of functions on $N$ associated with $\Phi$. Then the central subset $Z$ of the set $\Phi$ defines a $k$-dimensional Hamiltonian fibration $\Xi_{N}(Z)$ of the submanifold $N$; see Definition 3.2.2. Here $F: N \rightarrow \mathbf{R}^{k+l}$ is the corresponding fibring map, that is, the fibres of the fibration $\Xi_{N}(Z)$ are the connected components of the inverse images $F^{-1}(y)$ of points $y \in \mathbf{R}^{k+l}$; see Definition 3.2.1.

Conversely, suppose that an involutive set $Z=\left(Z_{1}, \ldots, Z_{k}\right)$ of functions on $\left(M, \omega^{2}\right)$ defines a Hamiltonian fibration $\Xi=\Xi_{N}(Z)$ of a submanifold $N \subseteq M$ and let $\psi: N \rightarrow B$ be the corresponding fibring map. Then $\operatorname{dim} N=2 k+l$ and correspondingly $\operatorname{dim} B=k+l$, where $l \geq 0$; and if $l=0$, then $N$ is a symplectic submanifold of $M$.

Furthermore, let $K$ be any connected compact set contained in the inverse image $\psi^{-1}(b)$ of some point $b \in B$ under the map $\psi: K \subseteq \psi^{-1}(b) \subset N$. Then in some neighbourhood $U$ in $N$ of $K, K \subset U \subseteq N$, the converse assertion is also true. Namely, there exists a set of functions $\mathcal{F}=\left(\mathcal{F}_{1}, \ldots, \mathcal{F}_{l}\right)$ defined in $U$ such that the set $F=\left(\left.Z\right|_{U}, \mathcal{F}\right)$ 
defines the restriction of the map $\psi$ to $U$ in some local coordinates $y$ defined in a neighbourhood of the point $b \in B: y(\psi(m))=F(m)$ for all $m \in U$. Here the mixed set $\Phi=(Z, \mathcal{F})$ is Hamiltonian-integrable on $U$; see Definition 4.2.1.

A similar assertion is also valid for systems:

Proposition 4.3.1. B) Suppose that we have a system that is Hamiltonianly- $\Phi$-integrable on an arbitrary submanifold $N \subseteq M$ of a symplectic manifold $\left(M, \omega^{2}\right)$ in the sense of Definition 4.2.2, where $\Phi=(Z, \mathcal{F}), Z=\left(Z_{1}, \ldots, Z_{k}\right)$, and $\operatorname{dim} N=2 k+l$. Then the system is also Hamiltonian-Z-integrable on $N$ in the sense of Definition 3.2.4 with fibring map $F: N \rightarrow \mathbf{R}^{k+l}$, where $F$ is the set associated with $\Phi$, that is, $F=(\tilde{Z}, \mathcal{F})$ and $\tilde{Z}=\left.Z\right|_{N}$.

Conversely, suppose that we have a system that is Hamiltonian-Z-integrable on an arbitrary submanifold $N \subseteq M$ in the sense of Definition 3.2 .4 with fibring map $\psi$ : $N \rightarrow B$, where $Z=\left(Z_{1}, \ldots, Z_{k}\right)$ and $\operatorname{dim} B=k+l$. Then any connected compact set $K \subseteq \psi^{-1}(b) \subset N, b \in B$, has a neighbourhood $U$ in $N$ such that the system is Hamiltonian- $\Phi$-integrable on $U \subseteq N$ in the sense of Definition 4.2.2, where $\Phi$ is a mixed set with the following properties: it has the form $\Phi=(Z, \mathcal{F})$, where $\mathcal{F}$ is a set of $l$ functions on $U$ such that the set $F=\left(\left.Z\right|_{U}, \mathcal{F}\right)$ of functions on $U$ defines the restriction $\left.\psi\right|_{U}$ of the map $\psi$ to $U$ in some local coordinates $y$ defined in a neighbourhood of the point $b \in B$.

Remark 4.3.1. If the fibration $\Xi=\Xi_{N \psi}$ of the submanifold $N$ defined by the map $\psi$ is toric, then in both Propositions 4.3.1 A) and B) there is no need to consider a connected compact set $K \subseteq \Lambda$ instead of the fibre $\Lambda$ itself, since a torus is a compact set. So we find that if the fibres of the fibration are compact, Definitions 3.2.2 and 3.2.4 coincide with Definitions 4.2.1 and 4.2.2, respectively, at least semilocally, that is, in some neighbourhood of each fibre $\Lambda$ of this fibration. When a fibre $\Lambda \subseteq \psi^{-1}(b)$ is noncompact, the need for a compact set $K \subseteq \Lambda$ is related to the fact that it is easy to construct an example where for any neighbourhood $U$ of this fibre in $N$ there exist at least two connected components of the inverse image $\psi^{-1}\left(b^{\prime}\right)$ of one of the points $b^{\prime} \in B$ intersecting this neighbourhood. This nonconnectedness impedes the proof of Proposition 4.3.1A) and therefore, 4.3.1 B).

These two propositions have somewhat complex proofs and we shall give them in $\S 6.3$ together with the proof of the next proposition. It can be regarded as a reformulation of Proposition 3.4.1 A) in the special case when fibrations are defined by sets of functions, rather than by the integral surfaces of the distributions corresponding to vector fields.

Proposition 4.3.2. Let $N \subseteq M$ be an arbitrary submanifold of a symplectic manifold $\left(M, \omega^{2}\right)$, and suppose that $\bar{Z}=\left(Z_{1}, \ldots, Z_{k}\right)$ and $G=\left(G_{1}, \ldots, G_{r}\right)$ are sets of functions defined on $M$ and $N$, respectively, that are regular on $N: \operatorname{rank} Z_{*}(m)=k$ and $\operatorname{rank} G_{*}(m)=r$ at all the points $m \in N$. Suppose that these sets and $N$ satisfy all of the following four conditions:

$$
\left\{\begin{array}{l}
k+r=\operatorname{dim} N ;\left.\quad\{Z, Z\}\right|_{N}=0 ; \\
J_{m} d Z_{i} \in T_{m} N \quad \text { for all } i=1, \ldots, k, \quad m \in N ; \\
d G_{j}\left(J d Z_{i}\right)=0, \quad i=1, \ldots, k, \quad j=1, \ldots, r, \quad \text { on } N
\end{array}\right.
$$

(cf. conditions a)-d) of Definition 4.2.1). Then under these assumptions the following four conditions are pairwise equivalent.

a) Consider the fibration $\Xi=\Xi_{N G}$ of the submanifold $N$ into the connected components of the inverse images $G^{-1}(g), g \in \mathbf{R}^{r}$ of the map $G: N \rightarrow \mathbf{R}^{r}$; then this fibration, which is isotropic by the above conditions, is nondegenerate-isotropic. 
b) This isotropic fibration $\Xi_{N G}$ of the submanifold $N$ is quasi-Lagrangian.

c) The set $\tilde{Z}=\left.Z\right|_{N}$ of the restrictions to $N$ of the functions in the set $Z$ is regular on $N: \operatorname{rank} \tilde{Z}_{*}(m)=k$.

d) The set of functions $Z$ defines a Hamiltonian fibration $\Xi_{N}(Z)$ of the submanifold $N \subseteq M$ in the sense of Definition 3.2.2. In particular, this fibration defined by the vector fields $J d Z_{1}, \ldots, J d Z_{k}$ is Liouville. The fibration $\Xi_{N}(Z)$ coincides with the fibration $\Xi_{N G}$ corresponding to the map $G: N \rightarrow \mathbf{R}^{r}$ in a), in the sense that the fibres of these fibrations coincide.

Remark 4.3.2. It is easy to see that if $\Phi=(Z, \mathcal{F})$ is a mixed set of functions Hamiltonianintegrable on $N \subseteq M$ (see Definition 4.2.1), then the sets $Z$ and $G=\left(\left.Z\right|_{N}, \mathcal{F}\right.$ ) satisfy the main assumptions of Proposition 4.3.2 plus condition c) (that the set $\left.Z\right|_{N}$ is regular on $N$ ). Conversely, if the fibres of the fibration $\Xi=\Xi_{N G}$ are compact, then from Proposition 4.3.1 A) (see Remark 4.3.1), as $\Xi$ is assumed to be Hamiltonian in Proposition 4.3.2, we obtain the following fact. In a neighbourhood $U$ in $N$ of each such a fibre there exists a set of functions $\mathcal{F}$ such that the set $\Phi=(Z, \mathcal{F})$ is Hamiltonian-torically integrable on $U \subseteq N \subseteq M$ and fibres $U$ into the same fibres as the set $G$. In other words, the sets $\left.\Phi\right|_{U}$ and $\left.G\right|_{U}$ can be expressed as functions of each other.

\section{Toric integrability and generalizations of Gordon's theorem}

This is the main section in the paper. In $\S 5.1$ (Proposition 5.1.1) we show that if the system is torically integrable on a symplectic submanifold $N$ in the sense of Definition 4.2.2, then on $N$ one can introduce semilocal coordinates $(I, p, \varphi \bmod 2 \pi, q)$ normalizing the Hamiltonian of the system restricted to $N$. In $\S 5.2$ we construct and describe circular action functions $I=\left(I_{1}, \ldots, I_{k}\right)$ semilocally defined on any submanifold $N$ fibred into $k$-dimensional isotropic tori. In $\S 5.3$ we give the original statement of Gordon's theorem and show that it is a simple special case of Proposition 5.3.1, which is a geometric variant of Proposition 5.1.1.

In $\S 5.4$ we produce a counterexample to Gordon's theorem for the frequencies of a conditionally periodic motion on an integrability submanifold $N$ of some system. The existence of this counterexample is explained by the fact that the fibration of $N$ into the closed trajectories of the system is not integrable in the strong, Hamiltonian sense. Finally, in $\S 5.5$ we state the generalizations of Gordon's theorem for arbitrary submanifolds $N$ fibred into isotropic tori also of arbitrary dimension. We consider separately the case of action functions $I$, where the weakest condition, that of being pseudo-Hamiltonian is imposed on the fibration (see Theorem 5.5.1 A)), and the case of the frequencies $\omega$ of the conditionally periodic motion under the action of the flow of the system on the tori fibring $N$, where this fibration is required to be Hamiltonian (see Theorem 5.5.1 B)).

5.1. Normalizing coordinates $(I, p, \varphi \bmod 2 \pi, q)$ in a neighbourhood of a fibre of a toric fibration of a symplectic submanifold $N$. Proposition 5.1.1 stated below is the Hamiltonian analogue of Proposition 2.2.1 on the existence of the normalizing variables $(F, \varphi \bmod 2 \pi)$ in the case of a toric fibration of the phase space $N$ of a system of general form. In particular, it asserts that if a mixed set $\Phi=(Z, \mathcal{F})$ is Hamiltonian-torically integrable on a symplectic submanifold $N \subseteq M$, then, instead of the "coordinates" $(F, \varphi \bmod 2 \pi)$ that exist by Proposition 2.2.1, one can introduce much more convenient "coordinates" on $N$ semilocally, called generalized action-angle coordinates; see [5. These "coordinates" $(I, p, \varphi \bmod 2 \pi, q)$ are canonical with respect to the symplectic structure $\tilde{\omega}^{2}=\left.\omega^{2}\right|_{N}$ on $N$, and $I=I(Z)$. The Hamiltonian $\tilde{H}$ of the restriction to $N$ of a system Hamiltonian-torically $\Phi$-integrable on $N$ takes the simplest form $\tilde{H}=\tilde{H}(I)$ in these "coordinates". 
Proposition 5.1.1 is a simple consequence of the substantial main theorem in [5], which is stated and proved in $\S 1$ of that paper, and of its corollary stated at the end of that section. Note that the proofs of these two assertions in [5] are considerably more difficult than their non-Hamiltonian analogue, Proposition 2.2.1, which fact is related to the fact that the normalizing coordinates constructed in [5] are canonical. These assertions in [5] generalize the Liouville-Arnol'd theorem on toric integrability (see [1]) to the case of isotropic invariant tori $\Lambda$ of arbitrary dimension $k$, where $1 \leq k \leq n$, rather than tori that are necessarily Lagrangian, that is, of dimension $n$, where $2 n=\operatorname{dim} M$. Therefore the following assertion can also be regarded as a generalization of the original version of the Liouville-Arnol'd theorem in two directions: first, to the case of a symplectic submanifold $N$ of a nonintegrable system, and second, to the lower-dimensional case, that is, the case where $N$ is fibred into isotropic tori $\Lambda$ of any dimension $k=\operatorname{dim} \Lambda \leq(\operatorname{dim} N) / 2$.

Proposition 5.1.1. Let $\Phi=(Z, \mathcal{F})$ be some mixed set of functions Hamiltonian-torically integrable on a symplectic submanifold $N \subseteq M$; see Definition 4.2.1. As above, let $F=(\tilde{Z}, \mathcal{F})$ be the set of functions on $N$ associated with $\Phi$, where $\tilde{Z}=\left.Z\right|_{N}$. Recall that toric integrability means, in particular, that all the connected components $\Lambda$ of all the common level surfaces $F^{-1}(y) \subset N$ of the functions in $F$ are compact, as well as that $\operatorname{dim} N=2 k+l$, where $Z=\left(Z_{1}, \ldots, Z_{k}\right), \mathcal{F}=\left(\mathcal{F}_{1}, \ldots, \mathcal{F}_{l}\right), y \in \mathbf{R}^{k+l} ;$ see Definition 4.2.1. Then the following holds.

A) In some neighbourhood $U$ in $N$ of each such fixed component $\Lambda^{0}, \Lambda^{0} \subseteq F^{-1}(y) \subset N$, one can define generalized action-angle coordinates $(I, p, \varphi \bmod 2 \pi, q)$, that is, "coordinates" such that the restriction $\left.\omega^{2}\right|_{U}$ to $U \subseteq N$ of the symplectic form $\omega^{2}$ on $M$ can be written in terms of these variables in the form

$$
\left.\omega^{2}\right|_{U}=d\left(\sum_{i=1}^{k} I_{i} d \varphi_{i}+\sum_{j=1}^{l / 2} p_{j} d q_{j}\right) .
$$

Furthermore, the functions in the sets $(I, p, q)$ and $F=(\tilde{Z}, \mathcal{F})$ can be expressed as functions of each other in the neighbourhood $U \subseteq N$, as can the sets $I$ and $\tilde{Z}$ :

$$
(I, p, q)=\alpha(F), \quad F=\alpha^{-1}(I, p, q), \quad I=\beta(\tilde{Z}), \quad \tilde{Z}=\beta^{-1}(I) .
$$

Thus, the set $(I, p, \varphi \bmod 2 \pi, q)$ defines an isomorphism $U \rightarrow D^{k+l} \times \mathbf{T}^{k}$ of the domain $U \supset \Lambda^{0}$ onto the direct product of a domain $D^{k+l}$ of the linear space $\mathbf{R}^{k+l}$ and the $k$-dimensional torus $\mathbf{T}^{k}$. Furthermore, the fibrations of the domain $U \subseteq N$ defined by the sets $F$ and $(I, p, q)$ coincide: $F^{-1}(y) \cap U=\{(I, p, q)=\mathrm{const}\}$. One of these fibres $\Lambda=F^{-1}(y) \cap U$ coincides with $\Lambda^{0}$.

B) Suppose in addition that on $M$ there is a system with Hamiltonian function $H$ which is Hamiltonian- $\Phi$-integrable on a symplectic submanifold $N \subseteq M$; see Definition 4.2 .2 (for example, with $H=Z_{1}$ ). Then the vector field $J d H$ of this system is tangent to the submanifold $N$, that is, $J_{m} d H \in T_{m} M$ for all $m \in N$, and the restriction of this field to $N$ is a Hamiltonian system on the symplectic manifold $\left(N, \tilde{\omega}^{2}\right)$ with Hamiltonian function $\tilde{H}=\left.H\right|_{N}$, where $\tilde{\omega}^{2}:=\left.\omega^{2}\right|_{N}$. Furthermore, in the generalized action-angle variables $(I, p, \varphi \bmod 2 \pi, q)$ the function $\tilde{H}$ has the form $\tilde{H}=\tilde{H}(I)$, that is, it depends only on the action variables $I$. The tori $\{(I, p, q)=\mathrm{const}\}=\Lambda \subset U$ are invariant tori of this system, where the motion is conditionally periodic with frequencies $\omega_{1}, \ldots, \omega_{k}$ such that $\omega_{i}=\omega_{i}(I)=\frac{\partial \tilde{H}}{\partial I_{i}}(I), i=1, \ldots, k$.

Proof of Proposition 5.1.1. Lemma 3.1.1 reduces our situation of symplectic submanifold $N \subseteq M$ to that of a symplectic manifold $\left(N, \tilde{\omega}^{2}\right)$. This allows us to use the assertions in [5]. Proposition A) follows from the main theorem in [5] (see $\S 1$ there), while B) can be obtained from its corollary, stated at the end of $\S 1$ in [5], as well as from Lemma 4.2.1. 
A more precise and direct argument is as follows. Lemma 3.1.1 implies that $F=$ $(\tilde{Z}, \mathcal{F})$ is a Hamiltonian-torically integrable set of functions on the entire symplectic manifold $\left(N, \tilde{\omega}^{2}\right)$; see Definition 4.2.1. Applying the main theorem in [5] to $F$ we find that there exist semilocal canonical "coordinates" $(I, p, \varphi \bmod 2 \pi, q)$ on $\left(N, \tilde{\omega}^{2}\right)$, which are connected with the functions in the set $F=(\tilde{Z}, \mathcal{F})$ in the way described above. Using Lemma 3.1.1 again, with the sliding condition, we see that the Hamiltonian of the restricted system has the form $\tilde{H}=\left.H\right|_{N}$, and semilocally $\tilde{H}=\tilde{H}(I)$. This, with Hamilton's equations proves the assertion about the frequencies. Proposition 5.1.1 is proved.

5.2. Construction of circular action functions on a submanifold $N \subseteq M$ fibred into isotropic tori. Among the generalized action-angle variables $(I, p, \varphi \bmod 2 \pi, q)$ the action variables $I$ are the most important for the dynamics of a torically integrable system; they are also the easiest to construct. Given a set of functions integrable on the entire symplectic manifold $M$ the construction of the functions in the set $I=\left(I_{1}, \ldots, I_{k}\right)$ is given in [5. We describe it in a somewhat more general situation, when we have an arbitrary submanifold $N \subseteq M$, that is not necessarily symplectic, fibred into isotropic tori. Here we do not assume that there exist any vector fields tangent to the fibres of this fibration. Fundamentally this construction is little different from the construction given in $[5]$.

So, suppose we have a locally trivial fibration of an arbitrary submanifold $N \subseteq M$ whose fibres $\Lambda$ are $k$-dimensional isotropic tori, and no other conditions are imposed on this fibration. Let $U \subseteq N$ be an arbitrary open subset trivially fibred by the tori $\Lambda$. For example, any torus $\Lambda^{0} \subset N$ has a neighbourhood $U \subseteq N$ trivially fibred by the fibres $\Lambda$. We will define action functions $\left(I_{1}, \ldots, I_{k}\right)=I$ in $U$.

To do this, first we choose basis cycles $\gamma=\left(\gamma_{1}, \ldots, \gamma_{k}\right), \gamma_{i} \in \pi_{1}(\Lambda)$, compatibly on all the tori $\Lambda \subseteq U$, in the same way as in $\S 2.2$, where in a similar situation we defined the angular "functions" $\varphi \bmod 2 \pi$ in $U$ (see the proof of Proposition 2.2.1). Suppose that in a neighbourhood of $U$ in $M$ we have a 1-form $\alpha$ such that $d \alpha=\omega^{2}$. Then for the action functions we can take the integrals

$$
I_{i}(m)=\frac{1}{2 \pi} \oint_{\delta_{i}\left(\Lambda_{m}\right)} \alpha
$$

defined at any point $m \in U$; here $\delta_{i}\left(\Lambda_{m}\right)$ is any closed curve lying on the torus $\Lambda_{m} \ni m$ and representing the class $\gamma_{i}\left(\Lambda_{m}\right)$ of the fundamental group $\pi_{1}\left(\Lambda_{m}\right)$ of this torus. Instead of the 1-form $\alpha$ defined in a full domain of the manifold $M$ we can take a 1-form $\tilde{\alpha}$ defined only on $U \subseteq N$ itself and such that $d \tilde{\alpha}=\left.\omega^{2}\right|_{U}$.

The method of defining the action functions via a 1-form is the simplest, but there may be problems with the 1-form: for example, it may exist on $U$ but not be given explicitly. We give a more complicated method of defining the functions $I_{i}$ via integrals of the symplectic 2-form $\omega^{2}$ on the whole of $M$, which can be taken as given; this method always works. First we fix one of the tori $\Lambda \subseteq U$, which we denote by $\Lambda^{0}$, and choose any point $m \in U$. Consider any closed curves $\delta_{i}\left(\Lambda_{m}\right)$ and $\delta_{i}^{0}$ representing the 1-cycles $\gamma_{i}\left(\Lambda_{m}\right)$ and $\gamma_{i}^{0}$ on the tori $\Lambda_{m}$ and $\Lambda^{0}$, respectively. We stretch a 2-dimensional film $\Pi_{i}=\Pi_{i}\left(\Lambda_{m}\right)$ contained in $U$ over these curves. Then we have

$$
\delta_{i}\left(\Lambda_{m}\right) \subseteq \Lambda_{m}, m \in \Lambda_{m}, \quad \delta_{i}^{0} \subseteq \Lambda^{0}, \quad \partial \Pi_{i}=\delta_{i}\left(\Lambda_{m}\right)-\delta_{i}^{0},
$$

where $\partial \Pi_{i}$ is the boundary of the film $\Pi_{i}$. Since $\gamma_{i}\left(\Lambda_{m}\right)$ and $\gamma_{i}^{0}$ are, in fact, the same cycle but on different tori, such a film does exist. We set

$$
I_{i}(m)=\frac{1}{2 \pi} \iint_{\Pi_{i}\left(\Lambda_{m}\right)} \omega^{2}, \quad i=1, \ldots, k .
$$


Of course, instead of the form $\omega^{2}$ in these formulae one can take its restriction $\tilde{\omega}^{2}=\left.\omega^{2}\right|_{U}$ to $U \subseteq N$.

Proposition 5.2.1. The functions $I_{1}, \ldots, I_{k}$ are well defined in the following sense. The values of the integrals (12) do not depend either on the choice of the films $\Pi_{i} \subseteq$ $U$ connecting the closed curves $\delta_{i}\left(\Lambda_{m}\right) \subseteq \Lambda_{m}$ and $\delta_{i}^{0} \subseteq \Lambda^{0}$ or on the choice of these curves among the homotopy equivalent curves lying on the tori $\Lambda_{m}$ and $\Lambda^{0}$, respectively. Similarly, the values of the integrals (11) are independent of the choice of the closed curves $\delta_{i}\left(\Lambda_{m}\right) \subseteq \Lambda_{m}$. Furthermore, the functions $I_{1}, \ldots, I_{k}$ defined by (11) or (12) are constant on each torus $\Lambda_{m} \subseteq U$.

For fixed basis cycles $\gamma_{1}, \ldots, \gamma_{k}$ these functions are determined up to constants, which depend on the choice of the 1-form $\alpha$ such that $d \alpha=\omega^{2}$, or on the choice of the fixed torus $\Lambda^{0}$ over whose closed curves the 2 -film $\Pi_{i}$ is stretched from one side. The integrals of the 1-form differ from the integrals of the 2-form also only by constant summands.

If we consider two distinct sets of compatible basis cycles $\gamma_{1}, \ldots, \gamma_{k}$, then the corresponding sets $I=\left(I_{1}, \ldots, I_{k}\right)$ and $I^{\prime}=\left(I_{1}^{\prime}, \ldots, I_{k}^{\prime}\right)$ are expressed in terms of each other as follows: $I^{\prime}=A I+C$, where $C$ is a constant vector and $A$ is a unimodular matrix with integer coefficients, that is, $A=\left(a_{i j}, i, j=1, \ldots, k\right), \operatorname{det} A= \pm 1$, and $a_{i j} \in \mathbf{Z}$.

Consequently, the relations between different sets of circular action functions that held in the case of a Hamiltonian fibration of a symplectic manifold $M$ (see $\S 2$ in [5]) are still valid in this more general case when the fibration of an arbitrary submanifold $N \subseteq M$ into tori is simply isotropic.

The proposition obviously follows as $\omega^{2}: d \omega^{2}=0$ is closed and the leaves $\Lambda$ of the foliation are isotropic: $\left.\omega^{2}\right|_{\Lambda}=0$.

Definition 5.2.1. Suppose that an arbitrary submanifold $N \subseteq M$ of a symplectic manifold $M$ is locally trivially fibred into isotropic tori $\Lambda$. Let $U \subset N$ be an arbitrary open subset that is trivially fibred into these tori $\Lambda$. Then the functions $I_{1}, \ldots, I_{k}$ on $U$ defined by (11) or (12) are called the (circular) action functions corresponding to this fibration of $N \subseteq M$.

We emphasize that such functions can be constructed in a neighbourhood $U$ in $N$ of any torus $\Lambda \subseteq N$.

Remark 5.2.1. It is obvious how to generalise the construction of this set of circular action functions to the case of a locally trivial fibration of the submanifold $N \subseteq M$ with arbitrary isotropic fibres $\Lambda$, that is, not necessarily diffeomorphic to a torus; no problems arise and it is not even necessary that the fibres be compact. The only change required is that instead of the fundamental group $\pi_{1}(\Lambda)$ one must consider the group $H_{1}(\Lambda, \mathbf{Z})$ of 1-dimensional homologies and choose a basis $\gamma_{1}, \ldots, \gamma_{k}$ in this group, assuming that it exists. As in the case of tori, in the general case, when an initial fibre $\Lambda^{0}$ is fixed, there is a linear correspondence between the elements $\gamma$ of the homology group $H_{1}(\Lambda, \mathbf{Z})$ and the values (12) of the integral $I_{\gamma}$. Namely, if $\gamma=\sum_{i=1}^{k} a_{i} \gamma_{i}, a_{i} \in \mathbf{Z}$, then $I_{\gamma}=\sum_{i=1}^{k} a_{i} I_{i}$, where $I_{i}=I_{\gamma_{i}}, I_{\gamma}(m)=\frac{1}{2 \pi} \iint_{\Pi_{\gamma}} \omega^{2}$, and $\Pi_{\gamma}$ is a 2-film stretched over the cycles lying on the fibres $\Lambda_{m}$ and $\Lambda^{0}$ and corresponding to the element $\gamma \in H_{1}(\Lambda, \mathbf{Z})$. The same relation also holds for the values of the integral (11) for a fixed 1-form $\alpha$.

5.3. Generalization of Gordon's theorem to the case of a Hamiltonian fibration of a symplectic submanifold by compact fibres. Proposition 5.1.1 B) may be regarded as a generalization of the following well-known assertion.

Theorem 5.3.1 (Gordon's theorem; see 11, 12]). Suppose that all solutions of an autonomous system with Hamiltonian function $H$ are periodic and such that the trajectories 
of solutions of the system define a locally trivial fibration of the entire phase space $M$ with fibres diffeomorphic to a circle. Then semilocally, that is, in some neighbourhood in $M$ of each trajectory of the system, the period $T$ and the frequency $\omega=2 \pi / T$ of the solutions with trajectories contained in this neighbourhood are functions of the Hamiltonian $H$ only: $T=T(H)$ and $\omega=\omega(H)$.

The circular action function $I$, defined above via the integral of the symplectic structure $\omega^{2}$, also depends only on $H$ and is unique in this situation, that is, semilocally $I=I(H)$. Moreover, $T(H)=2 \pi \frac{d I}{d H}(H)$.

In order to make the connection between Gordon's theorem and Proposition 5.1.1 B) clearer we state the following proposition, which can be viewed as a reformulation of Proposition 5.1.1 B) in geometric terms of fibrations.

Proposition 5.3.1 (Generalization of Gordon's theorem to the case of fibrations of a symplectic submanifold by invariant isotropic tori of arbitrary dimension). Suppose that a system with Hamiltonian function $H$ is defined on a symplectic manifold $M$, and there is a symplectic submanifold $N \subseteq M$ and a set $Z=\left(Z_{1}, \ldots, Z_{k}\right)$ of functions on the whole of $M$ such that $H, Z$, and $N$ have the following properties.

a) At each point $m \in N$ the vector of the field $J d H$ is a linear combination of the vectors of the fields $J d Z_{1}, \ldots, J d Z_{k}: \quad J_{m} d H=\sum_{i=1}^{k} \lambda_{i} J_{m} d Z_{i}, \lambda_{i}=\lambda_{i}(m)$, $m \in N$.

b) $\left.\left\{Z_{i}, Z_{j}\right\}\right|_{N}=0$.

c) $N$ is invariant under the local phase flows $\left\{g_{Z_{i}}^{t_{i}}\right\}$ corresponding to the Hamiltonian fields $J d Z_{i}, i=1, \ldots, k$. Furthermore, the orbits $\Lambda_{m}, m \in \Lambda_{m}$, of the joint action of these flows lying on $N$ are compact, $k$-dimensional, and form a locally trivial fibration of the submanifold $N$.

Then the following two assertions hold.

A) For all $m \in M$ the orbits $\Lambda=\Lambda_{m} \subset N$ are invariant $k$-dimensional isotropic tori of the system, on which the motion is conditionally periodic. The functions in the set $Z$ are constant on each such orbit $\Lambda$. Furthermore, semilocally, that is in some neighbourhood $U \subseteq N$ in $N$ of each such orbit, the frequencies $\left(\omega_{1}, \ldots, \omega_{k}\right)=\omega$ of this motion, as well as the restriction $\left.H\right|_{N}$ of the Hamiltonian function $H$ to $N$, depend only on the values of the functions $Z_{1}, \ldots, Z_{k}$ on any orbit $\Lambda \subset U$, so that

$$
\omega=\omega(\tilde{Z}), \quad \tilde{H}=\tilde{H}(\tilde{Z}), \quad \text { where } \tilde{Z}=\left(\tilde{Z}_{1}, \ldots, \tilde{Z}_{k}\right), \tilde{Z}_{i}=\left.Z_{i}\right|_{U}, \quad \tilde{H}=\left.H\right|_{U} .
$$

B) The same is also true for the circular action functions $\left(I_{1}, \ldots, I_{k}\right)=I$ defined on $U$ (see Definition 5.2.1), that is, semilocally $I=I(\tilde{Z})$. The converse dependence also holds, that is, semilocally $\tilde{Z}=\tilde{Z}(I)$. Furthermore, $\omega(I)=\frac{\partial \tilde{H}}{\partial I}(I)$ on the tori $\Lambda \subset U \subseteq N$.

Gordon's theorem is a special case of this assertion and is obtained from it by setting $k=1$ and $N=M$ and taking $Z_{1}=H$ for the function $Z_{1}$.

Proposition 5.3.1 can be fairly easily derived from Proposition 5.1.1. The main difficulty consists in proving the existence of a regular set $F=(\tilde{Z}, \mathcal{F})$ of functions on $U$ specifying the fibres $\Lambda \subset U$ by their common level surfaces. (Roughly speaking the same difficulty must be overcome in the proof of the converse assertion in Proposition 4.3.1; see also Remark 4.3.1.) The regularity of the set $Z$ on $N$ follows from the fact the fibres $\Lambda$ are $k$-dimensional. This with Lemma 3.5.1 implies that $\left.Z\right|_{N}$ is regular and therefore so is $\tilde{Z}=\left.Z\right|_{U}$. The involutivity of $Z$ on $N$ implies that $\tilde{Z}$ is constant on each fibre $\Lambda$ contained in $U$. The existence and regularity of a whole set $F=(\tilde{Z}, \mathcal{F})$ such that the 
fibres $\Lambda \subset U$ coincide with the inverse images $F^{-1}(y)$ of points $y \in \mathrm{R}^{r}$ follows from this and the fact that the fibres $\Lambda$ that form a locally trivial fibration of $N$ are compact and connected; here $r=\operatorname{dim} N-k$. This is what makes it possible to apply Proposition 5.1.1.

The fact that the circular action functions $\left(I_{1}, \ldots, I_{k}\right)=I$ coincide with the action variables that form a part of the generalized action-angle variables follows from Proposition 5.2.1 and the expression of the form $\left.\omega^{2}\right|_{U}$ in these variables; see Proposition 5.1.1 A). Furthermore, Proposition 5.3.1 is an obvious corollary of Theorem 5.5.1 B) stated below; see Remark 5.5.7.

5.4. Counterexample to Gordon's theorem in the case of a nonsymplectic fibred submanifold $N \subset M$. Using Proposition 3.3.3 one can easily show that the conditions imposed on the fibration of the symplectic submanifold $N \subseteq M$ in the preceding proposition were the conditions of a pseudo-Hamiltonian toric fibration (see Definition 3.2.3) whose fibres are invariant under the phase flow of the system. The question arises as to whether Gordon's theorem remains valid in the case of a general submanifold $N \subseteq M$, that is, one that is not necessarily symplectic, if we impose the same conditions of a pseudo-Hamiltonian fibration of the submanifold $N$ with invariant fibres diffeomorphic to the torus, that is, if we require that all the orbits $\Lambda$ of the joint action of the flows of the vector fields $J d Z_{1}, \ldots, J d Z_{k}$ intersecting $N$ are contained in $N$ and are $k$-dimensional invariant isotropic tori forming a locally trivial fibration of $N$ ? The following simple example shows that this question has a negative answer even when stronger conditions are imposed on the fibration.

Counterexample. Consider the integrable system with Hamiltonian $H=J_{1} J_{2}$ defined on the 4-dimensional symplectic manifold $M^{4}=A_{J}^{2} \times \mathbf{T}_{\psi}^{2}$, where $A_{J}^{2}=\mathbf{R}_{J}^{2} \backslash\{0\}$ is the two-dimensional plane with zero removed, and $(J, \psi \bmod 2 \pi)$ are the canonical actionangle "coordinates". For $N \subset M$ we take the 3-dimensional surface

$$
N=N^{3}=\left\{(J, \psi \bmod 2 \pi) \in M^{4} \mid J_{2}=0, J_{1}>0\right\} .
$$

Then the frequencies $\Omega_{1}, \Omega_{2}$ of the conditionally periodic motion on the invariant 2dimensional tori $\{J=$ const $\}$ contained in $N$ have the form

$$
\Omega_{1}=\frac{\partial H}{\partial J_{1}}(J)=J_{2}=0, \quad \Omega_{2}=\frac{\partial H}{\partial J_{2}}(J)=J_{1} \neq 0 .
$$

Thus, the connected submanifold $N^{3} \subset M^{4}$ is trivially fibred into the closed trajectories of the system and lies on an isoenergetic surface, namely, $N^{3} \subset H^{-1}(0)$. But the frequency $\omega$ of the periodic solutions whose trajectories form a fibration of $N$ is not constant: $\omega=\Omega_{2}=J_{1} \neq$ const on $N$. This contradicts the hypothetical generalization of Gordon's theorem according to which if $N \subset M$ has a fibration into closed trajectories $\gamma$, then $\omega=\omega(H)$ in a neighbourhood of each such trajectory. Since $N=N^{3}$ is connected, this would mean that $\omega=$ const; compare with Theorem 5.3.1 or with Proposition 5.3.1 with $Z_{1}=H$.

Remark 5.4.1. The fibration of $N$ into the trajectories $\gamma=\Lambda$ of the system with Hamiltonian $Z_{1}=H$ is not merely pseudo-Hamiltonian but also weakly Hamiltonian and toric. However, even this is not enough for Gordon's theorem to hold in the case of nonsymplectic $N$. It is necessary that it be Hamiltonian; this follows if it is pseudo-Hamiltonian when $N$ is symplectic; see Corollary 3.3.1.

Remark 5.4.2. Complete integrability of the system, that is, integrability over the entire phase space $M^{4}$, is completely unnecessary in the example considered above. This is shown by the following argument. It is easy to "corrupt" the Hamiltonian function $H=J_{1} J_{2}$ so that the vector field of the system and the function $H$ remain the same on 
$N$ and therefore the conclusion of Gordon's theorem remains invalid. At the same time, the arbitrariness in the choice of such a deformation of the function $H$ is so great that among the resulting systems there certainly exist systems that are not integrable on the whole of $M$. It would appear that this must be possible even in the analytic situation.

Remark 5.4.3. In this example, unlike the frequency $\omega$, the circular action function $I_{1}$ corresponding to the fibration of $N$ into the closed trajectories of the system is constant:

$$
I_{1}(m)=\frac{1}{2 \pi} \oint_{\gamma_{m}} J d \psi=\frac{1}{2 \pi} \oint_{\gamma_{m}} J_{2} d \psi_{2}=J_{2}=0 .
$$

Here $\gamma_{m} \ni m$ is the trajectory of the system passing through a point $m=(J, \psi \bmod 2 \pi)$, and the middle equality holds because $\psi_{1}=$ const on the curve $\gamma_{m}$. The fact that the action is constant on such $N$ fully agrees with Theorem 5.5.1 A) stated below.

5.5. Generalizations of Gordon's theorem to the general case of a submanifold that is not necessarily symplectic, fibred into tori. Below we state the two main theorems of the paper. In particular these show the following. The fact that the circular action functions $I=I(\tilde{Z})$ only depend on the functions in the original set $Z$, which is in involution on $N$ (or more precisely on the set $\tilde{Z}=\left.Z\right|_{U}$ ), is also locally true for an arbitrary submanifold $N \subseteq M$ that is not necessarily symplectic. The requirements on the fibration $\Xi_{N}(Z)$ are minimal: it must be pseudo-Hamiltonian and toric; see Theorem 5.5.1 A). Here $U \subseteq N$ is some neighbourhood in $N$ of an arbitrary torus-fibre of this fibration.

But to prove the converse dependence $\tilde{Z}=\tilde{Z}(I)$ we need to place a much stronger condition on $N$ and $Z$. Namely, the dependence of the form $\tilde{Z}=\tilde{Z}(I)$ is guaranteed by a Hamiltonian fibration of the submanifold $N$ into tori; see Theorem 5.5.1 B) where we take $H=Z_{1}$. In other words, compared with Theorem 5.5.1 A) we must assume in addition that $\operatorname{rank}\left(\left.Z\right|_{N}\right)_{*}=k$ instead of $\operatorname{rank} Z_{*}=k$ on $N$. If there is an arbitrary system whose vector field is tangent to the fibres of the fibration $\Xi_{N}(Z)$, then these conditions on $\Xi_{N}(Z)$ are sufficient for the dependence $\omega(I)=\frac{\partial \tilde{H}}{\partial I}(I)$. Here $H$ is the Hamiltonian of the system, $\tilde{H}=\left.H\right|_{U}$, and $\omega$ is the set of frequencies of the conditionally periodic motion of the system on the invariant fibres-tori of this fibration of $N$.

Theorem 5.5.1. A) (Generalization of Gordon's theorem for circular action functions $I$ on an arbitrary submanifold $N \subseteq M)$ Suppose there is a set of functions $Z=\left(Z_{1}, \ldots, Z_{k}\right)$ on a symplectic manifold $\left(M, \omega^{2}\right)$ and a submanifold $N \subseteq M$ with the following property. Let $\mathcal{J} d Z$ denote the field of the linear spans of the vectors of the $k$ Hamiltonian fields $J d Z_{1}, \ldots, J d Z_{k}$. Suppose that the field of planes $\mathcal{J} d Z$ is tangent to $N$ and is a $k$-dimensional Frobenius integrable distribution on $N$ whose integral surfaces are isotropic $k$ dimensional tori that form a locally trivial fibration of $N$.

Let $\Lambda^{0} \subseteq N$ be an arbitrary fixed leaf of this foliation and let $I=\left(I_{1}, \ldots, I_{k}\right)$ be some set of circular action functions defined in some neighbourhood $U$ of the torus $\Lambda^{0}$ in $N$; see Definition 5.2.1. Let $\sigma$ be an arbitrary connected smooth submanifold of the manifold $N$ contained in the intersection $U \cap Z^{-1}(z)$ of this neighbourhood $U \subseteq N$ with some common level surface $Z^{-1}(z)$ of the functions in the set $Z$ : $\sigma \subseteq U \cap Z^{-1}(z)$. Suppose that this surface $\sigma$ has the following completeness property: for each point $m \in \sigma$ the entire fibre $\Lambda_{m} \subseteq N$ of our fibration passing through this point lies in $\sigma$, so that we have

$$
m \in \Lambda_{m} \subseteq \sigma \subseteq U \cap Z^{-1}(z) \subseteq N
$$

Then, under all these conditions, the action functions are constant on this submanifold: $\left.I\right|_{\sigma}=C, C \in \mathbf{R}^{k}$. 
Remark 5.5.1. We look at the subset $\sigma$ instead of the entire intersection $U \cap Z^{-1}(z)$ because the intersection $N \cap Z^{-1}(z)$ may be quite a nonregular subset in $N$ (cf. Remark 3.2.3), and in the proof of this theorem we have to consider smooth films contained in $\sigma$.

We also note that since the fibration under consideration is isotropic, the completeness property $m \in \sigma \Rightarrow \Lambda_{m} \subseteq \sigma$ is not too restrictive. Indeed, we can assume that the intersection $U \cap Z^{-1}(z)$ containing $\sigma$ has this property itself. Indeed, by definition the fibres $\Lambda_{m}$ are entirely contained in $N$, and it is clear that the neighbourhood $U \subseteq N$ of the torus $\Lambda^{0}$ can be chosen so that $U$ has the same property. The isotropy of the fibres $\Lambda$ and Proposition 3.3.3 imply that the functions in the set $Z$ are constant on each of them, that is, these fibres are entirely contained in the common level surfaces $Z^{-1}(z)$. Thus, the subsets $N \cap Z^{-1}(z)$ and $U \cap Z^{-1}(z)$ have the completeness property.

In particular, consider the case where the action variables $I$ are defined globally on the whole of $N$, that is, $U=N$. Then any connected component of the intersection $N \cap Z^{-1}(z)$ that is a smooth submanifold of the manifold $N$, or a connected smooth stratum of such a component, satisfies all the conditions imposed on $\sigma$ in the theorem.

Remark 5.5.2. The proof of Theorem 5.5.1 A) given in $\S 6.4$ shows that one can weaken the smoothness condition on the subset $\sigma \subseteq U \cap Z^{-1}(z)$, replacing it by the weaker condition that any two points of this subset can be connected by a path contained in $\sigma$ that is piecewise smooth in $N$.

We also note that if a submanifold $\sigma \subseteq N$ of the surface $N$ is smooth in $N$, then it is smooth in the whole of $M \supseteq N$.

Remark 5.5.3. The main condition imposed on $N$ and $Z$ in Theorem A) is equivalent to requiring that the set $Z$ define a pseudo-Hamiltonian fibration of $N \subseteq M$ into $k$-dimensional tori. This follows immediately from Definition 3.2.3 of such fibrations, as they are locally trivial; see Remark 3.2.0.

Theorem 5.5.1. B) (Generalization of Gordon's theorem for the frequencies of a conditionally periodic motion on an arbitrary integrability submanifold $N \subseteq M$ ) Suppose that on a symplectic manifold $\left(M, \omega^{2}\right)$ a system with Hamiltonian $H$ is defined that is Hamiltonian-torically $Z$-integrable on some submanifold $N \subseteq M$ in the sense of Definition 3.2.4, where $Z=\left(Z_{1}, \ldots, Z_{k}\right)$, or Hamiltonian-torically $\Phi$-integrable on $N \subseteq M$ in the sense of Definition 4.2.2, where $\Phi=(Z, \mathcal{F})$ and $Z$ is again a set of $k$ functions on $M$. Let $\Lambda$ denote the fibres of the corresponding $k$-dimensional fibration of the submanifold $N$; in the first case this is the fibration $\Xi_{N}(Z)$ into the integral surfaces of the distribution defined by the vector fields $J d Z_{1}, \ldots, J d Z_{k}$, and in the second case, the fibration $\Xi_{N F}$ into the connected components of the inverse images of points under the map $F: N \rightarrow \mathbf{R}^{k+l}$, where $F=\left(\left.Z\right|_{N}, \mathcal{F}\right)$.

Then in either case the fibres $\Lambda$ of this Hamiltonian fibration of $N$ are invariant $k$ dimensional isotropic tori of the system forming a locally trivial fibration of $N$, and the motion on these tori is conditionally periodic. Each function in $Z$ is constant on any fibre $\Lambda$ of this fibration. Furthermore, each fixed torus-fibre $\Lambda^{0} \subset N$ has a neighbourhood $U$ in $N, \Lambda^{0} \subset U \subseteq N \subseteq M$, having the following properties.

The frequencies $\omega=\left(\omega_{1}, \ldots, \omega_{k}\right)$ of such a motion on the tori $\Lambda$ contained in $U$, and the restriction $\tilde{H}:=\left.H\right|_{U}$, depend only on the set $\tilde{Z}:=\left.Z\right|_{U}$ of functions in the set $Z$ restricted to $U$, that is, $\omega=\omega(\tilde{Z})$ and $\tilde{H}=\tilde{H}(\tilde{Z})$. Furthermore, a set of circular action functions $I$ can be defined on $U$ (see Definition 5.2.1), and this set and the set $\tilde{Z}$ can be expressed as functions of each other: $I=I(\tilde{Z})$ and $\tilde{Z}=\tilde{Z}(I)$. In addition, the 
frequencies $\omega$ on $U \subseteq N$ are connected with $\tilde{H}$ and $I$ by the relations $\omega_{i}(I)=\frac{\partial \tilde{H}}{\partial I_{i}}(I)$, $i=1, \ldots, k$.

Remark 5.5.4. Definitions 3.2.4 and 4.2.2 concerning Hamiltonian toric $Z$-integrability and $\Phi$-integrability on the submanifold $N \subseteq M$ are, in fact, equivalent; see Remark 4.3.1 and Proposition 4.3.1 B), which we have not yet proved. In particular, in some neighbourhood $U$ in $N$ of any fibre $\Lambda^{0}$ of the toric fibration $\Xi_{N}(Z)$ defined by the set of vector fields $J d Z$ (see Definitions 3.2.4 and 3.2.2) there exists a set of functions $\mathcal{F}$ defined on $U \subseteq N$ and such that the set $\Phi=(Z, \mathcal{F})$ is Hamiltonian-integrable on $U$ (see Definitions 4.2.2 and 4.2.1). Here the fibres $\Lambda \subset U$ of the fibration $\Xi_{N}(Z)$ coincide with the fibres of the fibration $\Xi_{N F}$ into the connected components of the inverse images of points under the map $F: N \rightarrow \mathbf{R}^{k+l}$, where $F$ is the set associated with $\Phi$, that is, $F=\left(\left.Z\right|_{U}, \mathcal{F}\right)$.

Remark 5.5.5. Recall that the existence of a Hamiltonian fibration of $N$ implies that $\operatorname{dim} N \geq 2 k$. Recall also that according to Corollary 3.4.1 and Theorem 3.4.1B) the verification of the conditions of Theorem B) can be simplified by omitting conditions c) and $\mathrm{f}$ ) in Definition 3.2.4 of Hamiltonian integrability, which together mean that the vector fields $J d H, J d Z_{1}, \ldots, J d Z_{k}$ commute pairwise on $N$.

We now compare the conditions in Theorems 5.5.1 A) and B), taking into account that the conditions in Theorem B) have been weakened. We see that in Theorem B), in addition to the conditions of Theorem A), the condition that $\left.Z\right|_{N}$ be regular is imposed on $Z$. In Theorem B) it is also assumed that there exists a system on $M$ on which only one condition is imposed - the sliding condition, that is, it is assumed that $d H=\sum_{i=1}^{k} \lambda_{i} d Z_{i}$ at every point $m \in N$, where $\lambda_{i}=\lambda_{i}(m)$ and $H$ is the Hamiltonian of the system. Compared with Theorem A), no other conditions are imposed. Thus, in this context the condition that $\left.Z\right|_{N}$ be noncritical can be regarded as the most important condition among those imposed on $Z$ and $N \subseteq M$ in Theorem B).

The fact that this condition is essential is shown by the counterexample to Gordon's theorem given above, which does not contradict Theorem B) precisely because the regularity condition on the set $\left.Z\right|_{N}$, which consists of the single function $\left.Z_{1}\right|_{N}$, does not hold there. Indeed, in that example we have $\left.Z_{1}\right|_{N}=0$, where $Z_{1}=H$. In other words, the fibration of $N^{3}$ into the closed trajectories $\gamma$ of the system with Hamiltonian $H$ "cannot be continued outside the surface $\{H=0\}$ ".

The existence of this counterexample can also be explained by the fact that for each trajectory $\gamma \subset N$ there is no symplectic 2-dimensional submanifold $S \subseteq N$ containing $\gamma$ and composed entirely of closed trajectories of the system. This means that the condition that the fibration $\Xi_{N}(Z)$ of $N$ be quasi-Lagrangian is not satisfied. The existence of all these slightly different explanations corresponds to the assertion of Proposition 3.4.1 A) that the condition that $\left.Z\right|_{N}$ is regular and the condition that the fibration of $N$ defined by the set $Z$ is quasi-Lagrangian are equivalent.

Remark 5.5.6. We emphasize that in Theorems A) and B) it is not required that the rank of the 2-form $\tilde{\omega}^{2}$ on $N$ be constant, where $\tilde{\omega}^{2}=\left.\omega^{2}\right|_{N}$ is the restriction to $N$ of the symplectic structure $\omega^{2}$ defined on $M$.

Remark 5.5.7. Proposition 5.3.1, which generalizes Gordon's theorem to the case of a symplectic integrability submanifold $N$ follows easily from Theorem 5.5.1 B). Indeed, since $N \subseteq M$ is symplectic, it follows from Corollary 3.3.1 that the system is Hamiltoniantorically $Z$-integrable on $N$. This fact and the assertions of Theorem 5.5.1 B) imply all the assertions of Proposition 5.3.1. 


\section{Proofs of the Assertions in $\S \S 3-5$}

In this section we shall prove all the assertions stated above which were not proved straight away, namely, Propositions 3.4.1 A) and B), 4.3.1 A) and B), 4.3.2, and Theorems 5.5.1 A) and $\mathrm{B}$ ).

6.1. Auxiliary assertions. For the proofs we will need the following facts from linear symplectic geometry.

Lemma 6.1.1. Let $Q \subseteq \mathbf{L}^{2 n}$ be an arbitrary isotropic subspace of a linear symplectic space $\left(\mathbf{L}^{2 n}, \Omega^{2}\right)$, where $\Omega^{2}$ is a linear symplectic structure on a linear $2 n$-dimensional space $\mathbf{L}^{2 n}$, that is, a bilinear nondegenerate skew-symmetric 2 -form. Let $P=Q^{\perp}$ be the skew-orthogonal complement of $Q$; see [1]. Then the following holds.

A) $P \supseteq Q$.

B) Suppose that in addition we have an arbitrary subspace $B$ of $\mathbf{L}^{2 n}$ containing $Q$, that is, $Q \subseteq B \subseteq \mathbf{L}^{2 n}$. Let $K$ denote the kernel of the restriction of the form $\Omega^{2}$ to $B$, that is, the subspace $K=B \cap B^{\perp}$. Then $B$ and $P$ are transversal to each other, that is, the vectors in $B$ and $P$ generate the entire space $\mathbf{L}^{2 n}$ if and only if the linear subspaces $K$ and $Q$ contained in $B$ intersect only in zero, $0 \in \mathbf{L}^{2 n}$ :

$$
\operatorname{span}(B, P)=\mathbf{L}^{2 n} \Longleftrightarrow K \cap Q=\{0\} .
$$

Here $\operatorname{span}(B, P)$ denotes the linear span of the subspaces $B$ and $P$.

C) Suppose that $R \subset \mathbf{L}^{2 n}$ is an arbitrary $k$-dimensional subspace transversal to the $(2 n-k)$-dimensional subspace $P$ where $k=\operatorname{dim} Q$, that is, $R \oplus P=\mathbf{L}^{2 n}$. Then the direct sum $Q \oplus R$ of the subspaces $Q$ and $R$ is a symplectic $2 k$-dimensional subspace of the space $\mathbf{L}^{2 n}$, that is, the bilinear form $\left.\Omega^{2}\right|_{Q \oplus R}$ is nondegenerate.

Proof of Lemma 6.1.1. Since $Q$ is isotropic, $\left.\Omega^{2}\right|_{Q}=0$; consequently, all the vectors in $Q$ are skew-orthogonal to $Q$, that is, $Q \subseteq Q^{\perp}=P$, which proves assertion A) of the lemma.

Let $A \subseteq \mathbf{L}^{2 n}$ and $B \subseteq \mathbf{L}^{2 n}$ be any two subspaces. Since the 2 -form $\Omega^{2}$ is nondegenerate, it follows that if $A \subset B$, then $A^{\perp} \supset B^{\perp}$. Using this fact it is easy to verify that $(A \cap B)^{\perp}=\operatorname{span}\left(A^{\perp}, B^{\perp}\right)$. Indeed,

$$
A \cap B \subseteq A \Longrightarrow(A \cap B)^{\perp} \supseteq A^{\perp} \Longrightarrow(A \cap B)^{\perp} \supseteq \operatorname{span}\left(A^{\perp}, B^{\perp}\right) .
$$

On the other hand,

$$
\begin{aligned}
A^{\perp} \subseteq \operatorname{span}\left(A^{\perp}, B^{\perp}\right) & \Longrightarrow A \supseteq\left(\operatorname{span}\left(A^{\perp}, B^{\perp}\right)\right)^{\perp} \Longrightarrow \\
A \cap B \supseteq\left(\operatorname{span}\left(A^{\perp}, B^{\perp}\right)\right)^{\perp} & \Longrightarrow(A \cap B)^{\perp} \subseteq \operatorname{span}\left(A^{\perp}, B^{\perp}\right) .
\end{aligned}
$$

From this, it is easy to deduce that $(A \cap B \cap C)^{\perp}=\operatorname{span}\left(A^{\perp}, B^{\perp}, C^{\perp}\right)$. (In fact, the number of subspaces in this formula can be arbitrary and the formula remains valid, but two and three suffices for us.) Using these facts we prove assertion B). The embedding $B \supseteq Q$ implies that $B^{\perp} \subseteq Q^{\perp}=P$. On the other hand, as $B \cap B^{\perp} \cap Q=\{0\}$, thus $\operatorname{span}\left(B^{\perp}, B, P\right)=\mathbf{L}^{2 n}$. From these two assertions we obtain $\operatorname{span}(B, P)=\mathbf{L}^{2 n}$, that is, the subspaces $B$ and $P$ are transversal. Conversely, if $\operatorname{span}(B, P)=\mathbf{L}^{2 n}$, then $B^{\perp} \cap P^{\perp}=\{0\}$ and therefore $K \cap Q=B \cap B^{\perp} \cap Q=\{0\}$. Assertion B) is proved.

To prove $\mathrm{C}$ ) we observe that $Q$ and $R$ intersect in zero, $0 \in \mathbf{L}^{2 n}$, since $Q \subseteq P$ and $R$ intersects $P$ in zero. Therefore we can indeed speak about the direct $\operatorname{sum} Q \oplus R$. We prove by contradiction that the $2 k$-dimensional subspace $Q \oplus R$ is symplectic. Indeed, suppose that there exists a nonzero vector $\xi \in Q \oplus R$ that is skew-orthogonal to this entire subspace, that is, $\xi \in(Q \oplus R) \cap(Q \oplus R)^{\perp}$. This means that $\xi \in(Q \oplus R)^{\perp} \subset Q^{\perp}=P$. Consequently, $\xi \in(Q \oplus R) \cap P$. But by the hypothesis of Lemma $\mathrm{C})$ the subspace $R$ intersects $P$ in zero; hence $Q \oplus R$ intersects $P$ in $Q$ and therefore $\xi \in Q$. 
Since $R \oplus P=\mathbf{L}^{2 n}$, the subspace $B:=Q \oplus R$ is also transversal to the subspace $P$, that is, $\operatorname{span}(B, P)=\mathbf{L}^{2 n}$. This fact and the formula connecting the linear span and the intersection of subspaces imply that $B^{\perp} \cap Q=\{0\}$. But $\xi \in B \cap B^{\perp} \subseteq B^{\perp}$ and therefore $\xi \in B^{\perp} \cap Q$. These two facts imply $\xi=0$. The contradiction thus obtained proves assertion $\mathrm{C}$ ). The proof of Lemma 6.1.1 is complete.

Let $F=\left(F_{1}, \ldots, F_{r}\right)$ be an arbitrary regular set of functions defined on a $2 n$-dimensional symplectic manifold $\left(M, \omega^{2}\right), M=M^{2 n}$. It is clear that in this case the common level surfaces $F^{-1}(b)$ of the functions in $F$ are smooth $(2 n-r)$-dimensional surfaces at each point $m \in M$. We denote by $\mathcal{J}_{m} d F$ the subspace of the tangent space $T_{m} M$ to $M$ at a point $m \in M$ spanned by the vectors of the Hamiltonian fields $J d F_{1}, \ldots, J d F_{r}$ at this point. We denote by $\mathcal{J} d F$ the corresponding distribution of $k$-dimensional planes, that is, of the planes $\mathcal{J}_{m} d F$. (These should not be mixed up with $J d F$, by which we meant the set of vector fields $J d F_{1}, \ldots, J d F_{r}$.)

Lemma 6.1.2. A) At every point $m \in M$ the tangent space $T_{m}\left(F^{-1}(b)\right)$ to the common level surface $F^{-1}(b) \ni m$ and the subspace $\mathcal{J}_{m} d F$ are the skew-orthogonal complements of each other in the linear symplectic space $\left(T_{m} M, \Omega_{m}^{2}\right)$, where $\Omega_{m}^{2}=\left.\omega^{2}\right|_{T_{m} M}$ and $T_{m} M$ is the tangent space to $M$ at the point $m$. In other words, $\left(\mathcal{J}_{m} d F\right)^{\perp}=T_{m}\left(F^{-1}(b)\right)$.

B) If the functions in the set $F$ are pairwise in involution at a point $m$, that is, $\left\{F_{i}, F_{j}\right\}=0, i, j=1, \ldots, r$, at this point, then the $r$-dimensional subspace $\mathcal{J}_{m} d F$ is isotropic: $\left.\Omega_{m}^{2}\right|_{\mathcal{J}_{m} d F}=0$. Furthermore, $\mathcal{J}_{m} d F \subseteq T_{m} F^{-1}(b)$.

Proof. The proof of the lemma is obvious. Indeed, let $\xi \in T_{m}\left(F^{-1}(b)\right)$; then we have $\omega^{2}\left(\xi, J d F_{i}\right)=d F_{i}(\xi)=0, i=1, \ldots, r$; see the beginning of $\S 3.1$. Consequently, any vector in $T_{m}\left(F^{-1}(b)\right)$ is skew-orthogonal to the vectors $J d F_{1}, \ldots, J d F_{r}$ and therefore to their linear span $\mathcal{J}_{m} d F$, that is, the subspaces $T_{m}\left(F^{-1}(b)\right)$ and $\mathcal{J}_{m} d F$ are skeworthogonal. These subspaces have dimension $2 n-r$ and $r$ respectively, that is, they are complementary in $T_{m} M$; they are therefore skew-orthogonal complements, which proves assertion A). Assertion B) on isotropy is an obvious consequence of involutivity and (4), and the embedding $\mathcal{J}_{m} d F \subseteq T_{m} F^{-1}(b)$ is an obvious consequence of Lemma 6.1.1 A) and the fact that these two subspaces are skew-orthogonal complements.

6.2. Proof of Propositions 3.4.1 A) and B). We now prove Proposition 3.4.1 A), that is, that conditions a), b), c), and d) of this proposition are equivalent. The equivalence of Definition 3.4.1 a) and c) (nondegenerate isotropy and regularity of the set of functions $\tilde{Z}$ respectively) is an easy consequence of assertions A) and B) of Lemma 6.1.1 and both assertions of Lemma 6.1.2.

Indeed, in Lemma 6.1.1 we take $Q=\mathcal{J}_{m} d Z, P=T_{m}\left(Z^{-1}(z)\right)$, and $B=T_{m} N$, where $m \in N$ is any point and $z=Z(m)$. Lemma 6.1.2 A) implies that $P=Q^{-}$. From Lemma $6.1 .2 \mathrm{~B}$ ) and the involutivity of the set $Z$ on $N$ we obtain the isotropy of $Q$ and therefore the isotropy of our pseudo-Hamiltonian foliation $\Xi_{N}(Z)$ defined by the distribution $\mathcal{J} d Z$. By the hypothesis of Proposition 3.4.1 A) the set $Z$ is regular on $N$; hence condition c) that the set $\tilde{Z}=\left.Z\right|_{N}$ is regular on $N$ is equivalent to the fact that the subspaces $T_{m} N$ and $T_{m}\left(Z^{-1}(z)\right)$ are transversal; see Remark 3.2.3. In other words, condition c) is equivalent to the equality $\operatorname{span}(B, P)=\mathbf{L}^{2 n}$, where, as above, $\operatorname{span}(B, P)$ is the linear span of the subspaces $B$ and $P$. Furthermore, Lemma 6.1.1B) implies that $\operatorname{span}(B, P)=\mathbf{L}^{2 n} \Longleftrightarrow B \cap B^{\perp} \cap Q=\{0\}$. Together with the preceding equivalence this proves that a) $\Longleftrightarrow \mathrm{c}$ ).

The most cumbersome part is the proof of the fact that $c) \Longrightarrow b$ ), which we present below; we prove that the regularity of the set $\tilde{Z}$ implies that the foliation $\Xi_{N}(Z)$ is quasiLagrangian. We fix any point $m \in N$ and construct some $k$-dimensional surface $\Gamma \subset N$ 
through it such that the set $\left.Z_{1}\right|_{\Gamma}, \ldots,\left.Z_{k}\right|_{\Gamma}$ of the restrictions to $\Gamma$ of the functions in $Z$ is regular on $\Gamma$. In other words, we require that the set of these restrictions be a set of local coordinates on $\Gamma$. The condition that the set $\tilde{Z}=\left(\tilde{Z}_{1}, \ldots, \tilde{Z}_{k}\right)$ of functions on $N$ is regular implies that such a surface does exist and can easily be constructed.

Indeed, this regularity implies that the common level surfaces $\tilde{Z}^{-1}(z) \subset N$ of the functions in $\tilde{Z}$ are smooth surfaces of codimension $k$ in $N$. Consider a $k$-dimensional surface $\Gamma^{\prime} \subset N$ passing through the point $m$ and having the following property. The tangent subspace to $\Gamma^{\prime}$ at the point $m, T_{m} \Gamma^{\prime}$, must be transversal in $T_{m} N$ to the tangent subspace $T_{m}\left(\tilde{Z}^{-1}(z)\right)$ to the level surface $\tilde{Z}^{-1}(z) \subset N$ containing this point. Then in a sufficiently small neighbourhood of the point $m$ the surface $\Gamma^{\prime}$ has the required property that $\operatorname{rank}\left(\left.Z\right|_{\Gamma}\right)_{*}=k$. We choose as $\Gamma$ the intersection of the surface $\Gamma^{\prime}$ with this small neighbourhood of the point $m$. It is clear that the surface $\Gamma$ will then be transversal in the whole of $M$ to the common level surfaces $Z^{-1}(z)$; otherwise the restriction $\left.Z\right|_{\Gamma}$ would not be regular on $\Gamma$.

Consider the subspaces

$$
T_{m} \Gamma, \quad T_{m} \Lambda_{m} \text { and } T_{m}\left(Z^{-1}(z)\right),
$$

tangent at the point $m$, respectively, to $\Gamma$, to the leaf $\Lambda_{m} \ni m$ of this pseudo-Hamiltonian foliation $\Xi=\Xi_{N}(Z)$, and to the common level surface $Z^{-1}(z)$ passing through this point $m$. We claim that the direct sum $T_{m} \Gamma \oplus T_{m} \Lambda_{m}$ is a $2 k$-dimensional symplectic subspace of the space $T_{m} M$.

As the surfaces $\Gamma$ and $Z^{-1}(z) \ni m$ are transversal in $M$, thus $T_{m} \Gamma \cap T_{m}\left(Z^{-1}(z)\right)=\{0\}$. We have $T_{m} \Lambda_{m}=\mathcal{J}_{m} d Z$; from this, using Lemma 6.1.2 we obtain that the subspace $T_{m} \Lambda_{m}$ is isotropic and is the skew-orthogonal complement of $T_{m}\left(Z^{-1}(z)\right)$ in $T_{m} M$. It follows from these facts that the three subspaces in (13) can be taken for $R, Q$, and $P$, respectively, in Lemma 6.1.1. Applying this lemma we obtain that the $k$-dimensional subspaces $T_{m} \Gamma$ and $T_{m} \Lambda_{m}$ intersect only in zero, $0 \in T_{m} M$, and $T_{m} \Gamma \oplus T_{m} \Lambda_{m}$ is indeed a $2 k$-dimensional symplectic subspace of $T_{m} M$.

To construct $S$ (the surface required in Definition 3.4.2) we consider a sufficiently small neighbourhood $U$ of the point $m$ in $N$ and restrict the foliation $\Xi=\Xi_{N}(Z)$ to this neighbourhood. Let $\Lambda^{\prime}$ denote the leaves of this restricted foliation. For $S$ we take the union of the leaves $\Lambda^{\prime}$ intersecting $\Gamma$, that is, $S:=\bigcup_{p \in \Gamma} \Lambda_{p}^{\prime}$. Then $\Lambda_{m} \cap U \subset S \subseteq N$. Since the subspaces $T_{m} \Gamma$ and $T_{m} \Lambda_{m}$ intersect only in the zero vector, it follows from this construction that $S$ is a $2 k$-dimensional submanifold of the manifold $N$, and $T_{m} S=$ $T_{m} \Gamma \oplus T_{m} \Lambda_{m}$. Since $T_{m} \Gamma \oplus T_{m} \Lambda_{m}$ is symplectic, it follows that the entire submanifold $S$ is symplectic in $M$, that is, the 2 -form $\left.\omega^{2}\right|_{S}$ is nondegenerate. Thus, the fibration $\Xi$ is quasi-Lagrangian (see Definition 3.4.2) and therefore c) $\Longrightarrow \mathrm{b}$ ).

We now prove that b) $\Longrightarrow \mathrm{c}$ ); that is, if the foliation $\Xi=\Xi_{N}(Z)$ is quasi-Lagrangian, then $Z: \operatorname{rank}\left(\left.Z\right|_{N}\right)_{*}=k$ is internally regular. To do this, we use the fact that $\Xi$ is quasiLagrangian to construct a symplectic submanifold $S$ consisting of pieces of the leaves of the pseudo-Hamiltonian foliation $\Xi$ of the submanifold $N$ through any point $m \in N$. By hypothesis, $Z$ is regular on $N$ and therefore also on $S$. This fact and Lemma 3.5.1 imply that $\left.Z\right|_{S}$ is also regular on $S$ and, a fortiori, $\tilde{Z}=\left.Z\right|_{N}$ is regular on $S$, that is, $\operatorname{rank} \tilde{Z}_{*}(p)=k$ for all $p \in S$. Since $m$ is any point in $N$, the set $\tilde{Z}$ is regular on the whole of $N$.

Taking into account that $b) \Longrightarrow c$ ) we see that to prove that b) $\Longrightarrow d$ ), that is, that if $\Xi=\Xi_{N}(Z)$ is quasi-Lagrangian then this pseudo-Hamiltonian foliation is in fact Hamiltonian, it is sufficient to show that the vector fields $J d Z_{1}, \ldots, J d Z_{k}$ are pairwise commutative on $N$; see Definitions 3.2.2 and 3.2.3. For that it is sufficient to show that these fields commute on each leaf $\Lambda^{0} \subseteq N$ of the foliation $\Xi=\Xi_{N}(Z)$. Actually, it is 
sufficient to show that these fields are pairwise commutative on $\Lambda^{0}$ in some neighbourhood $U$ of any point $m \in \Lambda^{0}$, that is, in the intersection $\Lambda^{0} \cap U$.

As above, let $S \ni m$ be a $2 k$-dimensional symplectic submanifold $S \subseteq N$ composed of pieces of the leaves $\Lambda$ of the foliation $\Xi$. It is clear that the restriction to $S$ of the pseudo-Hamiltonian foliation $\Xi=\Xi_{N}(Z)$ of the submanifold $N$ is itself a pseudoHamiltonian foliation of the submanifold $S$ defined by the same set $Z$; see Definition 3.2.3. From this fact and Corollary 3.3.1 we obtain that this restricted foliation $\Xi_{S}(Z)$ of the symplectic submanifold $S$ is Hamiltonian. Consequently, the vector fields $J d Z_{1}, \ldots, J d Z_{k}$ are pairwise commutative on $S$ and therefore also on the intersection $\Lambda^{0} \cap S=\Lambda^{0} \cap U$, since these fields are tangent to the leaves $\Lambda$.

The implication d) $\Longrightarrow$ c) obviously follows from Definition 3.2.2 of a Hamiltonian foliation. Thus, a) $\Longleftrightarrow \mathrm{c}) \Longleftrightarrow \mathrm{b}) \Longrightarrow \mathrm{d}$ ) $\Longrightarrow \mathrm{c}$ ). The proof of Proposition 3.4.1 A) is complete.

We will now prove Proposition 3.4.1B), that is, that condition f) of commutativity:

$$
\left.[J d H, J d Z]\right|_{N}=0
$$

in the list of conditions a), b), c), d), e), f) of the first definition in the set of Definitions 3.2.4 is redundant. First, we observe that in condition e), the fact that $(H, Z)$ is critical on $N$ and that the operator $J_{m}$ is an isomorphism imply that at each point $m \in N$ the vector $J_{m} d H$ is a linear combination of the vectors $J_{m} d Z_{1}, \ldots, J_{m} d Z_{k}$. Therefore to prove Proposition 3.4.1 B), that is, to derive condition $\mathrm{f}$ ), it is sufficient to show that for any point $m_{0} \in N$ there exists a neighbourhood $U$ of this point in $N$ such that $\left.[J d H, J d Z]\right|_{\Lambda^{0} \cap U}=0$, where $\Lambda^{0} \subset N$ is the leaf of our Hamiltonian foliation $\Xi=\Xi_{N}(Z)$ containing the point $m_{0}$.

Now, the foliation is quasi-Lagrangian and we can construct the symplectic $2 k$-dimensional submanifold $S \subseteq N$ through $\Lambda^{0} \cap U$, composed of the intersections of the leaves $\Lambda$ of this foliation with $U$. When we derived the implication b) $\Longrightarrow$ d) in the proof of Proposition 3.4.1 A), we showed that under these hypotheses the restricted foliation $\Xi_{S}(Z)$ of the symplectic submanifold $S$ is Hamiltonian. This fact with the criticality of the set $(H, Z)$ on $N$ implies that Definitions 3.2 .4 a)-e) hold on $S$, that is, these conditions are satisfied if we replace $N$ by $S$.

In other words, our system satisfies all the conditions of Definition 3.2.4 imposed on a system having the Hamiltonian-sliding symmetries $Z=\left(Z_{1}, \ldots, Z_{k}\right)$ on $S$, except for condition f). This, the fact that $S$ is symplectic and Proposition 3.3.4 C) imply that condition f) is also satisfied on $S$, that is, $[J d H, J d Z]=0$ on the whole of $S$, and therefore also on the surface $\Lambda^{0} \cap U$, since the fields $J d H, J d Z_{1}, \ldots, J d Z_{k}$ are tangent to it. But $m_{0} \in N$ is arbitrary, whence Proposition 3.4.1 B) follows.

6.3. Proof of Propositions 4.3.2, 4.3.1 A) and B). To prove Proposition 4.3.2 we first show that a set $Z$ that satisfies the main conditions of this proposition, that is, all the conditions apart from a)-d) which we want to show are all equivalent, defines a pseudo-Hamiltonian fibration $\Xi_{N}(Z)$ of the submanifold $N$.

Indeed, using Remark 3.2.1 we can see that in order to prove that $\Xi_{N}(Z)$ is pseudoHamiltonian it is sufficient to show that it satisfies conditions a), $b^{\prime}$ ), and d) of Definition 3.2.3, and that the distribution $\mathcal{J}_{N} d Z$ defines a fibration on $N$. But the tangency condition a) on $N$ and condition $\mathrm{b}^{\prime}$ ) that $Z$ is regular on $N$ are obviously satisfied, while the isotropy condition d) follows from Proposition 3.3.3, as by hypothesis $\left.\{Z, Z\}\right|_{N}=0$. The Frobenius integrability of the field of planes $\mathcal{J}_{N} d Z$ and the fibration of $N$ by the leaves of the foliation defined by this field follow from the hypothesis that $d G(J d Z)=0$, the equality $k+r=\operatorname{dim} N$, and formula (4), using the regularity of the sets $Z$ and $G$. 
Furthermore, the fibres of this pseudo-Hamiltonian fibration $\Xi_{N}(Z)$ obviously coincide with the fibres of the fibration $\Xi_{N G}$ corresponding to the map $G: N \rightarrow \mathbf{R}^{r}$.

We further observe that the additional conditions a), b), c), d) of Proposition 4.3.2, which we are proving equivalent, are completely equivalent to the corresponding conditions of Proposition 3.4.1 A) for the case of fibrations (denoted in the same way and in the same order). In fact, these four conditions coincide with the same conditions in Proposition 3.4.1 A), but they are stated for the special situation of fibrations considered in Proposition 4.3.2, rather than for foliations. The fact that, as we have shown, $\Xi_{N}(Z)$ is pseudo-Hamiltonian, along with Proposition 3.4.1 A) proves Proposition 4.3.2.

We now prove Proposition 4.3.1 A). We have that $\Phi=(Z, \mathcal{F})$ is a set of functions Hamiltonian-integrable on $N \subseteq M$ (see Definition 4.2.1), and we need to show that Definitions 3.2.2 a)-d) hold for fibrations. This can easily be done using Proposition 4.3.2, but we will do it directly. The isotropy of the foliation defined by the vector fields $J d Z_{1}, \ldots, J d Z_{k}$ of the set $J d Z$ follows from the involutivity condition in Definition 4.2.1 b), $\left.\{Z, Z\}\right|_{N}=0$, and formula (4); see also Proposition 3.3.3.

Again using Definition 4.2.1b), $\left.\{Z, Z\}\right|_{N}=0$, and (4) we find that $d \tilde{Z}(J d Z)=0$ on $N$, where $\tilde{Z}:=\left.Z\right|_{N}$. From this fact and Definition $\left.4.2 .1 \mathrm{~d}\right), d \mathcal{F}(J d Z)=0$, we obtain that $d F(J d Z)=0$ on $N$, where $F:=(\tilde{Z}, \mathcal{F})$. Now Definition $4.2 .1 \mathrm{e})$, which says that $F$ is regular on $N$, implies the regularity of $\tilde{Z}$ and therefore also of $Z$ on $N$. This fact and the equality $d F(J d Z)=0$, as well as conditions a), $2 k+l=\operatorname{dim} N$, and e), the regularity of $F$ on $N$, imply that the $k$ fields in the set $J d Z$ define a foliation of $N$, which is a fibration of the submanifold $N$ with fibring map $F: N \rightarrow \mathbf{R}^{k+l}$. According to Corollary 3.4.1, in the case of fibrations, condition c) of pairwise commutativity follows from the other conditions of Definition 3.2.2 of a Hamiltonian fibration, which we have verified. Thus, assertion 4.3.1 A) is proved in one direction.

We now prove it in the other direction. The assertion that the dimension $\operatorname{dim} N \geq 2 k$ and that the submanifold $N$ for $\operatorname{dim} N=2 k$ is symplectic follow from the fact that a Hamiltonian foliation is quasi-Lagrangian; see Proposition 3.4.1 A), which we proved in $\S 6.2$. The main part of the converse assertion is proved as follows.

Proposition 3.3.3 and the isotropy of the given Hamiltonian fibration $\Xi_{N}(Z)$ of the submanifold $N$ defined by the set $Z$ imply that $\left.\{Z, Z\}\right|_{N}=0$, that is, Definition $4.2 .1 \mathrm{~b}$ ) holds. From this fact, using (4), we obtain that $d Z(J d Z)=0$ on $N$; see also Proposition 3.3.3. Consequently, the functions $Z_{1}, \ldots, Z_{k}$ are constant on the connected components $\Lambda$ of the inverse images $\psi^{-1}(b) \subset N$ of points $b \in B$ under the map $\psi: N \rightarrow B$ defining the fibration of the submanifold $N$. We consider an arbitrary connected compact set $K \subseteq \psi^{-1}\left(b_{0}\right)$ contained in the inverse image $\psi^{-1}\left(b_{0}\right)$ of some point $b_{0} \in B$. Using the regularity of the map $\psi$ (see Definition 3.2.1) one can easily show that there exists a neighbourhood $U$ in $N$ of this compact set having the following properties. The nonempty inverse images $\psi_{U}^{-1}(b)$ of every point $b \in B$ under the map $\psi_{U}:=\left.\psi\right|_{U}$ are connected submanifolds of $U$. Furthermore, $\psi(U)$ is a neighbourhood of the point $b_{0}$ in $B$. Here $K \subset U \subseteq N$ and $\psi_{U}^{-1}(b) \subset U$.

Using this fact we construct a set $\mathcal{F}$ of functions on $U$. The restrictions of the functions in the set $Z$ to the inverse images $\psi_{U}^{-1}(b)$ are constant because they are connected. This fact and the regularity of $\psi$ imply the existence of a neighbourhood $\mathcal{V}$ of the point $b_{0} \in B$ such that the composites $\left.\left.Z_{i}\right|_{U} \circ \psi_{U}^{-1}\right|_{\mathcal{V}}$ define smooth functions $z_{i}: \mathcal{V} \rightarrow \mathbf{R}, i=1, \ldots, k$. As $\left.Z\right|_{N}$ is regular on $N$, the functions $z_{1}, \ldots, z_{k}$ are functionally independent in some neighbourhood $\mathcal{V}$ of the point $b_{0}$ in $B$. We complement these $k$ functions by functions $f_{1}, \ldots, f_{l}$ to obtain a set of smooth coordinates in $\mathcal{V}$. (In fact, these neighbourhoods $\mathcal{V}$ can become smaller at each step of the argument.) We set $\mathcal{F}_{j}:=f_{j} \circ \psi_{U}, j=1, \ldots, l$, 
and $\tilde{Z}_{i}:=\left.Z_{i}\right|_{U}$. Clearly, then $\tilde{Z}_{i}=z_{i} \circ \psi_{U}$ for all $i=1, \ldots, k$, and the functions in the set $\mathcal{F}=\left(\mathcal{F}_{1}, \ldots, \mathcal{F}_{l}\right)$ are smooth.

It is also clear that the set $F=(\tilde{Z}, \mathcal{F})$ of $k+l$ functions on $U$ defines the map $\psi_{U}$ in the local coordinates $y=(z, f)$ defined in $\mathcal{V} \subseteq B$. This fact and $\psi$ being regular imply that $F$ is regular on $U$. Since $\psi$ is the fibring map, we have $\operatorname{dim} N=k+(k+l)=2 k+l$ and $d F(J d Z)=0$ on $U$ and therefore also $d \mathcal{F}(J d Z)=0$ on $U$. Thus, all five conditions a $)-\mathrm{e})$ in Definition 4.2.1 for the mixed set $\Phi=(Z, \mathcal{F})$ to be Hamiltonian-integrable on $U \subseteq N$ are satisfied. The proof of Assertion 4.3.1 A) is complete.

Assertion 4.3.1 B) is an analogue of Proposition 4.3.1 A) for systems instead of fibrations. It clearly follows from this proposition and Definitions 4.2.2 and 3.2.4 of systems that are Hamiltonian-integrable on a submanifold of the phase space, together with Proposition 3.4.1 B), which states that in Definition 3.2.4 condition $\mathrm{f}$ ), requiring that the field $J d H$ commute with the fields in the set $J d Z$ on this submanifold, is redundant.

6.4. Proof of Theorem 5.5.1 A). First of all we note that the fibres-tori $\Lambda$ form a locally trivial fibration of the submanifold $\sigma \subseteq N \cap Z^{-1}(z)$. This follows from the fact that such tori $\Lambda$ form a locally trivial fibration of the submanifold $N$ and that the submanifold $\sigma$ is smooth and has the completeness property.

It follows from Definition 5.2.1 of circular action functions that to prove the theorem, that is, to prove that $\left.I\right|_{\sigma}=$ const it is sufficient to do the following. First we must take any two points $m_{1}$ and $m_{2}$ on $\sigma$ sufficiently close to each other and consider the fibres-tori $\Lambda^{1} \ni m_{1}$ and $\Lambda^{2} \ni m_{2}$ of this fibration $\Xi_{N}(J d Z)$ of the submanifold $N$ containing these points. Then we must fix any $i=1, \ldots, k$ and consider some closed curves $\delta_{i 1} \subseteq \Lambda^{1}$ and $\delta_{i 2} \subseteq \Lambda^{2}$ lying on these tori and corresponding to the $i$ th basis cycle in the fundamental groups of these tori with compatible bases in these groups. Then we must stretch a 2-dimensional film $\Pi_{i} \subseteq \sigma$ connecting the curves $\delta_{i 1}$ and $\delta_{i 2}$ and show that

$$
\iint_{\Pi_{i}} \omega^{2}=0
$$

where $\omega^{2}$ is the symplectic form on $M$.

We construct the film $\Pi=\Pi_{i}$ as follows. We choose an arbitrary smooth curve $\alpha \subset \sigma$ that connects the points $m_{1}$ and $m_{2}$ and intersects the fibre-torus $\Lambda_{m} \ni m$ of the fibration $\Xi_{N}(J d Z)$ at each point $m \in \alpha$ at a nonzero angle. It is clear that such a curve always exists. Then through each point $m \in \alpha$ we construct a closed curve $\delta_{i m} \subseteq \Lambda_{m}$ representing the $i$ th basis element of the fundamental group $\pi_{1}\left(\Lambda_{m}\right)$ of the torus $\Lambda_{m}$. It is easy to see that these curves $\delta_{i m}$ can be chosen to be such that the film $\Pi=\bigcup_{m \in \alpha} \delta_{i m}$ swept out by them is a smooth 2-dimensional surface. The boundary of this film contained in $\sigma$ is formed by the cycles $\delta_{i 1}:=\delta_{i m_{1}}$ and $\delta_{i 2}:=\delta_{i m_{2}}$. As the tori $\Lambda$ give a locally trivial fibration of our surface $\sigma$ all these constructions can indeed be carried out.

We claim that the film $\Pi$ constructed above is isotropic, that is, $\left.\omega^{2}\right|_{\Pi}=0$, which will prove Theorem 5.5.1 A). We take any point $m \in \Pi$; then it follows from the construction of $\Pi$ that there exists a nonzero vector $\eta \in T_{m} \Pi$ tangent to the torus $\Lambda_{m}$ and passing through this point. Consequently, the vector $\eta$ belongs to the linear span $\mathcal{J}_{m} d Z$ of the vectors of the fields $J d Z_{1}, \ldots, J d Z_{k}$ at this point. On the other hand, $\Pi \subseteq \sigma \subseteq Z^{-1}(z)$; hence it follows from Lemma 6.1.2 A) that all the tangent vectors to $\Pi$, that is, all the vectors in $T_{m} \Pi$, are skew-orthogonal to the vector $\eta$. This implies that $T_{m} \Pi$ has a basis of two vectors $\eta$ and $\xi$ that are skew-orthogonal to each other. Thus, the form $\left.\omega^{2}\right|_{\Pi}$ vanishes at the point $m \in \Pi$ and therefore also on the whole of $\Pi$. This proves that the integrals $\iint_{\Pi_{i}} \omega^{2}$ are equal to zero; thus Theorem 5.5.1 A) is proved. 
6.5. Proof of Theorem 5.5.1 B). The fact that each fibre $\Lambda$ of the fibration of either of the types considered in the theorem is diffeomorphic to the $k$-dimensional torus follows from Proposition 4.3.1 A), Definition 3.2.2, and Proposition 2.2.1.

We also note that the remaining assertions of this theorem are semilocal in the sense that they guarantee certain properties only in some sufficiently small neighbourhood of any fibre $\Lambda^{0} \subset N$ in $N$. But $\Lambda^{0}$ is diffeomorphic to a torus, that is, it is a compact set. From this and Proposition 4.3.1B) we obtain the following fact. In a sufficiently small neighbourhood in $M$ of each fibre $\Lambda^{0} \subset N$, systems Hamiltonian-torically $Z$ integrable on $N \subseteq M$ in the sense of Definition 3.2.4 have just the same structure as systems Hamiltonian-torically $\Phi$-integrable on $N$ in the sense of Definition 4.2.2, where $\Phi=(Z, \mathcal{F})$. More precisely, if the restriction of the system to such a neighbourhood in $M$ satisfies one of these two definitions, then it also satisfies the other one with the same set $Z$. Furthermore, the fibres of the fibrations $\Xi_{N}(Z)$ and $\Xi_{N F}$ corresponding to these two definitions coincide; cf. Remark 5.5.4.

Using this fact, in what follows by the fibration $\Xi$ of a submanifold $N$ we shall mean, depending on the situation, either the fibration $\Xi_{N}(Z)$ defined by the vector fields in the set $J d Z$ (see Definition 3.2.1) or the fibration $\Xi_{N F}$ into the connected components $\Lambda$ of the inverse images of points under the map $F: N \rightarrow \mathbf{R}^{k+l}$, where $F=\left(\left.Z\right|_{N}, \mathcal{F}\right.$ ) (see Definition 4.2.1). Taking this into account, from Definition 3.2.2 and Proposition 2.2.1 we obtain that the fibration $\Xi$ of the submanifold $N$ into the tori $\Lambda$ is locally trivial; see also Definition 3.2.1 of a fibration and Remark 3.2.0. The rest of the proof is based on the following lemma.

Lemma 6.5.1. Suppose that all the conditions of Theorem 5.5.1B) hold. Then for each fibre $\Lambda^{0}$ of the toric fibration $\Xi$ of the submanifold $N \subseteq M$ under consideration there exists a symplectic $2 k$-dimensional submanifold $S$ containing $\Lambda^{0}$ and having the following three properties.

A) This submanifold $S \supset \Lambda^{0}$ is contained in $N: S \subseteq N$, and moreover, it is composed of whole fibres $\Lambda$ of the fibration $\Xi$. In other words, for any point $m \in S$ the fibre-torus $\Lambda_{m}$ passing through $m$ is entirely contained in $S: \Lambda_{m} \subset S$. Furthermore, in some neighbourhood of $S$ in $N$ the set $I=\left(I_{1}, \ldots, I_{k}\right)$ of circular action functions is defined on $N$ corresponding to the Hamiltonian fibration $\Xi$ of the submanifold $N$ into the tori $\Lambda$; see Definition 5.2.1.

B) Set $\bar{H}:=\left.H\right|_{S}$; also let $\bar{Z}:=\left.Z\right|_{S}$ and $\bar{I}:=\left.I\right|_{S}$ denote the sets obtained by restricting the functions in the sets $Z$ and $I$ respectively to $S$. Then the field $J d H$ of the original system is tangent to the submanifold $S: J_{m} d H \in T_{m} S$ for any point $m \in S$. Furthermore, the restriction of this system to $S$ is the system with Hamiltonian $\bar{H}$ that is Hamiltonian-torically $\bar{Z}$-integrable on the whole of $S$. In addition, $\bar{H}$ is a function only of the functions in the set $\bar{I}: \bar{H}=\bar{H}(\bar{I})$. The tori-fibres $\Lambda \subset S$ of the fibration $\Xi$ lying on $S$ coincide with the $k$-dimensional tori $\{\bar{I}=$ const $\} \subset S$ and are invariant under the local flow of the original system with Hamiltonian $H$. The motion on these tori is conditionally periodic with frequencies $\left(\omega_{1}, \ldots, \omega_{k}\right)=\omega$ such that $\omega=\omega(\bar{I})=\frac{\partial \bar{H}}{\partial \bar{I}}(\bar{I})$.

C) The action variables $\bar{I}$ and the functions in the set $\bar{Z}$ can be expressed as functions of each other: $\bar{I}=\bar{I}(\bar{Z})$ and $\bar{Z}=\bar{Z}(\bar{I})$.

Proof of Lemma 6.5.1. To prove assertion A) we first observe that as the fibration $\Xi$ of the submanifold $N$ into the tori $\Lambda$ is locally trivial, applying Proposition 5.2.1 we obtain the following. In some neighbourhood of each fixed torus $\Lambda^{0}$ of this fibration it is possible to construct the set $I$ of circular action functions corresponding to the fibration $\Xi$. 
To construct the submanifold $S \supset \Lambda^{0}$ we consider any point $m_{0} \in \Lambda^{0}$. As the fibration $\Xi$ is quasi-Lagrangian (see Propositions 3.4.1 A) and 4.3.2) we can construct a $2 k$-dimensional symplectic submanifold $S_{0}$ through this point such that $J_{m} d Z_{i} \in T_{m} S_{0}$ at each point $m \in S_{0}$ and for any $i=1, \ldots, k$. We assume that $S_{0}$ is contained in a sufficiently small neighbourhood of the point $m_{0}$.

For the submanifold $S$ containing $\Lambda^{0}$ and composed of whole leaves $\Lambda$ of the fibration $\Xi$ we take $S=\bigcup_{m \in S_{0}} \Lambda_{m}$, where $\Lambda_{m} \ni m$. As the fibration $\Xi$ of the submanifold $N$ into the tori $\Lambda$ is locally trivial we obtain that $S$ is a $2 k$-dimensional submanifold of $N$ such that its intersection $S \cap V$ with a small neighbourhood $V$ of the point $m_{0}$ coincides with $S_{0}$. Furthermore, for sufficiently small $S_{0}$ the submanifold $S=S\left(S_{0}\right)$ is trivially fibred by these isotropic tori $\Lambda$ and is contained in a small neighbourhood of the torus $\Lambda^{0}$.

It remains to prove that the submanifold $S$ is symplectic, that is, that the 2 -form $\left.\omega^{2}\right|_{S}$ is nondegenerate. Consider the local phase flows $\left\{g_{Z_{i}}^{t}, t \in \mathbf{R}\right\}$ of the vector fields $J d Z_{i}, i=1, \ldots, k$. As is well known (see, for example, [1]), the phase flow of a Hamiltonian system preserves the symplectic structure $\omega^{2}$ on $M$ and therefore also preserves the restriction $\left.\omega^{2}\right|_{R}$ of this form to any invariant submanifold $R \subset M$ of the system. Since the fields $J d Z_{1}, \ldots, J d Z_{k}$ are tangent to $S$, this implies that the corresponding symplectomorphisms $g_{Z_{i}}^{t}, i=1, \ldots, k$, preserve the 2 -form $\left.\omega^{2}\right|_{S}$.

According to Definition $3.2 .2 \mathrm{c}$ ), the fields $J d Z_{1}, \ldots, J d Z_{k}$ are pairwise commutative on $N$ and therefore also on $S$. Consequently, the restrictions of these fields to $S$ form a basis of generators of an abelian group, which is isomorphic to $\mathbf{R}^{k}$. It follows from the definition of the fibration $\Xi=\Xi_{N}(Z)$ (see also Definition 3.2.2 c)) that the fibres $\Lambda$ of this fibration contained in $S$ coincide with orbits of this group. But the restriction of the 2 -form $\omega^{2}$ to $S_{0}$ is nondegenerate, and from the construction of $S$ for any point $m \in S$, the fibre $\Lambda_{m}$ of the fibration $\Xi$ passing through this point intersects $S_{0}$. This fact and the invariance of $\left.\omega^{2}\right|_{S}$ under the transformations $g_{Z_{i}}^{t}, i=1, \ldots, k$, imply that this form is nondegenerate on the whole of $S$.

Proof of assertion B). Assertion A) on the properties of the submanifold $S$ and the fact that our system is Hamiltonian-torically $Z$-integrable on $N$ obviously imply that the system is weakly Hamiltonian-torically $Z$-integrable on the submanifold $S \subseteq N \subseteq M$; see Definitions 3.2.4, 3.2.2, and 3.2.3. From this fact, since $S \subseteq M$ is symplectic, using Corollary 3.3.1 we find that the system is Hamiltonian-torically $Z$-integrable on $S$. Consequently, we can apply Proposition 5.1.1, which implies the following. The vector field $J d H$ of the original system is tangent to the submanifold $S$. In addition, the restriction of this field to $S$ is the Hamiltonian system on $\left(S, \bar{\omega}^{2}\right)$ with Hamiltonian function $\bar{H}$, where $\bar{\omega}^{2}:=\left.\omega^{2}\right|_{S}$. Furthermore, in some canonical coordinates $(J, \psi \bmod 2 \pi)$ defined on $S$ the Hamiltonian $\bar{H}$ has the form $\bar{H}=\bar{H}(J)$, while $J=J(\bar{Z})$ and $\bar{Z}=\bar{Z}(J)$. The variables $J$ are circular action functions; hence we can take $J=\bar{I}$; see Proposition 5.2.1. The fact that the motion on the tori $\Lambda=\{\bar{I}=$ const $\} \subset S$ is conditionally periodic and the required form of the frequency $\omega$ of this motion follow from Proposition 5.1.1 B). This proves 6.5.1 B). Now, 6.5.1 C) follows from Proposition 5.1.1 A). Thus the proof of Lemma 6.5.1 is complete.

The completion of the proof of Theorem 5.5.1 B). In the lemma we have just proved, we have shown that the Hamiltonian function $H$, the set $\omega$ of frequencies of the conditionally periodic motion, and the set $I$ of action functions depend only on the functions in the set $Z$, but only on the surface $S \subseteq N, \Lambda^{0} \subset S$. The same is also true of the other functional dependencies we need. If we can prove these in the whole of the neighbourhood $U$ in $N$ of an arbitrary fixed torus $\Lambda^{0}, \Lambda^{0} \subset U \subseteq N \subseteq M$, then the proof of Theorem 5.5.1B) will be complete. The extension of the required relations between $\tilde{Z}=\left.Z\right|_{U}, I$, $\omega$, and $\tilde{H}=\left.H\right|_{U}$ from $S$ to the entire neighbourhood $U$ in $N$ of the torus $\Lambda^{0}$ can be easily 
obtained from the fact that $I$ and $\tilde{H}$ depend only on $\tilde{Z}$ in the entire $U \subseteq N$. A more detailed argument is as follows.

We rewrite the dependencies obtained on $S$ in the preceding lemma using different letters to denote the maps connecting the sets of functions under consideration:

$$
\bar{I}=\beta(\bar{Z}), \quad \bar{Z}=\beta^{-1}(\bar{I}), \quad \bar{H}=\nu(\bar{I}), \quad \bar{\omega}=\Delta \nu(\bar{I}),
$$

where $\Delta \nu$ is the vector of partial derivatives of a function $\nu$ of $k$ variables, and $\bar{\omega}$ is the set of frequencies on $S$. We emphasize that all these maps are smooth. Using the assumption in our theorem that $d H=\sum_{i=1}^{k} \lambda_{i} d Z_{i}$ on $N$, the fact that the torus is connected, that the fibration of $N$ by the tori $\Lambda \subset Z^{-1}(z)$ is locally trivial and the regularity of the set $\tilde{Z}$ we obtain the following assertion. In some neighbourhood $U$ of the torus $\Lambda_{0}$ in $N$ we have $\tilde{H}=\kappa(\tilde{Z})$, where $\kappa$ is some smooth function. Using the same facts we obtain from Theorem 5.5.1 A) that $I=\mu(\tilde{Z})$ in $U$.

From the relations $I=\mu(\tilde{Z}), \bar{I}=\beta(\bar{Z})$, and $\bar{I}=\left.I\right|_{S}, \bar{Z}=\left.\tilde{Z}\right|_{S}$, and as the functions in the set $\bar{Z}$ are functionally independent, we obtain $\beta=\mu$. As $\beta^{-1}$ exists and is smooth, so is $\mu^{-1}=\beta^{-1}$. Consequently, $\tilde{Z}=\mu^{-1}(I)$ in the entire neighbourhood $U$ of the torus $\Lambda^{0}$ in $N$. Thus, $\tilde{H}=\rho(I)$ on $U$, where $\rho=\kappa \circ \mu^{-1}$. Using the same arguments as we used to prove $\beta=\mu$ we obtain $\nu=\rho$.

Consequently, for each torus $\Lambda \subset S=S\left(\Lambda^{0}\right)$, including the torus $\Lambda^{0}$, we have $\omega=$ $\triangle \rho(I)$, where $I=I(\Lambda)$ and $\omega$ is the set of frequencies on this torus. Since the fibre $\Lambda^{0} \subset N$ is arbitrary, this argument is valid for any fibre $\Lambda \subset U$ if it is chosen for $\Lambda^{0}$. Thus, for any $\Lambda \subset U$ we have $\omega(I)=\triangle \rho(I)$, that is, $\omega(I)=\frac{\partial \tilde{H}}{\partial I}(I)$, where $I=I(\Lambda)$. Thus, all the assertions of Theorem 5.5.1 B) are proved.

\section{Equilibrium SUbManifoldS}

This section largely deals with how to find integrability submanifolds, especially those that are toric. In $\S 2.3$ we looked at this for systems of general form, while here we study it for Hamiltonian systems. We consider the case where some centred set of functions $\Phi=(Z, \mathcal{F})$ is known, that is, a set of functions defined on the entire phase space $M$ and such that $\{Z, \Phi\}=0$ on $M$; see Definition 4.1.2. We also consider more general situations. We are interested in general methods for finding the submanifolds $N \subseteq M$ on which a given system is weakly or strongly Hamiltonian- $Z$-integrable.

But to start with, in $\S \S 7.1$ and 7.2 , we consider the question of where the centred sets $\Phi$ themselves come from. As we already noted at the end of $\S 1.1$, local Lie groups with Poisson action on symplectic manifolds $M$ give one of the main and simplest sources of such sets, $\Phi=(Z, \mathcal{F})$. In $\S 7.1$ using our terminology, we remind the reader how a centred set $\Phi=(Z, \mathcal{F})$ is constructed with respect to any such Lie group. In $\S 7.2$ we do the same in the more general situation of a set of functions $F$ on $M$ that is Poisson closed. In $\S 7.3$ we consider the case where the set $F$ is a set of integrals of some system. We discuss how in this and more general situations one can find the maximal submanifold $N$ of local $Z$-integrability of this system, specifying it by the sliding condition. We study some properties of these $N$.

In $\S 7.4$ we shall describe a method for finding the "equilibrium submanifolds". The trajectories lying on them generalize the well-known "relative equilibrium positions"; see, for example, 33. This method is applied when $Z$ is not a set of integrals of the system, that is, when $\{H, Z\} \not \equiv 0$ on $M$. Roughly speaking, to find an integrability submanifold $N$ we consider the system of equations corresponding to the sliding condition and then apply the operator $\{\bullet, Z\}$ to the right-hand sides of these equations "repeatedly"; each time the result is equated to zero. The integrability which is obtained on such equilibrium submanifolds $N$ is, generally speaking, merely local. In $\S 7.5$ we give an informal but more 
effective algorithm based on the contents of $\S 7.4$, which allows one, in a larger number of cases, to find the submanifolds on which the system is globally $Z$-integrable.

7.1. Construction of the centred set of functions induced by a Poisson action of a local Lie group. Recall that by a local Lie group $G$ acting on a manifold $M$ we mean some Lie algebra $\gamma$ of vector fields on $M$. The action of the corresponding local Lie group $G=G(\gamma)$ on the symplectic manifold $M$ is said to be Poisson if the following holds. First, all the vector fields in $\gamma$ are globally Hamiltonian on $M$. Second, the resulting map $\gamma \rightarrow C^{\infty}(M)$ of elements $a$ in $\gamma$ to the corresponding Hamiltonians $F_{a}$ defines a homomorphism of the Lie algebra $\gamma$ into the Lie algebra of functions on $M$ with the Poisson bracket as the operation; see [1, 3].

This homomorphism $a \mapsto F_{a}$ defines the so-called momentum map $F: M \rightarrow \gamma^{*}$ from $M$ into the Lie coalgebra $\gamma^{*}$ of the given group $G$. Indeed, for any point $m \in M$ there is a linear map $\gamma \rightarrow \mathbf{R}$ associating with each field $a \in \gamma$ the number $F_{a}(m)$, and such a map is an element of the coalgebra $\gamma^{*}$. In other words, $F$ is defined by the equality $\langle F(m), a\rangle=F_{a}(m)$ for any element $a \in \gamma$. We fix some basis $a_{1}, \ldots, a_{s}$ of $\gamma$ and let $F_{1}, \ldots, F_{s}$ be Hamiltonian functions on $M$ defining these vector fields: $a_{i}=J d F_{i}$. Then in the coordinates in $\gamma^{*}$ corresponding to this basis in $\gamma$ the momentum map is given by the set of functions $F=\left(F_{1}, \ldots, F_{s}\right)$, so that $F: M \rightarrow \mathbf{R}^{s} \cong \gamma^{*}$.

The centring of the set $F$ is carried out as follows. Let $\xi \in \gamma^{*}$ be an arbitrary typical point, that is, lying on an orbit of the coadjoint representation that has maximum dimension. Let $z_{1}, \ldots, z_{k}$ be a complete set of functionally independent invariants $z_{i}: \gamma^{*} \rightarrow \mathbf{R}$ of the coadjoint action of the group $G$ on $\gamma^{*}$. Here local invariants are considered, that is, defined in some neighbourhood of this point. Consider the corresponding functions $Z_{i}$ on $M$, that is, the composites $Z_{i}=z_{i} \circ F$. Then each of the functions in the set $Z=\left(Z_{1}, \ldots, Z_{k}\right)$ is in involution with each of the functions in the set $F$ and therefore the functions in the set $Z$ themselves are pairwise in involution.

Indeed, the Poisson action of the group $G$ on $M$ under the momentum map is transformed into the coadjoint action of this group; see [1, 3. Since the $z_{i}$ are invariants of this action, thus $0=d Z_{i}\left(J d F_{j}\right)=\left\{Z_{i}, F_{j}\right\}$ for all $i=1, \ldots, k, j=1, \ldots, s$, where $\{$, is the Poisson bracket of functions on the symplectic manifold $\left(M, \omega^{2}\right)$. The functions in the set $Z$ are obviously functions of the set $F: Z=Z(F)$; hence, $\{Z, Z\}=0$. If the group $G$ acts locally freely, then the functions in the set $F$ are functionally independent. Then the functions in the set $Z$, as well as in the set $z$, are also functionally independent. Replacing $k$ suitable functions in the set $F$ by functions in the set $Z$ we obtain a centred set $(Z, \mathcal{F})$ of $s$ functionally independent functions that are locally functionally dependent on the functions in the set $F$.

A trivial example is provided by a Poisson action of an abelian Lie group $G$ - to such a group there obviously corresponds a set of functions $F_{1}, \ldots, F_{s}$ in involution. Conversely, to any set of functions $Z_{1}, \ldots, Z_{k}$ that are pairwise in involution there corresponds a $k$ dimensional abelian group with Poisson action diffeomorphic to $\mathbf{R}^{k}$. Consequently, the standard construction $F \rightarrow Z$ of the set $Z$ from $F$ described above can be regarded as "abelianization" of a nonabelian Lie group $G$ with Poisson action on a symplectic manifold $M: G \longrightarrow \mathcal{G}$, where $\mathcal{G} \cong \mathbf{R}^{k}$.

Note that in the case of systems of general form the corresponding transition to this one is the transition $(G, V) \longrightarrow \mathcal{G}$ described in $\S 2.3$. But in this general situation, in contrast to the Hamiltonian case, the original vector field $V$ is used directly. Furthermore, the "abelianization" $\mathcal{G} \cong \mathbf{R}^{k}$ of the original group $G$ is only constructed on a submanifold $N \subseteq M$ where $G$ is a group of sliding symmetries of the field $V$. In other words, $N$ must be invariant under the action of the group $G$, and the field $V$ must be tangent to the orbits of this group on it. 
7.2. Centring of a Poisson closed set of functions. The situation described in the preceding subsection is a special case of the following more general situation.

Definition 7.2.1. A set of functions $F=\left(F_{1}, \ldots, F_{s}\right)$ is said to be Poisson closed if the Poisson bracket of any two functions in the set is a function of the set $F:\left\{F_{i}, F_{j}\right\}=$ $f_{i j}(F), i, j=1, \ldots, s$.

In invariant terms this means that the map $F: M \rightarrow \mathbf{R}^{s}$ defined by this set is single-valued and gives a well-defined Poisson structure on $\mathbf{R}^{s}$ with respect to the symplectic structure $\omega^{2}$ on $M$; see 3. Suppose also that the rank of the square "Poisson matrix" $\{F, F\}$ of size $s \times s$ composed of the Poisson brackets $\left\{F_{i}, F_{j}\right\}$ is constant: $\operatorname{rank}\left(f_{i j}(F), i, j=1, \ldots, s\right)=$ const. In other words, the rank of the Poisson structure on $\mathbf{R}^{s}$ transferred from $\left(M, \omega^{2}\right)$ is constant (see [3]); this rank by definition coincides with $\operatorname{rank}\{F, F\}$.

In the case of a Poisson action of a local Lie group considered in the preceding subsection we have $\mathbf{R}^{s}=\gamma^{*}$ and all the functions $f_{i j}(F)$ are linear: $f_{i j}(F)=\sum_{k} c_{i j}^{k} F_{k}$, where the $c_{i j}^{k}=$ const are the so-called structure constants of the Lie algebra $\gamma$ corresponding to this group. In this case the Poisson structure on the coalgebra $\gamma^{*}$ is fixed, that is, it is defined only by the algebra $\gamma$ itself. Here the rank of this structure is maximal at all the typical points of the coalgebra $\gamma^{*}$. The latter means that these points $\xi \in \gamma^{*}$ lie on typical orbits of the coadjoint action of the group, that is, orbits of maximum dimension. This dimension coincides with the rank of the structure. Such orbits form a submanifold of zero codimension in $\gamma^{*}$ that is everywhere dense in $\gamma^{*}$. If the image of the map $F: M \rightarrow \gamma^{*}$ consists only of typical points, then $F$, the corresponding set of functions on $M$, satisfies the condition $\operatorname{rank}\{F, F\}=$ const: the rank of the Poisson matrix is constant.

In the general case, instead of a set $F=\left(F_{1}, \ldots, F_{s}\right), F: M \rightarrow \mathbf{R}^{s}$, one must consider a map $F: M \rightarrow B$, where $B$ is a manifold with the Poisson structure that is transferred from $M$ by this map. But we are only working semilocally, that is, in a neighbourhood of the inverse image $F^{-1}(b) \subset M$; therefore we simply have to consider a set of functions $F$, and correspondingly $\mathbf{R}^{s}$ instead of $B$.

Proposition 7.2.1. Suppose that the functions in the set $F=\left(F_{1}, \ldots, F_{s}\right)$ are functionally independent in a neighbourhood of each point $m \in M$, and suppose that the set is Poisson closed and the rank of the Poisson matrix $\{F, F\}$ is constant on $M$, as above. Then for each point $\xi \in \mathbf{R}^{s}$ there exists a centred set $\Phi=(Z, \mathcal{F})$ of s functions, that is, $\{Z, \Phi\}=0$, satisfying the following four conditions.

a) All the functions in the set $\Phi$ are defined in $F^{-1}(\mathcal{V})$, where $\mathcal{V} \subseteq \mathbf{R}^{s}$ is some neighbourhood of the point $\xi$ in $\mathbf{R}^{s}$.

b) The number $k$ of central functions, that is, functions that belong to the subset $Z=\left(Z_{1}, \ldots, Z_{k}\right)$, is defined by the formula $k=\operatorname{corank}\{F, F\}:=s-\operatorname{rank}\{F, F\}$.

c) The set $\Phi$ is functionally equivalent to the original set $F$, that is, in the domain $F^{-1}(\mathcal{V})$ each of these sets can be functionally expressed in terms of the other: $\Phi=\Phi(F), F=F(\Phi)$.

d) The functions in the subset $Z$ are, in fact, uniquely determined by the preceding conditions. More precisely, if $\Phi^{\prime}=\left(Z^{\prime}, \mathcal{F}^{\prime}\right)$ is another set satisfying all these three conditions a), b), c) and such that $\left\{Z^{\prime}, \Phi^{\prime}\right\}=0$, then $Z^{\prime}=Z^{\prime}(Z)$.

The functions in the set $\Phi$ are constructed as follows. In a neighbourhood of the point $\xi \in \mathbf{R}^{s}$ we choose local coordinates $(z, f)$ such that the surfaces $z=$ const specify the symplectic leaves in $\mathbf{R}^{s}$ of the Poisson structure under consideration, whose dimension coincides with $\operatorname{rank}\{F, F\}$. (In the special case of local Lie groups these leaves on $\gamma^{*}$ 
coincide with the orbits of the coadjoint action.) In other words, for the set $z$ we take the complete set of functionally independent Casimir functions. Consider the set $\Phi=(Z, \mathcal{F})$ of liftings of the functions in the set $(z, f)$ from $\mathbf{R}^{s}$ to $M$, that is, $Z:=z \circ F$ and $\mathcal{F}:=f \circ F$. It is easy to verify that this set is the required one.

It easily follows from formula (4) that $\operatorname{rank}\{F, F\}$ does not change when the set $F$ is replaced by an equivalent one. (For the geometric meaning of this rank see the proof of Lemma 7.3.1.) Therefore if the central subsets $Z$ and $Z^{\prime}$ of two sets equivalent to $F$ were functionally dependent, this would cause the rank to drop with respect to $s-k$ for some set equivalent to $F$. From this we obtain that the centred set $\Phi=(Z, \mathcal{F})$ is unique.

7.3. Finding the integrability submanifolds of the system in the presence of an involutive set of integrals. Proposition 7.3.1 C) below shows the following. Let $\Phi=(Z, \mathcal{F})$ be an arbitrary centred set of integrals, so that $\{Z, \Phi\}=0$ on the whole of $M$. Suppose that it is not necessarily Poisson closed but merely satisfies the condition rank $\{\mathcal{F}, \mathcal{F}\}=l$, where $\mathcal{F}=\left(\mathcal{F}_{1}, \ldots, \mathcal{F}_{l}\right)$. Then the conditions of $Z$-sliding and $\Phi$-sliding specify in $M$ one and the same set $N$. Furthermore, $N$ is $\Phi$-invariant, and if $N$ is smooth, then the system is locally weakly Hamiltonian- $Z$-integrable on $N$.

Actually we consider the more general case where $N$ is specified not in the whole of $M$ but in some submanifold $L_{0} \subseteq M$ and, instead of a centred set $\Phi=(Z, \mathcal{F})$, the set $F$ is somewhat more general.

Proposition 7.3.1. Suppose that we have an arbitrary system with Hamiltonian function $H$. Suppose that there exist two sets of functions $F=\left(F_{1}, \ldots, F_{s}\right)$ and $Z=$ $\left(Z_{1}, \ldots, Z_{k}\right)$ that are regular on the entire phase space $M$ and such that locally $Z=Z(F)$. Let $L_{0} \subseteq M$ be an arbitrary submanifold. Consider the two sets specified in $L_{0}$ by the "sliding" condition with respect to the sets $Z$ and $F$, respectively:

$$
\begin{aligned}
& N_{Z}=\left\{m \in L_{0} \mid d H=\sum_{i=1}^{k} \lambda_{i} d Z_{i} \text { at the point } m\right\}, \\
& N_{F}=\left\{m \in L_{0} \mid d H=\sum_{j=1}^{s} \mu_{j} d F_{j} \text { at the point } m\right\} .
\end{aligned}
$$

Then the following two assertions hold.

A) (Conditions of $Z$ - and $F$-sliding coincide) Suppose that $\left.\{H, F\}\right|_{N_{F}}=0$ and $\left.\{Z, F\}\right|_{N_{F}}=0$. Suppose also that the rank of the Poisson matrix $\{F, F\}$ is constant on $N_{F}$ and

$$
k+\left.(\operatorname{rank}\{F, F\})\right|_{N_{F}}=s .
$$

Then these two sets coincide: $N_{F}=N_{Z}$.

B) (F-invariance of $N_{Z}$, and $Z$-integrability on $\left.N_{Z}\right)$ Suppose that $\{H, F\}=0$ and $\{Z, F\}=0$ and, consequently, $\{Z, Z\}=0$ on the whole of $M$. Suppose in addition that the submanifold $L_{0}$ is invariant under the local phase flows of the systems with Hamiltonians $F_{1}, \ldots, F_{s}$. For example, this always holds for $L_{0}=$ $M$. Then the subset $N_{Z}$ is invariant under the local flows of the same systems and of the original system, that is, the flows of the systems with Hamiltonians $H, F_{1}, \ldots, F_{s}$. If $N$ is a smooth submanifold, then the system is locally weakly Hamiltonian-Z-integrable on $N_{Z}$.

C) (Unifying statement) Suppose that all the conditions of Proposition 7.3.1 B) hold, along with (16). Then both preceding assertions A) and B) hold. In other words, apart from the $(H, F)$-invariance of the subset $N_{Z}$ and apart from the local weakly 
Hamiltonian Z-integrability of the system on $N_{Z}$, in the case where $N_{Z}$ is a submanifold we also have $N_{F}=N_{Z}$.

We emphasize that the coefficients in the formulae for $N_{Z}$ and $N_{F}$ may differ at distinct points $m$, that is, $\lambda_{i}=\lambda_{i}(m), \mu_{j}=\mu_{j}(m)$. We also note that the invariance of any subset under the phase flows of the systems with Hamiltonians $F_{1}, \ldots, F_{s}$ implies its invariance under the flows of the systems with Hamiltonians $Z_{1}, \ldots, Z_{k}$. Under the hypotheses of Proposition $7.3 .1 \mathrm{~B}$ ) to prove that the system is locally weakly Hamiltonian- $Z$-integrable on the submanifold $N_{Z}$ it is sufficient to require simply that it is $Z$-invariant, instead of the $F$-invariance of the submanifold $L_{0}$.

Assertion 7.3.1 C) obviously implies the following.

Corollary 7.3.1 (Integrability on $N_{F}$ when $F$ is closed). Suppose that a system with Hamiltonian function $H$ is defined on a phase space $M$. Suppose that $F=\left(F_{1}, \ldots, F_{s}\right)$ is some set of integrals of the system on $M$ satisfying all the conditions of Proposition 7.2.1, that is, the conditions of regularity, closedness, and the Poisson matrix having constant rank on $M$. Let $\Phi=(Z, \mathcal{F})$ be the centred set equivalent to $F$ described in Proposition 7.2.1, where $Z=\left(Z_{1}, \ldots, Z_{k}\right)$ is its central subset. Suppose that the functions in the set $\Phi$ are defined on the whole of $M$ and that $\Phi=\Phi(F), F=F(\Phi)$, and $\{Z, \Phi\}=0$ also on the whole of $M$.

Then $N_{F}=N_{Z}$, where $N_{F}$ and $N_{Z}$ are the subsets of $M$ specified by the conditions of $F$-sliding and $Z$-sliding, respectively; see formulae (15) and (14). Furthermore, $N=N_{F}=N_{Z}$ is invariant under the phase flows of the systems with Hamiltonians $F_{1}, \ldots, F_{s}$. In addition, if $N$ is a smooth submanifold, then the system on $N$ is locally weakly Hamiltonian-Z-integrable.

The main meaning of Proposition 7.3.1B) is that if the submanifold $L_{0}$ is $Z$-invariant, the system with Hamiltonian $H$ is locally weakly Hamiltonian- $Z$-integrable on $N_{Z}$. Furthermore, $N_{Z}$ is the maximal submanifold of $L_{0}$ having this property. Proposition 7.3.1 C) is useful in the following situation. In concrete cases it is often quite difficult to "isolate" an involutive set $Z$ from the set $F$, using the construction in $\S 7.2$ in the case when $F$ is closed, for example. In the case when (16) holds, so the rank of the Poisson matrix $\{F, F\}$ is maximal, then the submanifold $N_{Z}=N_{F}$ can be specified by the $F$-sliding condition, which here is much more convenient.

However, Proposition 7.3.1 B) shows that the $Z$-sliding condition is more important than the $F$-sliding condition. Indeed, it is the $Z$-sliding condition that is used to specify the maximal $Z$-integrability submanifold in a $Z$-invariant submanifold $L_{0}$ in the most general situation where the system only has an involutive set of integrals $Z$. Proposition 7.3.1 will be proved immediately after the proof of Proposition 7.3.2 stated below, which also demonstrates the fundamental role of the submanifold $N_{Z} \subseteq N_{F}$ in $Z$-integrability.

In particular, in Proposition 7.3.2 we study the case when, apart from $Z$ there is an additional, not necessarily regular set of functions that are common integrals of the systems with Hamiltonian functions in the set $Z$. The existence of such functions allows us, under certain conditions, to obtain certain information on the submanifold $N_{Z}$ and on the existence of common integrals on it, which are essential for the global $Z$-integrability of this system on $N_{Z}$. These conditions imposed on the additional set are, as a whole, weaker than the conditions on the set $F$ in Proposition 7.3.1.

Proposition 7.3.2 (Miscellaneous properties of $N=N_{Z}$ ). Suppose that a system with Hamiltonian function $H$ is defined on some symplectic manifold $M$. Let $Z=\left(Z_{1}, \ldots, Z_{k}\right)$ be an arbitrary regular set of integrals of the system that are pairwise in involution on 
the whole of $M:\{Z, Z\}=0$ and $\{H, Z\}=0$. Let $L_{0} \subseteq M$ be an arbitrary $Z$-invariant submanifold: $J_{m} d Z_{i} \in T_{m} L_{0}, i=1, \ldots, k$, at each point $m \in L_{0}$. Consider the set $N=N_{Z}$ specified in $L_{0}$ by the sliding condition with respect to the set $Z$; see (14). Suppose that $N$ is a smooth submanifold of $M$. Then under these conditions the following four assertions hold.

A) (Z-integrability on $N$ ) The vector fields $J d Z_{1}, \ldots, J d Z_{k}$ and the field $J d H$ are pairwise commutative on the whole of $M$ and are tangent to $N$. Furthermore, the system with Hamiltonian $H$ is locally weakly Hamiltonian-Z-integrable on $N$; see Definition 3.2.4.

B) (Integrals are transferable from $L_{0}$ to $N$ ) Suppose that the fields $J d Z_{1}, \ldots, J d Z_{k}$ have some not necessarily regular set $\mathcal{G}$ of common integrals on $L_{0}$, that is, $d \mathcal{G}(J d Z)=0$ on $L_{0}$. Then the restrictions to $N \subseteq L_{0}$ of the functions in the set $\mathcal{G}$ are common integrals of the systems with Hamiltonians $H, Z_{1}, \ldots, Z_{k}$.

C) (F-invariance of the submanifold $N$ ) Suppose that the same fields $J d Z_{1}, \ldots, J d Z_{k}$ together with the field $J d H$ have some not necessarily regular set $\mathcal{F}$ of common integrals on the whole of $M$, that is, $\{H, \mathcal{F}\}=0$ and $\{Z, \mathcal{F}\}=0$ on $M$. Suppose also that the submanifold $L_{0}$ is $\mathcal{F}$-invariant, that is, invariant under the local phase flow of each system whose Hamiltonian is one of the functions in the set $\mathcal{F}$. Then the submanifold $N$ is $F$-invariant in the same sense, where $F:=(Z, \mathcal{F})$ is the set composed of the sets $Z$ and $\mathcal{F}$.

D) (Toric $Z$-integrability on $N$ ) Suppose that the $k$-dimensional leaves $\Lambda$ of the foliation $\Xi_{N}(J d Z)$ defined by the vector fields in the set $J d Z=\left(J d Z_{1}, \ldots, J d Z_{k}\right)$ on the submanifold $N$ are compact and form a locally trivial fibration of $N$. Then the system is torically weakly Hamiltonian-Z-integrable on $N$; see Definition 3.2.4. In particular, the leaves $\Lambda$ are invariant tori for the system, on which the motion is conditionally periodic. Furthermore, if the restriction $\left.Z\right|_{N}$ is a regular set, then such integrability is in fact stronger -it is Hamiltonian (see the same definition); in particular, the generalization of Gordon's theorem that the frequencies of this motion depend only on the functions in the set $Z$ holds.

Remark 7.3.1. We emphasize that in assertion $\mathrm{C}$ ) it is not required that the set $\mathcal{F}$ be regular anywhere. This means we do not assume that the set $F=(Z, \mathcal{F})$ is closed nor that the Poisson matrix $\{F, F\}$ satisfies any conditions. In assertion B) no conditions at all are imposed on the additional set $\mathcal{G}$ of functions defined on $L_{0}$, except the equality $d \mathcal{G}(J d Z)=0$ on the submanifold $L_{0}$.

It is easy to see that the fact that an arbitrary submanifold is $F$-invariant means more than the vector fields of the systems with Hamiltonian functions only directly involved in the given set $F$ being tangent to it. The formal assertion consists in the following. Add one more function to the set $F$ that is either functionally dependent on the functions in the set $F$ or is equal to the Poisson bracket of some two functions in the set $F_{0}:=F$. The resulting set $F_{1}$ is subjected to the same procedure, the resulting extension of it is denoted by $F_{2}$, and so on. We denote by $\mathcal{P}^{F}$ the uncountable set of all functions on $M$ obtained in this way.

Definition 7.3.1. We call the set $\mathcal{P}^{F}$ the Poisson extension of the set of functions $F$.

Corollary 7.3.2 (Invariance of $N_{Z}$ and $L_{0}$ under the Poisson extension of $F$ ). Suppose that all the conditions of one of Propositions 7.3.1 B), 7.3.1 C), or 7.3.2 C) are satisfied, and let $F$ be the set defined in this proposition. Let $F^{\prime}:=(H, F)$ denote the extension of $F$ by $H$. Then the submanifold $N_{Z}$ specified in $L_{0}$ by (14) is invariant not only under the local phase flows of the systems with Hamiltonian functions directly involved in the set $F^{\prime}$ but also those involved in its Poisson extension $\mathcal{P}^{F^{\prime}}$. Similarly, the submanifold $L_{0}$, which 
is F-invariant by hypothesis, is actually $\mathcal{P}^{F}$-invariant. Corollary 7.3 .1 can be strengthened in the same way: in it $N$ is specified in $L_{0}=M$. Furthermore, $\left\{(H, Z), \mathcal{P}^{F^{\prime}}\right\}=0$ on $M$ in all these cases.

These assertions follow easily from the fact that the operator $J_{m}$ is an isomorphism, using formula (5), which gives the correspondence between Poisson and Lie brackets on a symplectic manifold.

Proof of Proposition 7.3.2. It is more convenient to start by proving assertion C). We have $\{(H, Z),(H, Z, \mathcal{F})\}=0$ on $M$, and $\mathcal{J}_{m} d(Z, \mathcal{F}) \subseteq T_{m} L_{0}$ at all points $m \in L_{0}$. We fix any point $m \in N$ and fix any function $P$ contained in $F=(Z, \mathcal{F})$. Note that the functions in $H, P, Z_{1}, \ldots, Z_{k}$ are pairwise in involution on the whole of $M$. We denote by $Z^{\prime}$ the set that coincides with the set $P, Z_{1}, \ldots, Z_{k}$ if the vector $J_{m} d P$ is not a linear combination of the vectors $J_{m} Z_{1}, \ldots, J_{m} Z_{k}$; otherwise we set $Z^{\prime}=Z$. The vector fields whose Hamiltonians coincide with the functions in $Z^{\prime}$ form a basis $J d Z^{\prime}$ in the abelian Lie algebra $\gamma=\gamma\left(Z^{\prime}\right)$. Consider the orbit $\mathcal{O} \ni m$ of the Lie group corresponding to the algebra $\gamma$. Using local $J d Z^{\prime}$-normalizing coordinates on $\mathcal{O}$ (see Proposition 2.1.1) we can easily show that this group acts locally freely on its orbit $\mathcal{O}$.

We claim that the fields $J d H$ and $J d P$ are tangent to the orbit $\mathcal{O}$ at all of its points. It follows from the construction of $\mathcal{O}$ that the vector $J_{m} d P$ is tangent to $\mathcal{O}$. Since $m \in N=N_{Z}$, the same is also true for the vector $J_{m} d H$. Using the $J d Z^{\prime}$-normalizing coordinates and the pairwise involutivity of the functions in the set $\left(H, Z^{\prime}\right)$ and arguing as in the proof of Proposition 3.4.2 (see $\S 3.4$ ) we find that the fields $J d H$ and $J d P$ are indeed tangent to the orbit $\mathcal{O}$. Moreover, since $J_{x} d H=\sum_{i=1}^{k} \lambda_{i} J_{x} d Z_{i}$ for $x=m$, we see that this relation holds for all $x \in \mathcal{O}$, where the coefficients $\lambda_{i}$ are constant on the entire orbit $\mathcal{O}$. This means that $\mathcal{O} \subseteq N$. Since $\mathcal{O}$ is $P$-invariant, it follows that the trajectory of the field $J d P$ passing through the point $m$ is contained in $\mathcal{O}$ and therefore also in $N$. Consequently, the submanifold $N$ is $F$-invariant, that is, we obtain assertion C).

Now set $F=Z$ on $C$ ), that is, take the empty set for $\mathcal{F}$. Then in this special case, using $\mathrm{C}$ ), the fact that the functions in the set $(H, Z)$ are pairwise commutative and the sliding condition, which obviously holds on the whole of $N$, we obtain that the system with Hamiltonian $H$ is locally weakly Hamiltonian- $Z$-integrable on $N$. Thus, assertion A) is also proved. Assertion B) is obvious. Assertion D) obviously follows from assertion A), Definition 3.2.4, and Theorem 5.5.1 B). The proof of Proposition 7.3.2 is complete.

The proof of Proposition 7.3.1 will be based on the following simple assertion.

Lemma 7.3.1. Let $F=\left(F_{1}, \ldots, F_{s}\right)$ be an arbitrary regular set of functions on a symplectic manifold $M$, and let $m \in M$ be any point of $M$. We set $K_{m}:=\mathcal{J}_{m} d F \cap$ $T_{m} F^{-1}(y)$, where, as before, $\mathcal{J}_{m} d F$ is the linear span of the vectors $J_{m} d Z_{1}, \ldots, J_{m} d Z_{k}$, and $T_{m} F^{-1}(y)$ is the tangent space to the surface $F^{-1}(y)$, where $y=F(m)$. Then the subspace $K_{m}$ is isotropic in $T_{m} M$ and $\operatorname{dim} K_{m}=s-\operatorname{rank}\{F, F\}(m)$. Let $Z=\left(Z_{1}, \ldots, Z_{k}\right)$ be an arbitrary set such that $\mathcal{J}_{m} d Z \subseteq \mathcal{J}_{m} d F$ and $\{Z, F\}(m)=0$. Then $\mathcal{J}_{m} d Z \subseteq K_{m}$.

Proof. First of all we observe that the regularity of the set $F$ implies that the surfaces $F^{-1}(y)$ are smooth submanifolds of codimension $s$ in $M$. Thus, the tangent space $T_{m} F^{-1}(y)$ is well defined. We denote by $\Omega^{2}:=\left.\omega^{2}\right|_{\mathcal{J}_{m}} d F$ the restriction of the symplectic 2 -form $\omega^{2}$ to the subspace $\mathcal{J}_{m} d F$. It easily follows from formula (4) that $\operatorname{rank}\{F, F\}$ at the point $m$ is equal to the rank of the 2 -form $\Omega^{2}$. From this we obtain that the dimension of the kernel of the form $\Omega^{2}$, that is, of the intersection $\mathcal{J}_{m} d F \cap\left(\mathcal{J}_{m} d F\right)^{\perp}$, is equal to $s-\operatorname{rank}\{F, F\}(m)$. Here $\left(\mathcal{J}_{m} d F\right)^{\perp}$ is the skew-orthogonal complement of $\mathcal{J}_{m} d F$ in the tangent space $T_{m} M$. But it follows from Lemma 6.1.2 that $\left(\mathcal{J}_{m} d F\right)^{\perp}=T_{m} F^{-1}(y)$, whence we obtain $\operatorname{dim} K_{m}=s-\operatorname{rank}\{F, F\}(m)$. 
From the condition $\{Z, F\}=0$ and (4) we obtain $d F\left(\mathcal{J}_{m} d Z\right)=0$, that is, $\mathcal{J}_{m} d Z \subseteq$ $T_{m} F^{-1}(z)$. Consequently, $\mathcal{J}_{m} d Z \subseteq \mathcal{J}_{m} d F \cap T_{m} F^{-1}(z)=K_{m}$. The proof of Lemma 7.3.1 is complete.

Proof of Proposition 7.3.1. We begin with assertion A). Since $Z=Z(F)$, we obviously have $\mathcal{J}_{m} d Z \subseteq \mathcal{J}_{m} d F$ at any point $m \in M$. From this we obtain $N_{Z} \subseteq N_{F}$. We now show that $N_{Z} \supseteq N_{F}$. Let $m \in N_{F}$ be an arbitrary point, and $F^{-1}(y) \ni m$ the common level surface of the functions in the set $F$ containing this point. Since $F$ is regular, this surface is smooth. The regularity of $Z$ implies that $\operatorname{dim} \mathcal{J}_{m} d Z=k$. By hypothesis, $\{Z, F\}(m)=0$ and $\operatorname{rank}\{F, F\}(m)=s-k$. Using these facts and the embedding $\mathcal{J}_{m} d Z \subseteq \mathcal{J}_{m} d F$ we obtain from both assertions of Lemma 7.3.1 that

$$
\mathcal{J}_{m} d Z=K_{m}
$$

where, as in the lemma, $K_{m}=\mathcal{J}_{m} d F \cap T_{m} F^{-1}(y)$.

The $F$-sliding condition (see (15)) implies that $J_{m} d H \in \mathcal{J}_{m} d F$. From the equality $\{H, F\}(m)=0$ and (4) we obtain $d F\left(J_{m} d H\right)=0$; hence $J_{m} d H \in T_{m} F^{-1}(y)$. This, with (17) implies that $J_{m} d H \in K_{m}=\mathcal{J}_{m} d Z$. But this means precisely that $m \in N_{Z}$; thus, $N_{Z} \supseteq N_{F}$. Assertion 7.3.1 A) is proved. Assertion 7.3.1 B) is an obvious consequence of Proposition 7.3.2 C). Assertion 7.3.1 C) easily follows from assertions 7.3.1 A) and B). Thus, the proof of Proposition 7.3.1 is complete.

Remark 7.3.2. As usual for toric $Z$-integrability, the condition of the leaves $\Lambda=\Lambda(Z)$ being compact is the strongest and restrictive. This condition is satisfied, for example, in the typical case if the set $Z$ is defined via the Noether duality applied to a global Poisson action of a compact not necessarily abelian Lie group; see $\S 7.1$. The reason is that each leaf $\Lambda \subset N$ of the foliation $\Xi_{N}(J d Z)$ coincides with an orbit of one of the closed abelian subgroups of this group (see Remark 2.3.2); these subgroups may be different for different $\Lambda$. Compact Lie symmetry groups, whose importance is emphasized in this remark, are also important in the Hamiltonian case.

Remark 7.3.3. Another possibility for the global $Z$-integrability of the system on $N$ is the existence on the submanifold $N$ of $r$ functions, including the functions in the set $\left.Z\right|_{N}$, which have the following properties. The differentials of these $r$ functions are linearly independent at each point on $N$, they are all integrals for all $k$ vector fields $J d Z_{1}, \ldots, J d Z_{k}$, and $r=\operatorname{dim} N-k$. (Of course, this is also possible for systems of general form.) Such functions may arise if the set of fields $J d Z=\left(J d Z_{1}, \ldots, J d Z_{k}\right)$ had a set $\mathcal{F}$ of common integrals on $L_{0}$; then by Proposition 7.3.2B) the set $\tilde{\mathcal{F}}:=\left.\mathcal{F}\right|_{N}$ of their restrictions to $N=N_{Z}\left(L_{0}\right)$ consists of common integrals of these fields on $N$. For example, if the set $Z$ is a central subset of an arbitrary centred set $\Phi=(Z, \mathcal{F})$ of functions on the whole of $M$, then the functions in the set $\tilde{\Phi}:=\left.\Phi\right|_{N}$ are common integrals of the fields in the set $J d Z$ on $N$.

When passing from a set of functions $\mathcal{F}$ defined on $L_{0}$ to $\tilde{\mathcal{F}}$, the set of their restrictions to $N$, the number of functionally independent functions among them can decrease. But, first, $\operatorname{dim} N$ usually decreases much more relative to $\operatorname{dim} L_{0}$ in this transition. Second, additional common integrals often emerge on $N=N_{Z}$, which did not exist on $L_{0}$. Thus, it is quite probable that the system is globally weakly Hamiltonian- $Z$-integrable on $N$. If in addition the common level surfaces of all the integrals defined on $N$ are compact, then such integrability is toric.

Definition 7.3.2. Suppose that all the conditions of Proposition 7.3.2 D) hold, except for the condition that the restriction $\left.Z\right|_{N}$ be regular, which is unnecessary. Consider the complete trajectories of the system with Hamiltonian $H$ lying on the submanifold 
$N=N_{Z}$ specified by the $Z$-sliding condition; see (14). Then these trajectories may be called generalized relative equilibrium positions of toric type.

The word "generalized" is used because the "classical" ones (considered, for example, in [3]) correspond to equilibrium positions, that is, to singular points of the reduced system. Recall that if there is a Poisson closed set of integrals $F$, then the reduction is carried out on each common level surface of the integrals of this set by means of factorization by the action of the abelian group defined by the set $Z=Z(F)$. (In 3 , the special case of a set $F$ corresponding to a Poisson action of a Lie symmetry group of the system was considered.) In our terminology, such factorization means that the field of planes $J d Z$ defines a fibration on the surface $F^{-1}(y)$. However, under the hypotheses of Proposition 7.3.2 D) the genuine reduction defined by the flows of the fields $J d Z_{1}, \ldots, J d Z_{k}$ is guaranteed only on the integrability submanifold $N_{Z}$ itself and, generally speaking, it cannot be extended outside $N_{Z}$. Note that due to the condition $\{H, Z\}=0$ imposed on the whole of $M$ such $Z$-reduction of the system nevertheless always exists locally, that is, in a neighbourhood in $M$ of any point $m \in M$.

In the next subsection the condition $\{H, Z\}=0$ will be lifted, and consequently no reduction of this type, not even a local one, will be guaranteed outside $N_{Z}$. Nevertheless, even in this considerably more complicated situation we shall describe a recipe for finding the $Z$-integrability submanifolds $N$. Such submanifolds $N$ may be called "equilibrium submanifolds". The trajectories of the system lying on them are more substantial generalizations of the "classical" relative equilibrium positions.

7.4. Finding the integrability submanifolds of the system in the case where the functions of an involutive set $Z$ are noninvariant. Equilibrium submanifolds. It is clear from the assertions of the preceding subsection that the sliding condition fairly simply and effectively specifies in $M$ the submanifold $N_{Z}$ of local $Z$-integrability of the system; see formula (14), where we set $L_{0}=M$, and Proposition 7.3.2. But here it is assumed that the system is $Z$-invariant, that is, $\{H, Z\}=0$ on the whole of $M$. Of course, this condition is satisfied on any submanifold $N$ of local $Z$-integrability, but it does not have to be satisfied on the whole of $M$. In this subsection we describe a method of finding the $Z$-integrability submanifolds without the assumption that $\{H, Z\}=0$ on the whole of $M$, but this method is more complicated than the method of specifying $N$ by the sliding condition alone.

Recipe for finding the maximal local $Z$-integrability submanifolds $N \subset M$ in the case $\{H, Z\} \neq 0$ and $\{Z, Z\}=0$ on the whole of $M$. This recipe consists in the following. First we impose the sliding condition on the whole of $M$; see formula (14), where we set $L_{0}=M$. We consider this condition more carefully. It is imposed on the points $m \in M$ and requires the differential $d H$ to be linearly dependent on the differentials $d Z_{1}, \ldots, d Z_{k}$ at this point. In arbitrary, not necessarily canonical, coordinates $x=\left(x_{1}, \ldots, x_{2 n}\right)$ this condition can be easily written as a system of the usual equations on $x$ involving the first partial derivatives of the functions $H, Z_{1}, \ldots, Z_{k}$. For example, if $k=1$, that is, $Z=\left(Z_{1}\right)$, then this condition can be written as the following system of $2 n-1$ equations:

$$
\frac{\partial H}{\partial x_{1}} \frac{\partial Z_{1}}{\partial x_{j}}-\frac{\partial H}{\partial x_{j}} \frac{\partial Z_{1}}{\partial x_{1}}=0, \quad j=2,3, \ldots, 2 n,
$$

where it is assumed that $\partial Z_{1} / \partial x_{1} \neq 0$.

In the case of arbitrary $k=1, \ldots, n$ a similar system $G(x)=0$ will consist of $2 n-k$ equations. We assume that the map $Z: M \rightarrow \mathbf{R}^{k}$ has no critical points, that is, the set $Z$ is regular on the whole of $M$. Then such a system specifies the set $L=\Sigma(H, Z)$ 
of critical points of the map $(H, Z): M \rightarrow \mathbf{R}^{k+1}$ defined by the set of $k+1$ functions, $(H, Z)$. We assume that this system of equations $G(x)=0$ can be rewritten in the form $S(x)=0$, where $S$ is some regular set of functions at all the points $m \in L$. Then the level surface $L=S^{-1}(0)$ is a smooth submanifold of $M$.

We can assume that in the typical case the set $G(x)$ of the left-hand sides of all these $2 n-k$ equations of the original system defining $L=G^{-1}(0)$ and generalizing (18) is regular. Then $S=G$ and $\operatorname{dim} L=k$. Since a $Z$-integrability submanifold $N$ must be contained in $L$, the equality $\operatorname{dim} L=k$ means that at best $N$ consists of one leaf $\Lambda=\Lambda(Z)$ of the foliation $\Xi(J d Z)$. But this case is quite degenerate, whereas in the typical case there are no such submanifolds in $M$ at all. Thus, the typical case is just not interesting, and we must seek sets $Z$ such that the critical set $L=\Sigma(H, Z)$ has a higher dimension. Therefore to find the $Z$-integrability submanifolds we cannot choose the involutive set $Z$ arbitrarily, but must choose it from sets connected with the Hamiltonian $H$ of the original system in a certain essential way.

In what follows our goal is to specify the maximal subset $N$ in $L$ that is invariant under the local action of the flows of the systems with Hamiltonians $Z_{1}, \ldots, Z_{k}$. This subset must contain all the submanifolds on which the system is locally weakly Hamiltonian- $Z$ integrable. Actually, the construction of such $N$ is independent of any specific properties of the submanifold $L$ of the symplectic manifold $M$, for example, whether or not the sliding condition holds on $L$. Therefore we describe the construction of $N$ in the general case. In practice, this construction is also independent of the properties of the regular set $Z$, but for simplicity we confine ourselves here to the case of an involutive set.

Construction of the maximal $Z$-invariant subset $N$ contained in a submanifold $L \subset M$ of a symplectic manifold $M$. Thus, we suppose that there is a surface $L \subset M$ defined by a system $S(x)=0$ of $2 n-p$ equations, where $S$ is a set of $2 n-p$ functions defined in some neighbourhood $U$ in $M$ of this surface $L \subset U \subseteq M$. We also assume that $S$ is regular on $L: \operatorname{rank} S_{*}(m)=2 n-p$ at all the points $m \in L$. In particular, this means that $L=S^{-1}(0)$ is an embedded smooth $p$-dimensional submanifold of $M$; see the definition at the beginning of $\S 2.1$. There is also an involutive, regular set $Z$ consisting of $k$ functions on $U:\{Z, Z\}=0$.

To the system $S(x)=0$ ( $S=0$ for short) we add the system $d S(J d Z)=0$, which using the Poisson brackets can also be written in the form $\{S, Z\}=0$. Recall that both $S$ and $Z$ are sets of functions, so that $\{S, Z\}$ is a matrix. For uniformity of notation we rewrite the original system $S=0$ in the form $S_{J d Z}^{(0)}=0$, and the additional one in the form $S_{J d Z}^{(1)}=0$. Then by analogy we add the system $\{\{S, Z\}, Z\}=0$, which we rewrite in the form $S_{J d Z}^{(2)}=0$. Then we successively add the following systems: $S_{J d Z}^{(l)}=0$, $l=3,4,5, \ldots$, and so on to infinity. (This notation is used because for $k=1$ the expression $S_{J d Z}^{(l)}=0$ coincides with the $l$ th derivative of the vector-valued function $S$ along the vector field $J d Z_{1}$.)

We denote by $\hat{N}^{r}$ the subset of $M$ specified by the system

$$
S_{J d Z}^{(l)}=0, \quad l=0,1,2, \ldots, r,
$$

where $r=0,1,2, \ldots, \infty$. It is clear that $\hat{N}^{r} \supseteq \hat{N}^{s}$ for any $r<s \leq \infty$.

Proposition 7.4.1. Under the assumptions made above that the sets of functions $Z$ and $S$ defined on $U$ are regular on the submanifold $L=S^{-1}(0)$, where $\{Z, Z\}=0$ on the whole of $U$, the following two assertions hold.

A) Let $\mathcal{N} \subseteq L$ be any $Z$-invariant submanifold, so that at each point $m \in \mathcal{N}$, $J_{m} d Z_{i} \subseteq T_{m} \mathcal{N}, i=1, \ldots, k$. Then $\mathcal{N} \subseteq \hat{N}^{r}$ for any $r=0,1,2, \ldots, \infty$. 
B) Suppose that the situation is real-analytic. Then $\hat{N}^{\infty}$ is the maximal Z-invariant subset of $L$. Here $Z$-invariance means that if $m \in \hat{N}^{\infty}$, then the leaf $\Lambda_{m}(Z) \ni m$ of the foliation defined by the distribution $\mathcal{J} d Z$ is also contained in $\hat{N}^{\infty}$.

Proof of Proposition 7.4.1. We shall use the following lemma.

Lemma 7.4.1. Suppose that, as in Proposition 7.4.1, the sets of functions $Z$ and $S$ are regular on $L=S^{-1}(0)$ and $\{Z, Z\}=0$ on $U \supset L$, where $U$ is a neighbourhood of $L$ in $M$. Then the set $\hat{N}^{r}, 1 \leq r \leq \infty$, consists of all the points $m \in \hat{N}^{0}$ at which the integral surface $\Lambda_{m}(Z) \ni m$ of the distribution $\mathcal{J} d Z$ has order of tangency to the smooth surface $\hat{N}^{0}=S^{-1}(0)$ greater than $r$. (If these surfaces intersect in general position, then we consider this order to be equal to 1.)

Proof of Lemma 7.4.1. Let $m \in \hat{N}^{0}$ be an arbitrary point and suppose that $\left(y_{1}, \ldots, y_{k}\right.$, $\left.y_{k+1}, \ldots, y_{2 n}\right)$ are local coordinates on $M$ straightening the set of vector fields $J d Z_{1}, \ldots$, $J d Z_{k}$ which are pairwise commutative in a neighbourhood of this point. Recall (see $\S 2.1$ ) that this means that $d y_{i}\left(J d Z_{j}\right)=\delta_{i, j}$ and $d y_{s}\left(J d Z_{j}\right)=0$, where $i, j=1, \ldots, k, s=k+$ $1, \ldots, 2 n$, and $\delta_{i, j}$ is the Kronecker delta. It is easy to see that then for any $l=0,1,2, \ldots$ the set $S_{J d Z}^{(l+1)}$ is obtained from the preceding set $S_{J d Z}^{(l)}$ as follows. It consists of the first partial derivatives of all the functions in the set $S_{J d Z}^{(l)}$ taken with respect to all the first $k$ variables $y_{1}, \ldots, y_{k}$. On the other hand, in these coordinates the leaves $\Lambda$ have the form $\left(y_{k+1}, \ldots, y_{2 n}\right)=$ const. Using the Taylor expansion one can easily obtain the assertion of Lemma 7.4.1 from these facts.

This lemma immediately implies assertion A) of Proposition 7.4.1. Indeed, for each point $m \in \mathcal{N}$ the leaf $\Lambda_{m} \ni m$ is contained in $\mathcal{N}$, and so clearly it is contained in $L \supseteq \mathcal{N}$. Consequently, $m \in \hat{N}^{\infty}$ and therefore $\mathcal{N} \subseteq \hat{N}^{\infty}$. To prove B) we consider any point $m \in \hat{N}^{\infty}$. Then by Lemma 7.4.1 the order of tangency of the surface $L=\hat{N}^{0}$ by the leaf $\Lambda_{m}$ is infinite. This fact and the analyticity of the situation imply that $\Lambda_{m} \subseteq L$. From this embedding and again from Lemma 7.4.1 we obtain that $\Lambda_{m} \subseteq \hat{N}^{\infty}$ and therefore $\hat{N}^{\infty}$ is indeed $Z$-invariant. The proof of Proposition 7.4.1 is complete.

Definition 7.4.1. We call the system of equations (19) the tangency condition of tangency with order greater than $r$ between the submanifold $L=\hat{N}^{0}$ and the leaves $\Lambda=\Lambda(Z)$ defined by the distribution $\mathcal{J} d Z$, where $r=0,1,2, \ldots$ For $r=\infty$ the order of tangency is considered to be infinite.

In the typical case one can expect that if there exist $Z$-invariant submanifolds $\mathcal{N} \subseteq$ $L$, then the " $r$-shortened" system (19) will soon stabilize as $r$ increases, specifying in the process the surface $N_{\mathrm{st}}=\bigcup \mathcal{N}$ consisting entirely of such submanifolds $\mathcal{N}$, whose dimension must be at least $k$. However, if there does not exist any such $Z$-integrability submanifold $\mathcal{N}$, then the shortened system, as $r$ increases, will either very soon become contradictory or will begin to specify only those surfaces $\hat{N}^{r}$ whose dimension is less than $k$. This is what would indicate the nonexistence of $Z$-invariant submanifolds in $L$.

The number of equations in the subsystem $S_{J d Z}^{(l)}=0$ is equal to $k^{l}(2 n-p)$, that is, it is usually relatively large, and for $k \geq 2$ this number can even increase in a geometric progression depending on $l$. In any case the total number of equations in system (19) rapidly increases as $r$ increases. Therefore, in all probability, in real examples of systems with not too many degrees of freedom it will be, as a rule, sufficient to study the $r$ shortening (19) for $r=1$ or at most $r=2$. This is sufficient either to prove the nonexistence of such surfaces $\mathcal{N}$ or to obtain a system of equations defining the union of all the $Z$-invariant surfaces contained in $L$. 
Remark 7.4.0. It is easy to verify that all the assertions above in this subsections remain valid if, instead of an involutive set $Z$, we consider an arbitrary Poisson closed regular set $F$. Using the recipe given above one can seek the maximal $F$-invariant subsets $\mathcal{N} \subseteq L$ even for an arbitrary regular set $F$, since at least Proposition 7.4.1 A) remains valid. The above arguments on the quick stabilization of $\hat{N}^{r}$ as $r$ increases also do not change for such a set.

We now return to the situation considered at the beginning of this subsection where $L=S^{-1}(0)$ is specified in $M$ by the $Z$-sliding condition (14). Then Proposition 7.4.1, Definition 3.2.4, Corollary 3.4.3, and Theorem 5.5.1 obviously imply the following.

Corollary 7.4.1. Suppose that $L=S^{-1}(0)$ is specified by condition (14) where $L_{0}=M$. In other words, in the notation of equality (14) for $L$ we take $L=N_{Z}$ with $L_{0}=M$. Suppose that, as in Proposition 7.4.1, the sets of functions $Z$ and $S$ are regular on $L$ and are now taken to be defined on the whole of $M$, and let $\{Z, Z\}=0$ on the whole of $M$. Then the following two assertions hold.

A) Let $\mathcal{N} \subseteq M$ be any submanifold on which the system is locally $Z$-integrable, that is, it has a set of sliding weakly Hamiltonian symmetries $Z$ on $\mathcal{N}$. Then $\mathcal{N} \subseteq \hat{N}^{r}$ for any $r=0,1,2, \ldots, \infty$, where $\hat{N}^{r}$ is specified in $L$ by the condition of tangency of order greater than $r$ of $L$ by the leaves $\Lambda=\Lambda(Z)$, given by formula (19).

B) Suppose that the situation is real-analytic. Suppose that some connected component $N$ of the subset $\hat{N}^{\infty}$ is a submanifold of $M$. Suppose also that the restriction $\left.Z\right|_{N}$ of the set $Z$ to $N$ is regular. Then the system is locally strongly Hamiltonian-Z-integrable on $N$; see Definition 3.2.4. In particular, if the leaves $\Lambda=\Lambda(Z)$ defined by the vector fields $J d Z_{1}, \ldots, J d Z_{k}$ are compact and form a locally trivial fibration of $N$, then the generalizations of Gordon's theorem stated in Theorem 5.5.1 A) and B) hold.

This assertion remains valid if, instead of a connected component of the subset $\hat{N}^{\infty}$, we take for $N$ any $Z$-invariant submanifold contained in $\hat{N}^{\infty}$; moreover, in this case it is not necessary to require the situation to be analytic.

Suppose that, as in Corollary 7.4.1, $\hat{N}^{\infty}$ is specified by condition (19) in the submanifold $L=S^{-1}(0)=\hat{N}^{0}$, which in turn is specified in the whole of $M$ by the $Z$-sliding condition (14) with $L_{0}=M$. Let $N \subseteq \hat{N}^{\infty}$ be an arbitrary maximal submanifold, that is, it cannot be extended further, on which the system has the set of sliding weakly Hamiltonian symmetries $Z$. The dimension of $N$ can be arbitrary, including the minimum possible, that is, $k$.

Definition 7.4.2. In this case we call $\hat{N}^{\infty}$ the complete $Z$-equilibrium subset of the system. The submanifold $N$ is called a $Z$-equilibrium submanifold, as well as a submanifold of locally weakly Hamiltonian integrability of critical type. If in addition the system is torically $Z$-integrable on $N$, then we replace "locally weakly Hamiltonian" by "weakly Hamiltonian-toric".

Remark 7.4.1. The term "critical" is used here for the following reasons. First, $L$ is a set of critical points of the map $(H, Z): M \rightarrow \mathbf{R}^{k+1}$; see condition (14) with $L_{0}=M$. Second, each subsystem $S_{J d Z}^{(l)}=0$ contained in system (19) specifying $\hat{N}^{r}$ in $L$ also defines the critical set of points where the vector fields $J d Z_{1}, \ldots, J d Z_{k}$ are tangent to the surface (19) defined earlier, with $r=l-1$, that is, $\hat{N}^{l-1}$.

Somewhat differently and completely in geometric language, all these conditions of criticality can be interpreted as conditions of tangency to the leaves $\Lambda=\Lambda(Z)$ corresponding to the distribution $\mathcal{J} d Z$. Indeed, the sliding condition (see (14) for $L_{0}=M$ ) 
specifies in $M$ the subset $L$ of all the points $m \in M$ at which the vectors of the original field $J d H$ are exactly tangent to the leaves $\Lambda$. The role of the tangency conditions (19) is that they specify in $L$ the tangency points of order $>r$ of the manifold $L$ by the same leaves $\Lambda=\Lambda(Z)$.

Remark 7.4.2. We emphasize that for an equilibrium submanifold no reduction defined by the fields $J d Z_{1}, \ldots, J d Z_{k}$ is required, even locally; cf. the remark after Definition 7.3.2. Indeed, the field $J d H$ of the system does not have to commute with the fields $J d Z_{1}, \ldots, J d Z_{k}$ outside $\hat{N}^{\infty}$, and a fortiori not on the whole of $M$.

Remark 7.4.3. In our context the facts given in the last two subsections can be interpreted as follows. In the end, to find the submanifolds of global Hamiltonian integrability it is important to have a set $Z$ of functions in involution: $\{Z, Z\}=0$. Then the submanifold $N$ of local $Z$-integrability is specified. It is specified either by the sliding condition alone - in the case where the system is $Z$-invariant, or by the sliding condition and the $Z$-tangency condition in Definition 7.4.1 and then verifying that $\left.Z\right|_{N}$ is regular. The next important point is to verify the condition that the leaves $\Lambda$ form a fibration of $N$. Thus, it is not necessary that the functions in the set $Z$ be integrals of the system on the whole of $M$, as was required in $\S 7.3$. The existence of an arbitrary Lie group $G$ with Poisson action or of a Poisson closed set of functions $F$ on $M$ can ensure the existence of such a set $Z$ and can provide the leaves $\Lambda=\Lambda(Z)$ with the required properties.

Remark 7.4.4. Practically the same formulae, as written down above (see (14) and (19)), can also be used to specify the integrability submanifolds of systems of general form, that is, ones that are not necessarily Hamiltonian. More precisely, let $A=\left(a_{1}, \ldots, a_{k}\right)$ be a basis of some abelian Lie algebra acting in the phase space $M$ of a vector field $V$. Then we must write down the sliding condition (1) in the form of a system of equations specifying an analogue of the submanifold $L$. Then we must "repeatedly" subject the left-hand sides of these equations to the action of the linear operators defined by the fields $a_{1}, \ldots, a_{k}$ equating the results of the action to zero. In the typical case we obtain an $A$-invariant subset, which must contain all the submanifolds of local Liouville $A$-integrability of the field $V$; see Definition 2.1.3.

But it is not possible to specify the submanifolds of local $A$-integrability of the field $V$ in this way immediately, by verifying some simple condition such as the regularity of the set $\left.Z\right|_{N}$, as was the case in the strongly Hamiltonian situation. In addition we must impose the condition $[V, A]=0$, that the Poisson bracket of the field $V$ with the fields in the set $A$ vanishes; more precisely, we must use this condition to specify a smaller submanifold in the submanifold just obtained. Then we must again specify an $A$-invariant submanifold in it. The specifying of submanifolds can be carried out in the following order: by the sliding conditions, by the condition of infinitesimal commuting $[V, A]=0$, and then by the condition of invariance.

Incidentally, using this method, that is, using the condition $[J d H, J d Z]=0$, one can specify the submanifolds of local weakly Hamiltonian $Z$-integrability of the field $J d H$ in the Hamiltonian situation if $\left.Z\right|_{N}$ is not regular. More precisely, suppose that, acting according to the recipe of Corollary 7.4.1, we obtain a $Z$-invariant submanifold $N \subseteq \hat{N}^{\infty}$ satisfying the $Z$-sliding condition but not satisfying the condition $\operatorname{rank}\left(\left.Z\right|_{N}\right)_{*}=\operatorname{rank} Z_{*}$, that $Z$ is internally regular on $N$. We specify a subset in $N$ by two conditions: first by the condition $[J d H, J d Z]=0$, and then by the condition of $Z$-invariance. Then the subset obtained is a $Z$-equilibrium subset, and any $Z$-invariant submanifold $N^{\prime}$ contained in such a subset is locally weakly Hamiltonian- $Z$-integrable; see Definition 3.2.4. Using this fact one can easily obtain an analogue of Corollary 7.4.1 for weakly Hamiltonian $Z$-integrability. 
7.5. General remarks on methods for finding the integrability submanifolds of the system, and the mechanisms of such integrability. The algorithms for specifying the submanifolds $N$ of local $Z$-integrability considered in $\S \S 7.3$ and 7.4 will by no means always solve the problems, since it is global integrability that gives the solutions a simple structure and has real value for applications; see also Remark 3.2.6. This problem is solved if the set $Z$ is constructed from the set of functions $F$ corresponding to a Poisson action of a compact Lie group on $M$ using the scheme in $\S 7.1$; cf. Remark 2.3.2. Another possibility is that sufficiently many integrals, common to the flows of the systems with Hamiltonians $Z_{1}, \ldots, Z_{k}$ that form the set $Z$, exist on $N$. But what should be done in the case where the number $r$ of functionally independent integrals is not large enough for global integrability, that is, when $r+k<\operatorname{dim} N$ ? This concerns both the case where $\{H, Z\}=0$ on the whole of $M$ considered in $\S 7.3$ and the case of arbitrary $\{H, Z\}$ studied in $\S 7.4$.

Moreover, it is often necessary to include the Hamiltonian $H$ itself into the set of functions $Z$. But then neither the sliding condition, nor the tangency condition, nor the condition of infinitesimal commuting $[J d H, J d Z]=0$ can specify anything in $M$, since they hold on the whole of $M$ automatically, so that by specifying a submanifold we would obtain $N=M$. The system is weakly integrable on the whole of $M$; but if there is no global integrability, then we cannot obtain it using these conditions. In this case the considerations related to the compactness of a Lie group do not save the day either. Indeed, if the set $Z$ with $H$ included defines a Lie algebra of a Lie group, then it is only very rarely compact. Even if a compact Lie group corresponded to this set before $H$ was added to the set $Z$, then after including $H$ the compactness is almost always lost.

In order to get out of this stalemate we can consider some invariant submanifold $L \subset N$ for the system and then apply the algorithm of $\S 7.4$ to find a $Z$-invariant subset of $L$. We assume that the two other necessary conditions for local $Z$-integrability, the sliding condition and the condition $[J d H, J d Z]=0$, are satisfied on $N$; then they are also satisfied on any submanifold, so that they no longer have to be verified on $L$. But finding proper invariant submanifolds in an invariant manifold is often quite a difficult problem; therefore we can act as follows. We can specify in some way a submanifold in $N$ that is not necessarily invariant for the system but presumably contains a submanifold of global $Z$-integrability. Then using the same $Z$-tangency condition (19) we can specify $Z$-integrability submanifolds $N_{1} \subset L \subset N$, which in fact are not unique.

If the integrability of the system on some of these submanifolds $N_{1}$ is again merely local, then all the actions should be repeated, starting from the choice of the submanifold $L_{1}$ in $N_{1}$, and again this choice is made based on certain "external" considerations. Thus, an outline of the following scheme appears.

"Extractive" scheme for finding the global $Z$-integrability submanifolds. Let $Z=\left(Z_{1}, \ldots, Z_{k}\right)$ be a regular set of functions involutive on $M,\{Z, Z\}=0$. Then finding such submanifolds can be represented as the construction of a chain of embedded submanifolds

$$
M=N_{0} \supset N_{1} \supset N_{2} \supset \cdots \supset N_{p},
$$

whose dimensions are strictly decreasing, so that $p \leq 2 n-k$. Each passage $N_{j} \rightarrow N_{j+1}$ is performed on the basis of the following four considerations.

a) (Sliding) $N_{j+1}$ is specified in $N_{j}$ by the sliding condition

$$
d H=\sum_{i=1}^{k} \lambda_{i} d Z_{i},
$$

that is, by condition (14) in which we put $L_{0}=N_{j}$. 
b) (Tangency or invariance) $N_{j+1}$ is specified in $N_{j}$ by the condition of $Z$-invariance $J_{m} d Z_{i} \in T_{m} N_{j+1}, i=1, \ldots, k, m \in N_{j+1}$, which was studied in $\S 7.4$; by the condition of $H$-invariance $J_{m} d H \in T_{m} N_{j+1}, m \in N_{j+1}$, that is, by specifying the maximal invariant submanifold for the system in $N_{j}$; or by alternating these two conditions; or by at once imposing the condition of $(H, Z)$-invariance, where $(H, Z)$ is the extension of the set $Z$ by $H$.

c) (Infinitesimal commuting) $N_{j+1}$ is specified in $N_{j}$ by the condition

$$
[J d H, J d Z](m)=0, \quad m \in N_{j} .
$$

d) (Other methods of specifying a submanifold) $N_{j+1}$ is specified in $N_{j}$ on the basis of other considerations related to the specifics of the system, the set $Z$, and the submanifold $N_{j}$.

As for item b) concerning specifying a submanifold by the tangency conditions, which of the possibilities listed there should be chosen in practice is determined by how convenient it is to apply it to a concrete problem. It seems that in many cases, especially if it is required to obtain toric integrability, it is sufficient to specify the submanifold $N_{j+1}$ in $N_{j}$ simply by the condition of $H$-invariance.

By the words "is specified by the condition..." here we do not mean specifying the submanifold strictly, as in $\S \S 7.3$ and 7.4, but one based on this specification. For example, a strictly specified subset may not be smooth and at least must be divided into smooth strata. We can assume that at the end of such a chain we obtain either the empty set or a submanifold $N=N_{p}$ of local weakly Hamiltonian $Z$-integrability. Suppose that we succeed in obtaining an $N$, on which there are $r=\operatorname{dim} N-k$ functionally independent integrals common for the flows of all the fields $J d Z_{i}, i=1, \ldots, k$. Then the $Z$-integrability of the system on $N$ will be the required global one.

Note that if the sliding condition a) and the commutativity condition c) are satisfied at some step, then they do not have to be verified further, since they will be satisfied automatically. Thus, one can use them in the construction of the chain (20) only once each. Note also that if the submanifold $N_{j}$ obtained after the $j$ th step of the construction of the chain satisfies the sliding condition, the $Z$-invariance condition and the regularity condition, $\operatorname{rank}\left(\left.Z\right|_{N_{j}}\right)_{*}=k$, then the commutativity condition c) will be necessarily satisfied for this $N_{j}$, so that for $j^{\prime}>j$ it no longer needs to be verified. But the condition of internal regularity for the set $Z$ can actually be violated as $j$ increases. Therefore after obtaining the last submanifold $N=N_{p}$ of the chain one has to verify this condition again in order to find out whether $N$ is a submanifold of strongly Hamiltonian $Z$-integrability.

The first three methods a), b), and c) of specifying $N_{j+1}$ in $N_{j}$ can be formalized to a sufficient extent, and the first two of them were described in detail in $\S \S 7.3$ and 7.4. No special conditions are assumed for the order of the methods a), b), c), and d) in the construction of the chain (20); for example, a "free" choice of d) might be the start.

Remark 7.5.1. The situation for the common integrals of the vector fields $J d Z_{1}, \ldots, J d Z_{k}$ that are additional to the set $Z$ is completely analogous to that considered in $\S 7.3$. There, when $\{H, Z\}=0$ a $Z$-integrability submanifold is specified by the sliding condition; see Remark 7.3.3. Namely, looking only at those submanifolds $N_{j}$ in the resulting chain (20) that are $Z$-invariant, we may trace two tendencies as $j$ increases. All such integrals, including the set $Z$, are conserved as $j$ increases, so that formally their number can increase since new integrals may appear as the dimension of the surfaces $N_{j}$ decreases. But the number $r$ of functionally independent ones among them can still decrease because the "earlier" integrals are restricted to submanifolds of smaller dimension. Nevertheless, in concrete situations one can hope that the " $Z$-integrability deficiency" $\operatorname{dim} N_{j}-k-r$ will be rapidly decreasing as $j$ increases, due to a rapid decrease of $\operatorname{dim} N_{j}$. 
Remark 7.5.2. An interesting question is on which basis is the submanifold $N_{j+1}$ chosen in $N_{j}$ when the "free" method d) is realized? It is possible that the reasons for the choice of $N_{j+1} \subset N_{j}$ are diverse and are determined by a concrete situation. As an example we give the considerations on which the choice of $N_{1} \subset N_{0}=M$ is made, to find periodic solutions in the $s$-body problem using the so-called "central configurations"; see, for example, 25, 26]. (These solutions were mentioned at the end of $\S 1$.)

A central configuration is an arrangement of the bodies of the system such that the resultant force $f_{i}$ acting on each body due to the other $s-1$ bodies is directed towards the centre of mass $O$, and the ratio of the quantity $\left|f_{i}\right|$ to $m_{i} \rho_{i}$ is independent of the body, that is, of $i:\left|f_{i}\right| /\left(m_{i} \rho_{i}\right)=$ const (but it may depend on time); here $i=1, \ldots, s$ is the number of a body, $\rho_{i}$ is the distance from the $i$ th body to the centre of mass $O$, and $m_{i}$ is the mass of this body. In other words, the ratio of the magnitude of the acceleration of the body to its distance from the centre of mass $O$ is independent of the body.

It is easy to see that in the canonical coordinates in $M \subset \mathbf{E}^{6(s-1)}$ induced by Cartesian coordinates in the "physical" space $\mathbf{E}^{3}$ these conditions are given by algebraic equations, which can be relatively easily studied. They specify a set of separate submanifolds, and possibly also of families of submanifolds in the configuration space and therefore also in the phase space $M=M^{6(s-1)}$, such that each of these submanifolds corresponds to its own arrangement of the bodies in the space $\mathbf{E}^{3}$ up to similarity. We fix any of these submanifolds $N_{1} \subset M$ corresponding to a planar arrangement of the bodies. It is easy to show that this submanifold contains closed trajectories, which form a submanifold of toric $H$-integrability of the system, which we denote by $N_{2}$. (This analysis in a way replaces the process of specifying an $H$-invariant submanifold in $N_{1}$, mentioned above, which is a somewhat more cumbersome operation.)

The motion of the bodies corresponding to the trajectories lying in such $N=N_{2}$ is planar, that is, all the bodies of the system move in the same plane. If we fix such a plane, then it is easy to show that to each "planar" central configuration there corresponds a 3parameter family of "Kepler-like" periodic trajectories. When we again pass to 3-dimensional space, the number of parameters increases to 5 . The number of configurations with planar motion corresponding to fixed $s$ increases rapidly as $s$ increases.

The submanifold $N=N_{2}$ itself is a 6-dimensional symplectic submanifold. As in $\S 1$, let $H$ and $\overrightarrow{\mathcal{M}}$ be the total energy and vector of angular momentum respectively, taken over all the bodies of the system. Then on such a submanifold $N \subset M$ the system is globally Hamiltonian- $Z$-integrable, where $Z=\left(Z_{1}\right)$ and $Z_{1}=H$. For $H<0$ and $\overrightarrow{\mathcal{M}} \neq 0$ the $Z$-integrability on $N$ is toric, that is, under these restrictions all the solutions of the system with initial conditions on $N$ are periodic. These solutions satisfy all the generalizations of Gordon's theorem, that is, the period and action depend only on the total energy $H$. Apart from Theorems 5.5.1 A) and B) this also follows from the fact that all the solutions with trajectories on this $N$ are "Kepler-like". By this we mean that the restriction to the whole of $N$ of the system under consideration with $3(s-1)$ degrees of freedom, $s \geq 3$, is completely isomorphic to the integrable system corresponding to the two-body problem, that is, to the case $s=2$.

Note that since we are studying $H$-integrability, there is no sense in using the $H$-sliding condition to specify a submanifold. We can take $\mathcal{M}=|\overrightarrow{\mathcal{M}}|$ for $Z_{1}$ instead of $H$; then the $\mathcal{M}$-sliding condition specifies in the whole of $M$ the submanifold corresponding to the planar motion of the bodies obtained by a rotation of $\mathbf{E}^{3}$ together with the "frozen-in" bodies preserving this plane with constant angular velocity around the centre of mass. (An example is provided by the motion corresponding to the Lagrange periodic solutions; see $\S 1.1$.) But such circular motions are special cases of the motions corresponding to central configurations. The corresponding trajectories in $M$ are relative equilibrium 
positions (cf. Definition 7.3.2), since the phase flow of the system with Hamiltonian function $\mathcal{M}$ is periodic and therefore defines a factorization of $M$.

This $\mathcal{M}$-factorization can be used to find submanifolds of periodic trajectories in the reduced system obtained by the factorization. Such submanifolds can be lifted to submanifolds of toric $\mathcal{M}$-integrability fibred into the 2 -dimensional invariant tori - submanifolds of "relatively closed trajectories". Here the arrangements of the moving bodies, generally speaking, will no longer form central configurations. There are many studies devoted to this approach; see, for example, [22].

Remark 7.5.3. The analysis in $\S 7$ shows that finding the $Z$-integrability submanifolds $N$ by successive specification by systems of equations defining either the $Z$-sliding condition, or the condition of $Z$-invariance, or the condition of $Z$-commuting, or some other conditions of similar type is a fairly simple and effective method. All these conditions are characterized by the fact that they specify a submanifold itself, rather than, say, its intersection with some transversal like the Poincaré section.

But this "critical" approach is far from being the only one. This is, in particular, indicated by Example 1 in $\S 1.1$, in which none of these three conditions $-Z$-sliding, $Z$-invariance and of $Z$-commuting — works in principle, since they are automatically satisfied on the whole of $M$. The reason is again in the fact that the Hamiltonian $H$ is contained in the set $Z$ : in this example $k=1$, that is, $Z=\left\{Z_{1}\right\}$, and $Z_{1}=H$. Nevertheless, submanifolds on which the system is integrable do exist. Nor does it seem possible to specify them using any systems of equations according to the "forcible" method d). Here one must rather apply methods such as considering a Poincaré section and the Poincaré return map on it, and other similar methods used for finding periodic solutions; see, for example, 7, 6, 28, 29, 30, 31, 22.

Actually, toric $Z$-integrability on submanifolds is related to the fact that, generally speaking, noncompact leaves $\Lambda=\Lambda(Z)$ defined by the vector fields $J d Z_{1}, \ldots, J d Z_{k}$, where $Z=\left(Z_{1}, \ldots, Z_{k}\right)$ is a set of functions in involution, can sometimes "roll up" into compact surfaces, which must be tori. The leaves $\Lambda(Z)$ roll up into tori in about the same way as the trajectories of a vector field become closed, which is a special case of the general situation for $k=1$. Suppose that the sliding conditions are satisfied on some of these tori $\Lambda$. Then they are often invariant isotropic tori of the system such that the motion on them is conditionally periodic; for example, this is always the case if $H=Z_{1}$ or if $\{H, Z\}=0$ in a more general case. Usually this happens with entire families of leaves $\Lambda$, which is what causes the appearance of $Z$-integrability submanifolds $N=\bigcup \Lambda$. But the reasons for such "rolling up" of the leaves $\Lambda=\Lambda(Z)$, as well as for the appearance of closed trajectories, can be different: from reasons of "critical type", to "random" ones; see, for example, [7, 28, 30, 31]. In principle, all this of course relates also to systems of general form.

Remark 7.5.4. We emphasize that in $\S \S 3-5$ we mainly considered essentially weaker conditions on the set $Z$ and submanifold $N$ than in $\S 7$. In particular, the condition $\{H, Z\}=0$, that is, that $Z$ is a set of integrals of the system, was almost completely redundant, and only the sliding condition on $N$ was used. In addition, we assumed that the functions in the set $Z$ were pairwise in involution also only on the submanifold $N \subset M$, rather than on the whole of $M$ as in this $\S 7$. The reason is that in $\S \S 3-5$ a $Z$-integrability submanifold $N$, with rare exceptions, was considered to be given; in particular, we were assuming that the vector fields $J d Z_{1}, \ldots, J d Z_{k}$ were tangent to $N$, while in $\S \S 7.3-7.5$ we were seeking such submanifolds. Of course, not only the condition $\{H, Z\}=0$, but also the condition $\{Z, Z\}=0$ required in $\S 7$ in the whole of $M$ can be weakened when finding the integrability submanifolds in the general case. 


\section{REFERENCES}

[1] V. I. Arnol'd, Mathematical methods of classical mechanics, 3rd ed., Nauka, Moscow, 1989; English transl. of 2nd ed., Springer-Verlag, New York, 1989. MR1037020 (93c:70001)

[2] V. I. Arnold, V. V. Kozlov, and A. I. Neishtadt,Mathematical aspects of classical and celestial mechanics, URSS, Moscow, 2002; English transl. of the 1985 Russian original, Springer-Verlag, Berlin, 1997. MR 1656199 (2000b:37054)

[3] V. I. Arnol'd and A. B. Givental', Symplectic geometry, VINITI, Moscow, 1985; English transl., Springer-Verlag, Berlin, 2001. MR0842908 (88b:58044)

[4] N. N. Nekhoroshev, Two theorems on action-angle variables, Uspekhi Mat. Nauk 24 (1969), no. 5, 237-238. (Russian) MR0261824 (41:6435)

[5] N. N. Nekhoroshev, Action-angle variables and their generalizations, Trudy Moscow Math. Soc. 26 (1972), 181-198. (Russian) MR0365629 (51:1881)

[6] N. N. Nekhoroshev, Families of invariant isotropic tori of Hamiltonian systems, Uspekhi Mat. Nauk 40 (1985), no. 5, 217. (Russian)

[7] N. N. Nekhoroshev, The Poincaré-Lyapunov-Liouville-Arnol'd theorem, Funktsional. Anal. i Prilozhen. 28 (1994), no. 2, 67-69; English transl. in Funct. Anal. Appl. 28 (1994), 128-129. MR 1283258 (95e:58072)

[8] N. N. Nekhoroshev, Generalizations of Gordon's theorem, Proc. Symmetry and perturbation theory (Cala Gonone, Sardinia, Italy, 2001), World Sci. Publ., River Edge, NJ, 2001, pp. 137-142. MR:1875487 (2002g:37002)

[9] N. N. Nekhoroshev, D. A. Sadovskiǔ, and B. I. Zhilinskii, Fractional monodromy of resonant classical and quantum oscillators, C. R. Math. Acad. Sci. Paris 335 (2002), 985-988. MR2030223 (2004j:37143)

[10] N. N. Nekhoroshev, Generalizations of Gordon theorem, Regul. Chaotic Dyn. 7 (2002), 239-247. MR 1931395 (2003j:37082)

[11] V. Guillemin and S. Sternberg, Geometric asymptotics, Amer. Math. Soc., Providence, RI, 1977. MR 0523209 (58:25636)

[12] W. Gordon, On the relation between period and energy in periodic dynamical systems, J. Math. Mech. 19 (1969/1970), 111-114. MR0245930(39:7236)

[13] V.V. Kozlov, Symmetries, topology and resonances in Hamiltonian mechanics, Udmurt Univ., Izhevsk, 1995; English transl., Springer-Verlag, Berlin, 1996. MR1443434 (97j:70010b)

[14] M. V. Karasëv and V.P. Maslov, Nonlinear Poisson brackets. Geometry and quantization, Nauka, Moscow, 1991; English transl., Amer. Math. Soc., Providence, RI, 1993. MR1214142 (94a:58072)

[15] A.S. Mishchenko and A.T. Fomenko, Generalized Liouville method of integration of Hamiltonian systems, Funkcional. Anal. i Prilozhen. 12 (1978), no. 2, 46-56; English transl. in Funct. Anal. Appl. 12 (1978), 113-121. MR0516342 (58:24357)

[16] A. T. Fomenko, Symplectic geometry, Moscow Univ., Moscow, 1988; English transl., Gordon and Breach, Luxembourg, 1995. MR 1673400 (99k:58068)

[17] A.D. Bruno, The restricted 3-body problem: plane periodic orbits, Nauka, Moscow, 1990; English transl., Walter de Gruyter, Berlin, 1994. MR1301328 (95g:70007)

[18] G. N. Duboshin, Celestial mechanics. Analytic and qualitative methods, Nauka, Moscow, 1978. (Russian)

[19] R. L. Bishop and R. J. Crittenden, Geometry of manifolds, AMS Chelsea Publishing, Providence, RI, 2001. MR 1852066 (2002d:53001)

[20] A. V. Borisov and I. S. Mamaev, Poisson structures and Lie algebras in Hamiltonian mechanics, Udmurt. Univ., Izhevsk, 1999. (Russian) MR:1707317 (2000k:37073)

[21] J. Moser, Regularization of Kepler's problem and the averaging method on a manifold, Commun. Pure Appl. Math. 23 (1970), 609-636. MR0269931 (42:4824)

[22] E. A. Kudryavtseva, Generalization of geometric Poincaré theorem for small perturbations, Regul. Chaotic Dyn. 3 (1998), no. 2, 46-66. MR1693474 (2000d:37072)

[23] J. Marsden and A. Weinstein, Reduction of symplectic manifolds with symmetry, Rep. Math. Phys. 5 (1974), no. 1, 121-130. MR 0402819 (53:6633)

[24] G. V. Gorr, L.V. Kudryashova, and L. A. Stepanova, Classical problems in the theory of solid bodies. Their development and current state, Naukova Dumka, Kiev, 1978. (Russian) MR0519067 (80m:70001)

[25] F. Pacella, Central configurations of the $N$-body problem via equivariant Morse theory, Arch. Rational Mech. Anal. 97 (1987), no. 1, 59-74. MR0856309 (87k:70014)

[26] A. Chenciner and A. Venturelli, Minima de l'intégrale d'action du problème newtonien de 4 corps de masses égales dans $\mathbf{R}^{3}$ : orbites "hip-hop", Celestial Mech. Dynam. Astronom. 77 (2000), no. 2, 139-152. MR.1820355(2001k:70012) 
[27] A. Albouy and A. Chenciner, Le problème des $n$ corps et les distances mutuelles, Invent. Math. 131 (1998), 151-184. MR.1489897 (98m:70017)

[28] D. Bambusi and G. Gaeta, On persistence of invariant tori and a theorem by Nekhoroshev, Math. Phys. Electron. J. 8 (2002), Paper 1, 13 pp. (electronic). MR.1922423 (2003f:37097)

[29] D. Bambusi and D. Vella, Quasi periodic breathers in Hamiltonian lattices with symmetries, Discrete Contin. Dyn. Syst. Ser. B 2 (2002), 389-399. MR1898321 (2003b:37117)

[30] G. Gaeta, The Poincaré-Lyapounov-Nekhoroshev theorem, Ann. Physics 297 (2002), no. 1, 157173. MR.1900063 (2003b:37085)

[31] G. Gaeta, The Poincare-Nekhoroshev map, J. Nonlinear Math. Phys. 10 (2003), no. 1, 51-64. MR $1943943(2003 \mathrm{j}: 37107)$

Lomonosov Moscow State University, Leninskie Gory, Moscow, GSP-2, 119992, Russia

E-mail address: nekhoros@mech.math.msu.su 